
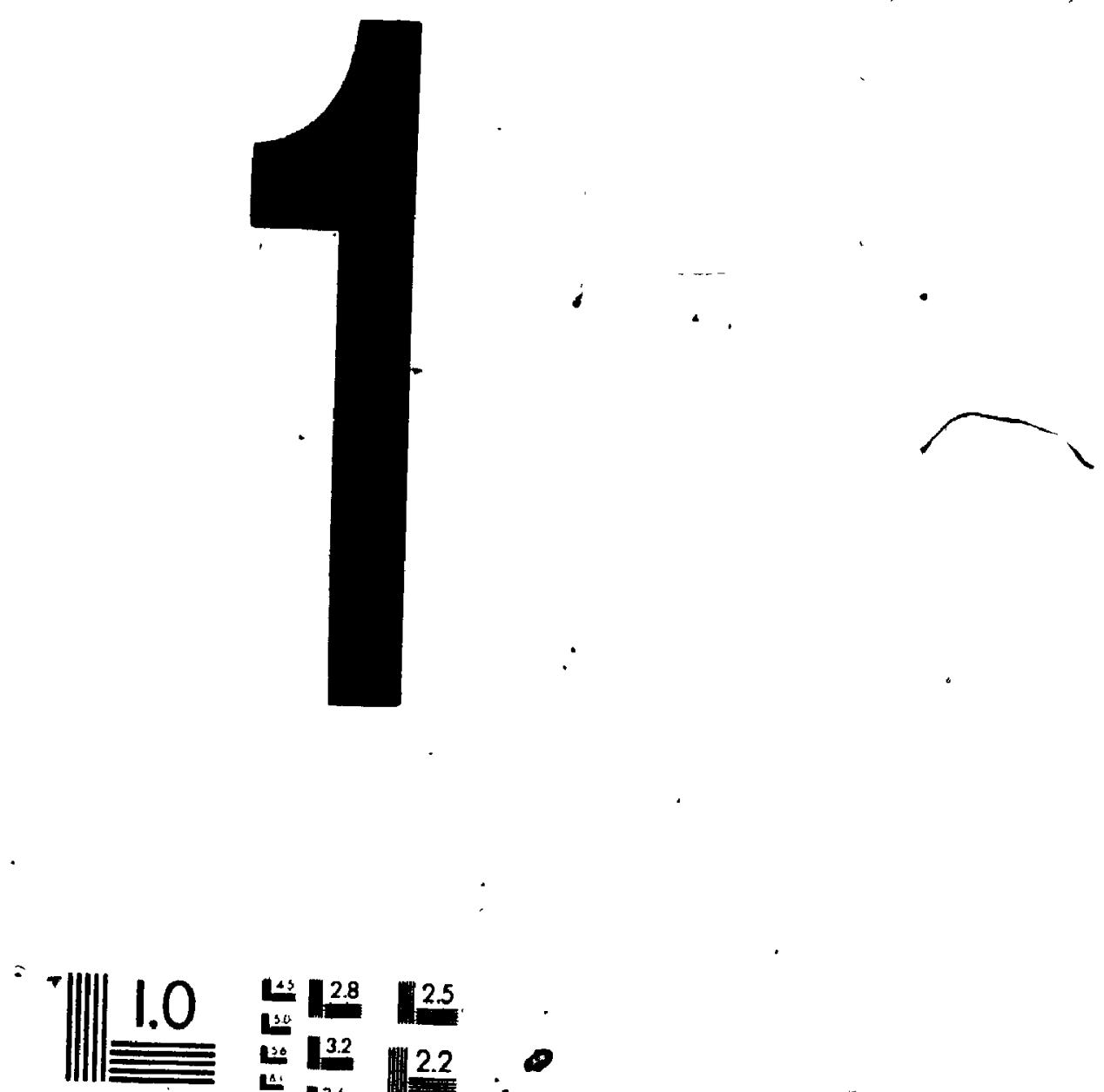

$$
\begin{aligned}
& \text { L4: } 2.8 \text { : } 2.5
\end{aligned}
$$

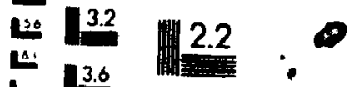

$$
\begin{aligned}
& \text { 는 } 14.0
\end{aligned}
$$

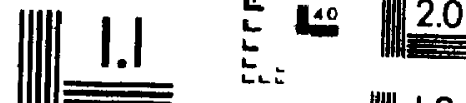

$\||| \mid 1.25$

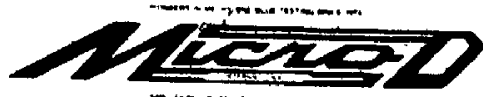

$\Delta$ 


\section{National Library}

\section{Canadian Theses Sęrice}

Ottawa, Canada

KIA ON4
Bibliotheque nationale du Canada

,

Service des thèses canadiennes. 
The Biosynthesis of Butenolide by Fusarium culmorum HLX 1503,

by

Jennifer E. Kendall, B.Sç.

(Carleton University)

. 
Permission has been granted to the National Library of Canada to microfilm this thesis and to lend or sell copies of the film.

The author (copyright owner) has reserved other publioation rights, and neither the thesis nor extensive extracts from it Nay be printed or otherwise reproduced without his/her written permission.
L'autorleation a sté accord $\dot{6}^{\circ}$ a la Bibliotheque nationale du Canada de icrofilmer cette these et de pretef ou de vendre des exemplaired du f11m.

L'auteur (t1túlaire du drolt d'auteur) se reserve 1 es autres droits de publication? ni la these ni de longs extraits de celle-cl ne doivent etre imprimbs ou autrement reprodults sans on autorleation ecrite.

$$
\text { I SBN } \quad 0-315-46298-1
$$




\section{Statement of Approval}

The undersigned recommend to the Faculty of Graduate studiés and Research to accept this thesis entitled:

The Blosynthesis of Butenolidè by Fusarium culmorum HLX 1503 submitted by Jennifer $E$. Kendall in partial fulfillment of the requirements for the degree of Master of Science.

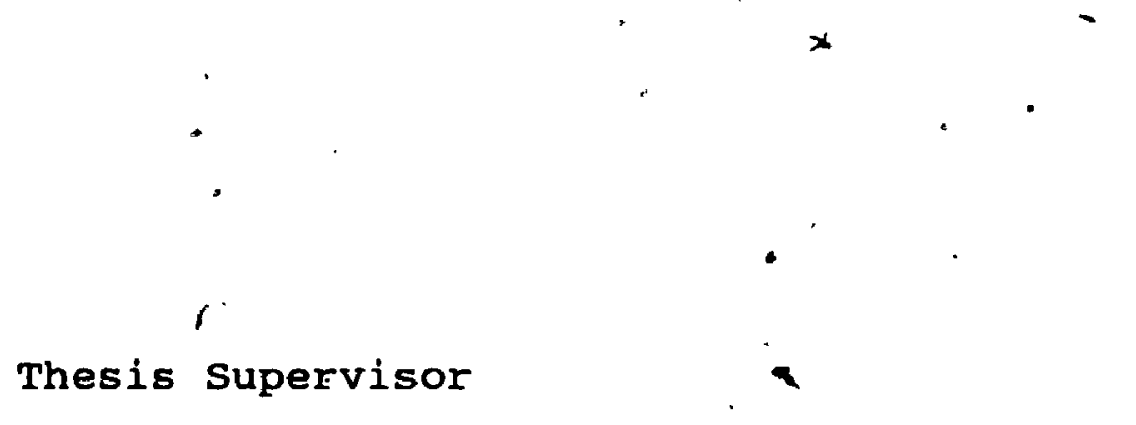

Thesis Co-supervisor 


\section{Acknowledgements}

I would like to thank Dr. J. W. Apsimon and Dr. R., Greenhalgh for their support and supervision throughout this project. I also appreciate the help and guidance of Dr. J. D. Miller in operating the fermenjor and helping me to understand the metabolism of fungi.

I would especially like to thank Dr. Barb"Blackwell for the time, guidance, support and encouragement she gave me with the project, particularly with the NMR work.

The nembers of the laboratories at Carleton (Lee Collier; Geoff Gay, David Fielder, Lisa Morrison, Doris Schaufhauser and Dr. Ted Edwards) and Agriculture Canada (Gary Montgomery, Mel Ewen, Pierre Lafontaine, Anne Ashley and Dr. Marc Savard) as wefl as stephane Dupont are much apprediated. They, and their antics, were good for a laugh when $I$ most needed $i t$.

I would also like to thank Gary Montgomety for his help with the GC/ion trap, Mel Ewen for explaining his HPLC systex to me and Pierre Lafontaine for the GC/MS and isotope ratio monitoring analysis work.

Finally, I would like to thank my family for their support and encouragement throughout this project. 


\section{Abstract}

The formation of butenolide by E, culmorum HLX 1503 in shake flask cultures and a $10 \mathrm{~L}$ stirred jar fermetor with respect to oxygenation and trichothecene formation is described.

The addition of $13 \mathrm{C}-1$ acetate, $13 \mathrm{C}-2$ acetate, ${ }^{13} \mathrm{C}$ glutamic acid and $15 \mathrm{~N}$ glutamic acid to the medium demonstrated that the biosynthetic origin of butenolide lies in the tricarboxylic acid (TCA) cycle and glutamic acid.

The activity of the TCA cycle and the mevalonic acid pathway was monitored through the incorporation of ${ }^{13} \mathrm{C}-2$ acetate into butenolide and 3-acetyldeoxynivalenol (3-ADON) and. the resultant enrichment patterns, as discerned by ${ }^{13} \mathrm{C}$ NMR spectroscopy; in each molecule. From the enrichment patterns it was determined that butenolide production, was initially faster than 3-ADON production and that once 3-ADON synthesis increased, butenolide synthesis decreased and eventually stopped. The finitiation of the synthesis of 3-ADON has been correlated with the depletion of the nitrogen source $(20)$. 


\section{Table of contents}

Statement of Approval

Acknowledgements

Abstract

Table of Contents

1-0 INTRODUCTION . . . . . . . . . . . . . . . . . . . . . . . 1 .

1-1 Secondary Metabolism, secondary. Metabolites and Mycotoxins.

1-2 Trichothecenes: The Fungi that Produce them and The Disorders Associated with them. . . . . . . . . . . 5

- A Comparison of Two Toxigenic Species of Eusaris and Their Toxic Metabolites . . . . . . . . . . . . . . . 8

$1-3$ Butenolide .. . . . . . . . . . . . . . . . . . . . 14

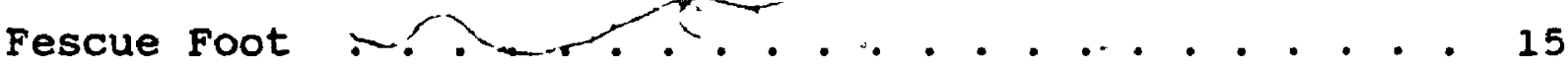

1-4 Biosynthesis of Butenolide . . . . . . . . . . . . . . 16

The Tricarboxylic Acid Cycle. . . . . . . . . . . . . 16

a) $13 \mathrm{c}$-Enriched Acetate .. . . . . . . . . . . . 18

b) ${ }^{13}$ C-Glutamic Acid . . . . . . . . . . . . . . . 26

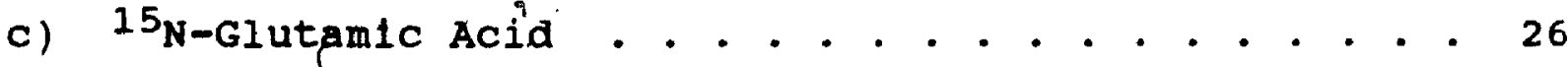

1-5 Degradation of Butenolidè ................. . . . 27

1-6 Purpose-ot Project . . . . . . . . . . . . . . . 27 


\section{2-1 GENERAL INFORMÁTION}

NMR Spectroscopy of crude Fungal Extracts and some Possible Methods of Analyses

Determination of Enrichment in Butenolide

$13 \mathrm{C}$ NMR Datà of Butenolide.

2-2 OPTIMIZATION OF GROWTH CONDITIONS, FOR MAXIMUM BUTENOLIDE

PRODUCTION.

The Effect of Oxygenation on Butenolide Production . . 34

The Significance of Dry Weight and pH Measurements. 36 Investigation of the Observed Variability in $25 \mathrm{~mL}$ Cultures.

2-3 OPTIMIZATION OF LLABELLING CONDITIONS. . . . . . . . 40

Incorporation of ${ }^{13} \mathrm{C}$ Acetate into Butenolide . . . . . 41

Maximization of Incorporation of Stable Isotope Precursors

Into Butenolide . . . . . . . . . . . . . . 48

2-4 IS GLUTAMIC ACID A METABOLIC PRECURSOR OF BUTENOLIDE? - 55

2-5 INVESTIGATION OF THE DEGRADATION OF BUTENOLIDE • • • • 66 Decline of Butenolide concentration in Shake Flask

Cultures

2-6 FERRENTOR EXPERIMENTS

Metabolism study - monitoring through the use of ${ }^{13} \mathrm{c}-2$

acetate.

Experiments to study the ${ }^{13} \mathrm{C}-2$ Acetate Incorporation into

the Metabolic Products of E, culmorum Hix 1503:

Experiment 1 (FR-1) 


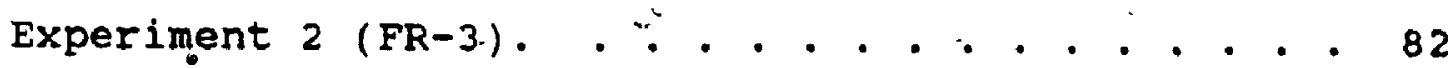

Fermentation Studies of Butenolide Degradation. . . . . 87

The First Experiment (FR-2) . . . . . . . . . . . 87

The second Experiment (FR-4) . . . . . . . . . . 90

${ }^{13} \mathrm{C}$ NMR of Whole cells and an Aqueous Extract of the Whole Cells from FR-4 . . . . . . . . . . . 93

1

3-0 EXPERIMENTAL TECHNIQUES - . . . . . . . . . . . . . . . . . 97

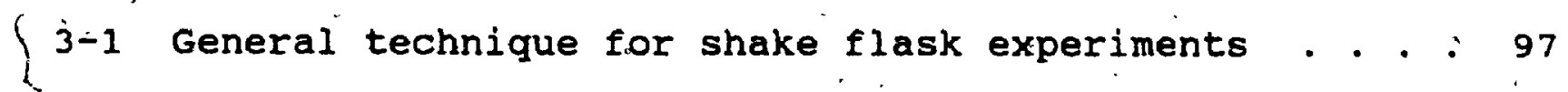

3-1a Tęchniqués for HPLC Quantitation of Butenolide

and 3-ADON, NMR Spectroscopy and Purification of

${ }^{13} \mathrm{C}$ Enriched Butenolide from Crude Fungal

Extracts. . . • . . . . . . . . . . . . . . . 98

3-2 General procedure for stirred jair fermentor

experiments. . . . . . . . . . . . . . . . . . . 100

3-3 Procedure for the precipitation of protein from mycelium.103

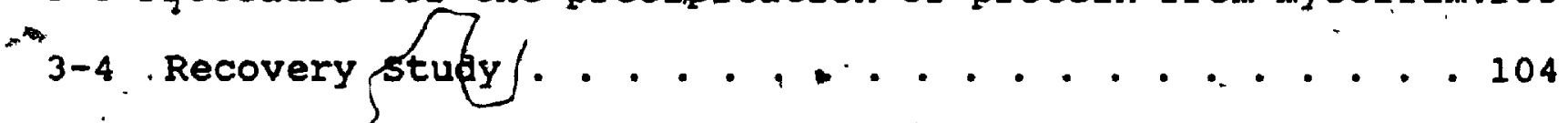

Conclusioñ . . . . . . . . . . . . . . . . . . . . . . . . 107

Suggestions for Future Work . . . . . . . . . . . . . . . . . . 110

- References : . . . . . . . . . . . . . . . . . . . . . 112

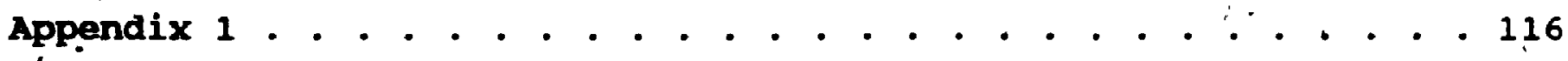


Calculations Used in the Analysis of ${ }^{13} \mathrm{C}$ NMR spectral Data Obtained from 10 L Stirred Jar Fermentor Experiments . . 116 The ${ }^{13} \mathrm{C}$ Resonances and Their Use in This Study . . . . . . 118 Calculation of Total ${ }^{13} \mathrm{C}$ Enrichment, at a specific Carbon Atoml19 Calculation of the Ratio of Single to Multiple Enrichment in Butenolide . . . . . . . . . . . . . . . . 121 Per Cent of Triple Enrichment in Butenolide as Determined by C-3 splitting Pattern Intensities . . . . . . . . . 123 Extent of Enrichment in 3-ADON ..............-123

Appendix 2 . . . . . . . . . . . . . . . . . . . . . . . . . 126 Metabolite Quantitation by High Performance Liquid Chromatography (HPLC) $\therefore .126$ Appendix 3 . . . . . . . . . . . . . . . . . . . . 129 ${ }^{13} \mathrm{C} /{ }^{12} \mathrm{C}$ Isotope Ratio Monitoring of ${ }^{13} \mathrm{C}$ Enriched Butenolide 129 


\section{List of Tables}

Table $i$. The effect of oxygenation on the level of butenolide observed in cultures of $E_{\text {e }}$ culmerum as indicated by $25 \mathrm{~mL}$ and $50 \mathrm{~mL}$ culture volumes.

Table 2. The concentration of butenolide found in $25 \mathrm{~mL}$ cultures of $\mathrm{E}$, culmorum $\mathrm{HLX} 1503$ with respect to dry weight, $\mathrm{pH}$ and time.

Table 3. Butenolide concentrations and $\mathrm{pH}$ values in shake flask cultures of $E$, culmorum HLX 1503 fed enriched and unenriched acetate and harvested at $48 \mathrm{~h}$ and $72 \mathrm{~h}$.

Table 4. The butenolide and 3-ADON concentratiohs of $F$, culmorum HLX 1503 cultures fed acetate and harvested at different times throughout the fermentation.

Table 5. The effect of adding glutamic acid to cultures of E. eulmerum HLX 1503 on the butenolide and 3-ADON concentrations.

Tabie 6. The effect of glutamic acid concentration on the production of butenolide and 3-ADON in

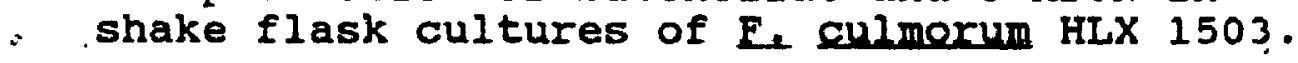

Table 7. Total ${ }^{13} \mathrm{C}$ enrichment factor in the carbon atoms of butenolide from the first fermentation experimert (FR-1).

Table 8. Ratio of multiple to single enrichment in butenolide enriched with ${ }^{13} \mathrm{C}-2$ acetate. Dată is from FR-1.

Table 9.. ${ }^{13} \mathrm{C}$ enrichment of ther adjacent carbon atoms of butenolide as compared with the enrichment in 3-ADON. Data obtained from FR-3.

Table 10. Recovery of butenolide from spiked media samples. 
List of Figures

fig. 1 Representation of the relationship between some of the major primary metabolic pathways that utilize acetyl coenzyme $A$ and the secondary metabolites produced through them.

fig. 2 From Miller and Blackwell (20). Mycotoxin production and nutrient supply in a $10 \mathrm{~L}$ stirred jar fermentation of $\mathrm{E}$. culmorum HLX 1503.

fig. 3 Trichodiene and the trichothecane nucleus.

fig. 4 structures of some sécondary metabolites produced by species of Fusaria.

fig. 5 The structure and-numbering system of

4-acetamido-4-hydroxy-2-butenoic acid

T-lactone (butenolide (1)).

fig. 6 Representation of the points of entry of

açetate into the TCA cycle and the resultant ${ }^{13} \mathrm{C}$ enrichment of the products of this cycle when ${ }^{13} \mathrm{C}-2$ acetate is incorporated and the glyoxylate shunt is not active:

fig. 7 The change in the enrichment pattern of

glutamic acid produced from the TCA cycle

when the glyoxylate shunt is active.

fig. 8 The observed enrichment pattern in

butenolide from incorporation of

${ }^{13} \mathrm{C}-1$ acetate $(\Delta)$ and ${ }^{13} \mathrm{C}-2$ acetate ( $)$ ).

fig. 9 The structure. and numbering system of

$3-A D O N(3)$. The carbon atoms arising from

${ }^{13} \mathrm{C}-1$ acetate $(\Delta)$ and ${ }^{13} \mathrm{C}-2$ acetate ( 0 ) are indicated.

fig. 10 Natural abundance ${ }^{1-3} \mathrm{C}$ NR spectrum of pure butenolide.

fig. $11{ }^{13} \mathrm{C}$ MMR spectra showing the incorporation of A) ${ }^{13} \mathrm{C}-1$ acetate and $\left.\mathrm{B}\right){ }^{1 \frac{3}{3}} \mathrm{C}-2$ acetate into butenolide (1). 
fig. $12{ }^{13} \mathrm{C}$ NMR spectra of the crude fungal extract from $50 \mathrm{~mL}$ shake flask cultures of $\mathrm{F}_{\text {culmorum }}$ HLX 1503 to which ${ }^{13} \mathrm{C}-2$ acetate "was added at several times.

fig. $13{ }^{13} \mathrm{C}$ NMR spectra showing the effect of harvest time on the incorporation of $13 \mathrm{C}-2$ acetate into butenolide (1) and 3-ADON. (3).

fig. 14. Is glutamic acid a precursor for butenolide biosynthesis?

fig. $15{ }^{13} \mathrm{C}$ NMR spectra of the crude fungal extract of $50 \mathrm{~mL}$ shake flask cultures of $\mathrm{F}_{\text {. }}$ culmorum ( $H L X 1503$ ) to which ${ }^{13} \mathrm{C}-5$ glutamic acid was added.

fig. 16, $15 \mathrm{~N}$ NMR "spectrum of the crude fungal extract to which ${ }^{13} \mathrm{C}$ glutamic acid and ${ }^{15} \mathrm{~N}$ glutamic acid were added.

fig. 17 Proposed biosynthetic route to butenolide from glutamic acid showing the incorporation of ${ }^{13} \mathrm{C}-5$ glutamic acid ( $(\square)$ and $13 \mathrm{C}-2$ acetate ( ) and the resultant enrichment pattern in butenolide.

fig. 18 Profile of butenolide and 3-ADON concentration. in $50 \mathrm{~mL}$ shake flask cultures of $\mathrm{E}$. culmorum HLX 1503 as a function of culture age.

fig. 19 Peaks and coupling constants for the three adjacent carbon atoms, $\mathrm{C}-2, \mathrm{C}-3$ and $\mathrm{C}-4$, of butenolide used for the calculations for level of enrichment.

fig. 20 Expansion of the $42 \mathrm{~h}^{13} \mathrm{C}$ NMR spectrum (fig. 23) showing the resonances used to determine the level of anrichment in 3-ADON.

fig. 21 The yield of butenolide and 3-ADON as a ifunction of culture age from FR-1.

fig. $22{ }^{13} \mathrm{C}$ NMR spectra of the crude fungal extracts of the first fermentation experiment of

E. culmorum HLX 1503 (FR-1).

fig. $23^{13} \mathrm{C}$ MR spectra of crude fungal extracts of , samples from FR-3. 
fig. $24{ }^{13} \mathrm{C}$. NMR spectrum of the precipitated protein from the myceisa of $\mathrm{E}$, culmorum $\mathrm{HLX} 1503$ (FR-2).

fig. $25{ }^{13} \mathrm{C}$ NMR spectra of the crude fungal extracts from FR-4 at various times.

fig. $26{ }^{13} \mathrm{C} \mathrm{NMR}^{\prime}$ spectra of homogenized whole cells from FR-4.

fig. $27{ }^{13} \mathrm{C}$ NMR spectrum of the aqueous extract from the final mycelia $(170 \mathrm{~h})$ of FR-4.

fig. 28 Experimental procedures for the growth of F. culmorum (HLX 1503) and the analysis of of the fungal medium.

fig. Al The resonances of $\mathrm{C}-2, \mathrm{C}-3$ and $\mathrm{C}-4$ of butenolide with the coupling constants indicating which satellites are associated with each carbon atom.

fig. A2 Expansion of the region of the ${ }^{13} \mathrm{C}$ NMR

spectrum and the resonances used to determine the level of enrichment in 3-ADON. 


\section{1-0 INTRODUCTION}

The pathogenic nature of certain species of fungi, to plants has been observed virtually since the beginining of agriculture. These plant pathogens often produce metabolites which adversel'y affect the human and animal populations when they are ingested. There are several examples in recent history where this property has been observed. In 1960, Turkey ' $X$ ' disease killed 100,000 turkeys, 14,000 ducklings and thousands of partridge and pheasant poults in England. There was an outbreak of hepatitis in western India in 1974 in the human. population. In the mid 1930's and late 1970's there were outbreaks of a sickness in horses called equine leucoencephalomalacia (ELEM) in the United States (1) and alimentary toxic aleukia has been responsible for. the distress and death of thousands of people since it was first recorded in the $19^{\text {th }}$ century (2). Although these syndromes are ali very different, they have one thing in common; they are all caused by mycotoxins.

Mycotoxins are metabolites that are produced by fungi growing on cereal crops, nuts, soybeans and several other crops including apples. The Turkey ' $\mathrm{X}$ ' disease outbreak in England was traced to contaminated nuts from Brazil and led to the discovery of". 
aflatoxins. The patitis outbreak in India and the Turkey ' $X$ ' disease are both thought to have been caused by aflatoxins, which are produced primarily be Aspergillus flavus Link and A. parasiticus speare. The equine leucoencephalomalacia is probably caused by toxins from Eusarium monififorme and alimeritary toxic aleukia is thought to be caused by the trichothecene mycotoxins; since mycotoxin producing fungi grow on some of the staple foods of both humans and animals, both populations are affected by them. Also, products such as eggs, milk, dairy products and meat can be contaminated through the ingestion of feed containing mycotoxins. Therefore, the problem of, mycotoxins is pertinent to the human as well as the animmal, populations.

1-1: Secondary Metabolism, Secondary Metabolites and Mycotoxins

Secondary metabolism is best defined in terms of its normal growth of the cells of an organism such as sugars, amino acids, nucleotides, fatty acids and polymers derived from these molecules. In all living organisals, primary metabolism provides energy, 
synthetic intermediates and key macromolecules for the organism. Organisms exist in, primary metabolism until a nutrient limitation is experienced at which point secondary metabolites may be produced: These metabolites encompassing an enormous variety of chemical structures are produced from intermediates of primary metabolic pathways. Figure 1 is a representation of some of the relationships between primary and secondary metabolism. It is not evident whether or not secondary metabolism is essential for the life of the organism or the cell engaged in it: secondary metabolism exists in many organisms but the term has a different meaning for different organisms. In fact, secondary pathways are unique to a species or family of closely related organisms $(3,4)$ and are sometimes even strain specific in fungi. The products of these pathways do not benefit the cell that produces them but rather the organism as a whole (6) and are a -product of cell specialization. Some examples of secondary metabolites include perfumes such as geraniol or rose oil, caffeine, insect pheromones, poisons such as strychnine or mycotoxins, antibiotics and medicines (ie. ephedrine for use in respiratory ailments) natural narcotics and hallucinogens, and many more (5).

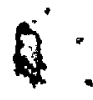




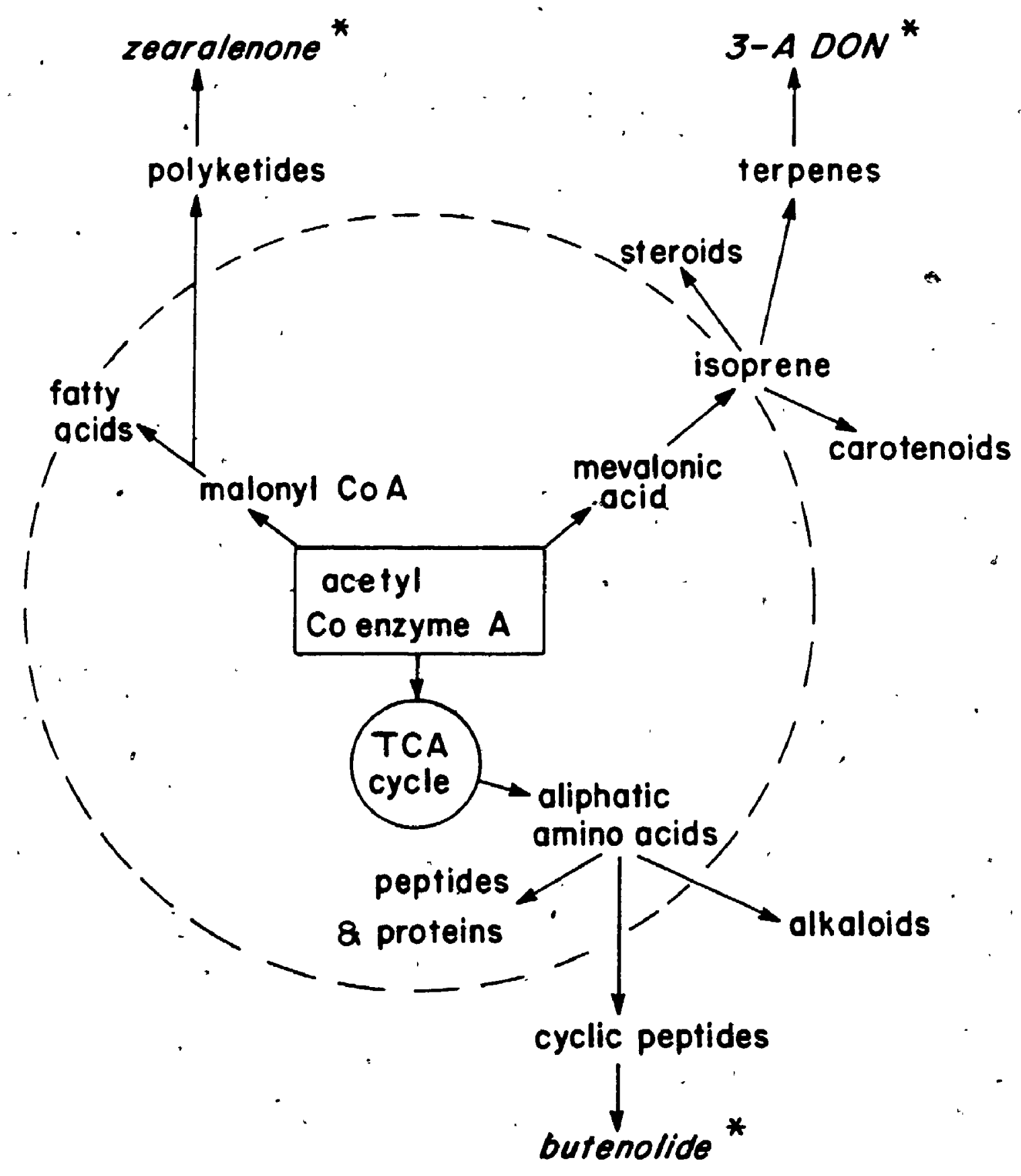

fig. I Representation of the relationship between some of the major primary metabolic pathways that utilize acetyl. coenzyme $A$ and the secondary metabolites produced through them. The dashed line indicates a boundary between primary (inside) and secondary (outside) metabolism and the * symbol denotes example mycotoxins. 
Mycotoxin is a term used to describe secondary metabolites that are produced by filamentous furigi and have detrimental biological effects on animals and/or humans (3). This definition, however, does not cover the full scope of mycotoxins since some mycotoxins, such as some peptide toxins, are not necessarily secondary metabolites. Mycotoxins, as a.class of compounds have no molecular features in common. They are also not a chemical category of their own. The variety that exists in these molecules makes them very difficult to classify. The method of classifying them most widely used defines them in terms of their biosynthetic origin (7). Mycotoxins can, therefore be categorized in terms of the primary metabolic pathways from which the mycotoxin precursor was formed, ie the shikimic acid pathway, the tricarboxylic àcid cycle (TCA cycle) or the mevalonate pathway, which pads to terpene synthesis.

1-2 Trichothecenes: The Fungi that Produce them and The Disorders Associated with them.

The trichothecenes are a family of secondary. metabolites produced by several genera of Deuteromycetes including Eusarium, Trichothecium, Myrothecium and stachybetrys. These fungi are 
pathogenic to plants including those that produce cereal grains and are supplied as feedstuffs to farm animals. Eusarium, for example, was first discovered and classified by Link in 1809 ( 8 ). Since, then there has been much difficulty in the classification of Eusarium species with many of them being reclassified throughout the $20^{\text {th }}$.century. "There are 203 toxigenic strains of Eusarium species in 10 sections (9). These plant pathogens result in a reduction of crop yield and grain quality. Grain destined for human consumption must sometimes be downgraded to animal feed or discarded completely. Some of the 1980 Ontario white winter wheat was downgraded due to high toxin levels from Eusarium graminearum. Sproüting of the kernels and a large amount of pink discolouration had been notidea on the harvested grain. After analysis of samples from grain elevators and boats loading for export, it was discovered that there wh contamination of much of the wheat by deoxynivalenol (vomitoxin) (11). Some samples were found to contain up to $8.5 \mathrm{ppm}$ of vomitoxin (11). The contaminated wheat was either destroyed or sold as animal feed depending on the extent of the contamination.

The trichothecene mycotoxins produced by the toxigenic Eusaria are the cause of many illnesses in humans and animals throughout the world. The red-mold 
or Akakabi toxicoses and bean-hull poisonings in Japan, the grain toxicoses in the United States, China, Japan, Korea, the U.S.S.R. and Canada, and the stachybotryotoxicoses in Central Europe $(10,12,13)$ are some exämples of disorders attributed to the trichothecenes. Inception of Al-ipentary Toxic Aleukia (ATA, T-2 poisoning), or septic angina is characterized by a burning sensation in the mouth; esophagus and stomach after ingestion of contaminated, food. The symptoms develop into emesis," nausea, fever, weakness, skin inflammation, and necrosis, destruction of bone marrow, haemorrhage and death (14). If the disease is caught early enough the patient may survive provided the contaminated food can be removed from their diet. Even though ATA has been recorded since the $19^{\text {th }}$ century, there was an outbreak the U.S.S.R. in 1932 and, in the mid 1940's, Russia saw many of its people suffer from this disease (2). It is an illness that was prevalgnt during world war II particularly among the peasants, who, because of the shortage of food caused by the war, wefe forced to eat overwintered crops and theit products such as bread: The overwintered crops are a good medium for the -. propagation of the Eusarium fungi.

Eusárium toxicoses are manifested slightly differently in animal populations than in humans. One 
of the trichothecenes, deoxynivalenol (DON), is reported as being a possible cause of the feed refusal 'responses in swine (8). T-2 toxin, a highly toxic trichothecene, also elicits the feed refusal response in swine and cattle. The clinical effects of these toxins has been grouped into four categories: 1) feed refusal, 2) dermal necrosis, 3) gastroenteric effects and 4) coagulopathy and are generally similar among most species tested ( 1 ). Another toxicose, known as fescue foot, causes the hooves and tails of cattle to become gangrenous and fall off. This disease has not been conclusively connected to trichothecenes and it is suspected that one of the causative agents is butenolide (1), a non-trichothecene toxin produced by some species of Eusaria.

A Comparison of Two Toxigenic Species of Fusaria and Their Toxic Metabolites

Each species of Eusarium produces a distinct variety of mycotoxins. Although the same toxins may be produced by different species the proportions in which they are produced will be peculiar to a species. E, culmorum and $E_{2}$ graminearum are both members of the section Discolor and both produce butenolide (1), 3-acetyldeoxynivalenol (3-ADON) (3), deoxynivalenol 
(DON), diacetoxysoirpenol (DAS), neosolaniol and zearalenone (a metabolite similar to estrogen). E. culmorum strains, however, produce calonectrin and culmorin. type molecules whereas $E$ graminearum produces fusarenon-X (9) (see fig. 4 for structures of the above mycotoxins). Both of these species also produce other secondary metabolites. $F_{\text {e }}$ culmorum is one of the pathogens responsible for the moldy grain toxicoses mentionepd earlier and $B$ graminearum produced the akakabi or red mold toxicoses of Japan.

Under varied experimental growth conditions, the production of selected classes of toxins can be somewhat controlled. The production of these secondary metabolites is triggered. by a stress on the fungus, usually a depletion or limitation of a nutrient source and a drop in $\mathrm{pH}$. The relationship between ammonium, sucrose and glucose concentration and the production of

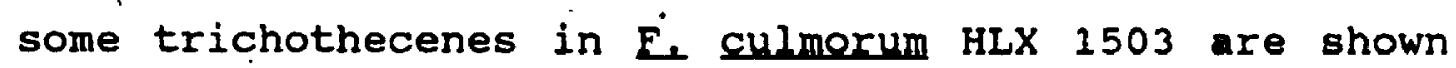
in figure $2(20)$. As, the nitrogen and glucose sources are depleted, the production of the secondary metabolites is initiated. The sucrose is broken down into glucose and fructose and the glucose is then used as an energy source. Increased trichothecene production can be directly related, to the inavailability of nitrogen (fig $2,36 \mathrm{~h}$ ). 


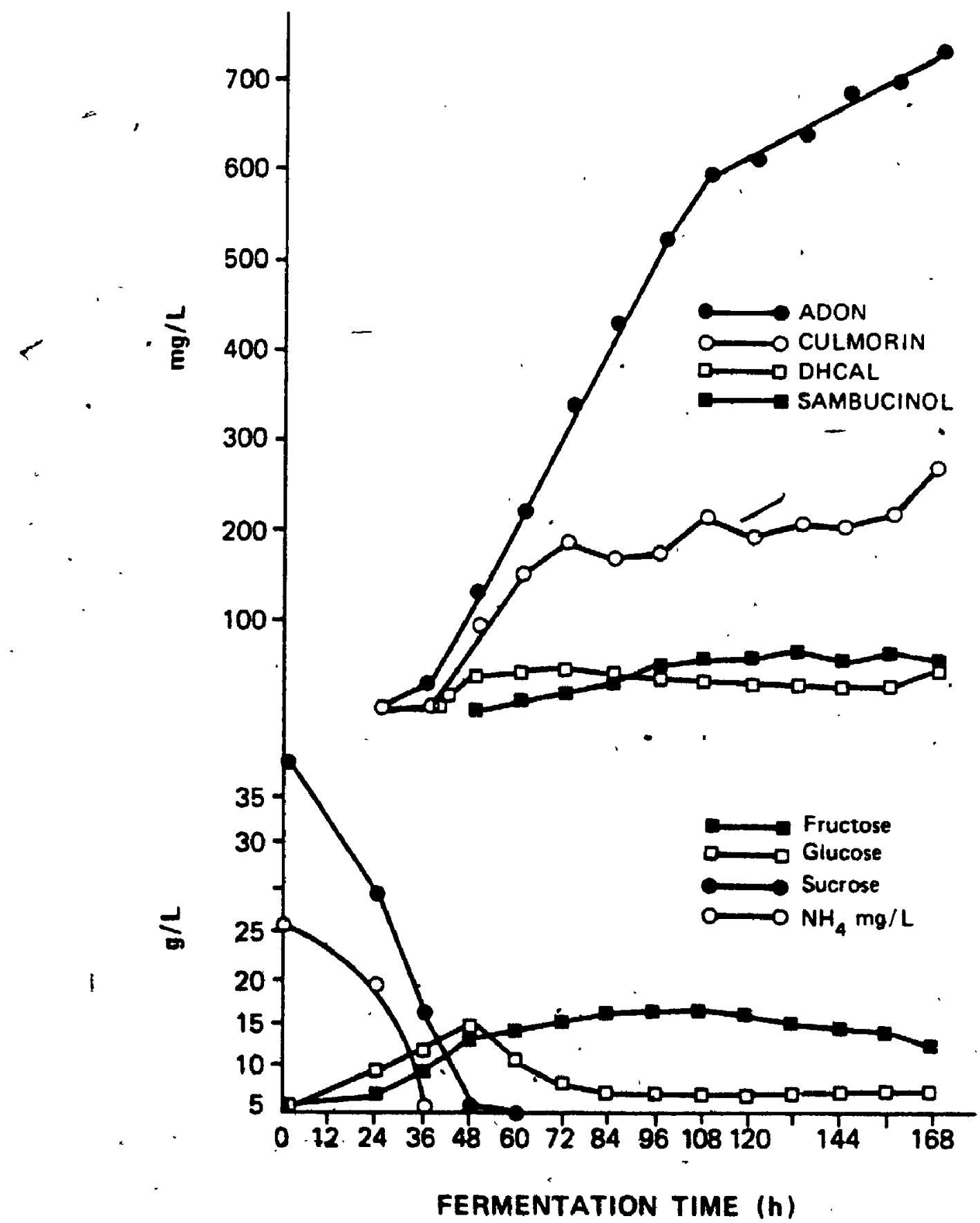

fig. 2 From Miller and Blackwell (20). Mycotoxin production and nutrient supply in a $10 \mathrm{I}$ stirred far fermentation of $F$. culmorum HLX 2503. 
Structural Characteristics of the Trichothecenes

The trichothecenes are sesquiterpenoid in origin as suggested by the number of carbons in the tetracycltc skeleton. They are alcohol or ester derivatives of the trichothecane nucleus (fig. 3 ).

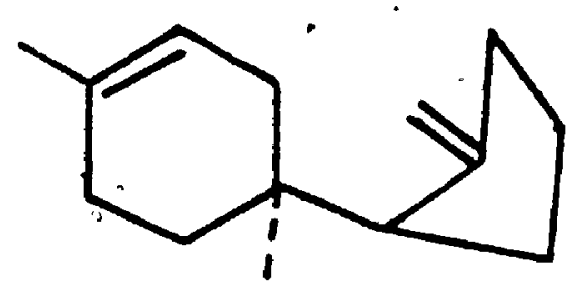

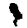

Trichodiene

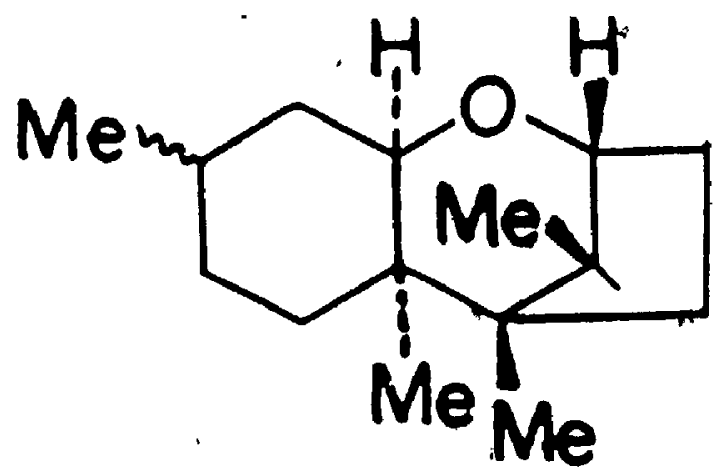

trichothecane

fig. 3 Trichodiene and the trichothecane nucleus.

There are over 80 known naturally occurring trichothecenes of three different basic ring structures. Th $\mathrm{A}^{\mathrm{T}-2}$ toxin type trichothecenes possess an additional substituent group at $c-8$ which, in $T-2$ 
toxin is leucine derived side chain. The third group of trichothecenes have a macrocyclic ring from $\mathrm{C}-4$ to C-7. The variation within the trichothecene groups occurs primarily at positions $\mathrm{c}-3, \mathrm{C}-4, \mathrm{C}-7, \mathrm{C}-8$ and 'c-15 where alcohol or ester groups may be added or removed. Almost all of the known structures of the naturally occurring compounds of this family possess an olefinic bond between $c-9$ and $c-10$ and an epoxy group at. $c-12$ and $c-13 ;$ hence, the name 12,13-epoxytrichothec-9-ene or more'simply. trichothecene. These molecules have their biosynthetic origin in the mevalonic acid pathway from terpene synthesis. Trichodiene (fig. 3), a common intermediate of the trichothecenes, has been shown to be formed from mevalonate through the enzymatic cyclization of trans-trans-farnesyl pyrophosphate (15). Several examples of trichothecenes and other secondary metabolites produced by Eusarium, species are illustrated in figure 4. 


\section{B. CULMORIN}

A. TRICHOTHECENE SKELETON

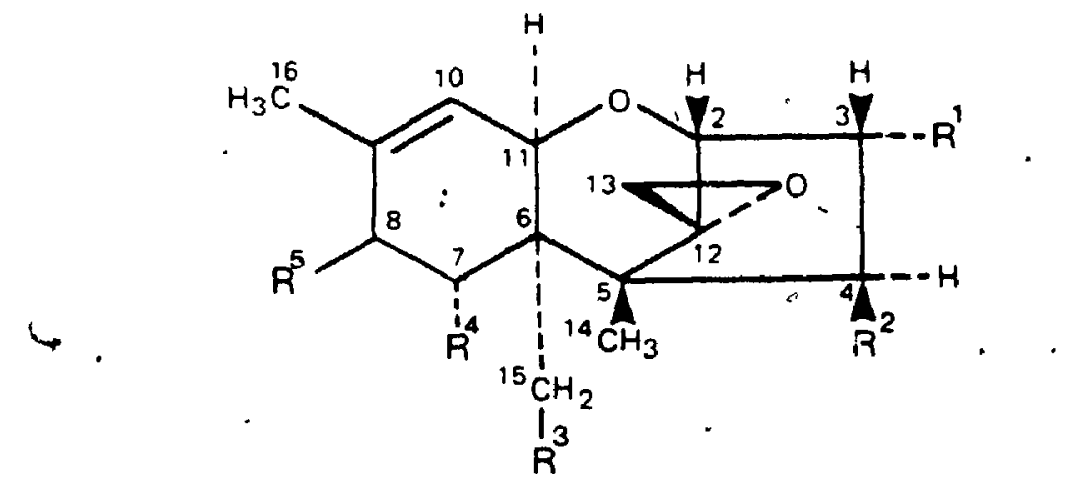

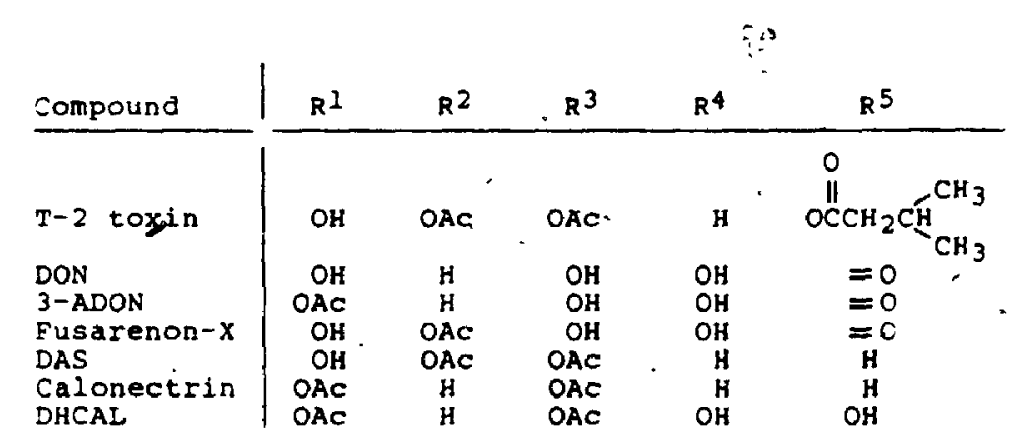

\section{SAMBUCINOL}

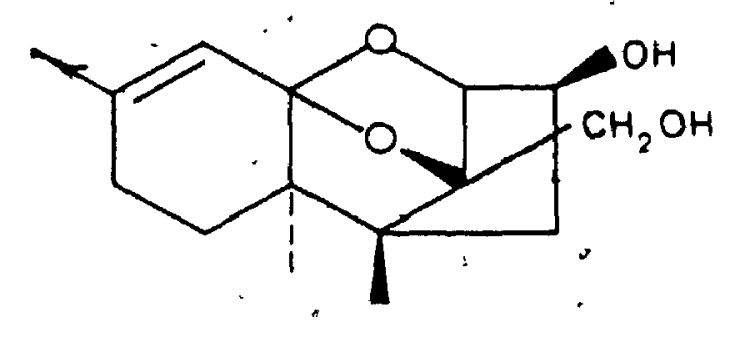

D. ZEARALENONE

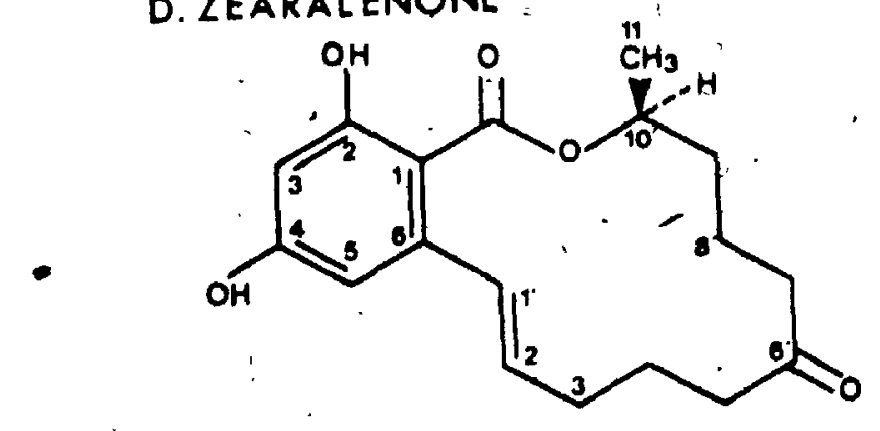

fig. 4 Structures of some secondary metabolites produced by species of Fusaria. The numbering system is typical of the trichothecene mycotoxins. 


\section{1-3 Butenolide}

Butenolide (1) is the common name for another secondary metabolite produced by several species of Fusarium. It is produced earlier in the fermentation of Eusarium than the trichothecenes but seems to be produced in conjunction with them. The structure of 4-acetamido-4-hydroxy-2-butenoic acid T-lactone, commonly referred to as butenolide (1), is shown in fig. 5 .

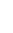

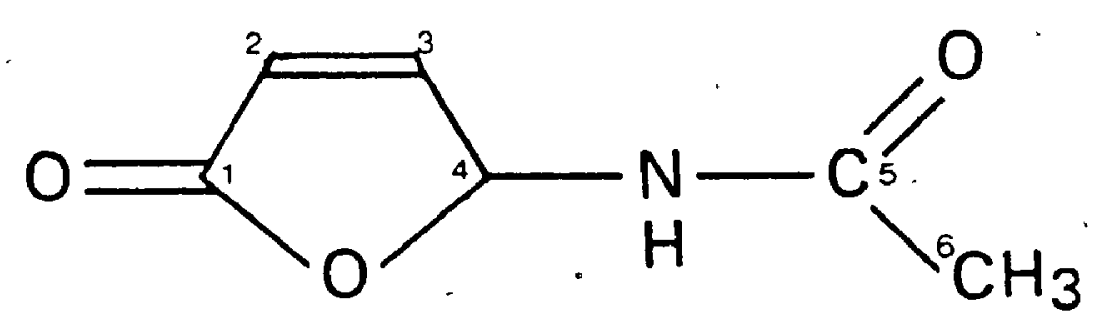

(1)

fig. 5 The structure and numbering system of 4-acetamido-4-hydroxy-2-butenoic acid 1-lactone (butenolide (1)).

This molecule was discovered independently in three different laboratories in 1967 , and 1968 while 
researchers were investigating the cause of fescue foot (16). They were looking for trichothecenes when butenolide was discovered. Butenolide (1) is easily detected by three strong absorptions in the infrared region at $1705,1760,1780 \mathrm{~cm}^{-1}$ and at $202 \mathrm{~nm}$ ih the ultraviolet region $(13,16)$. Although there is a chiral carbon at the $T$-carbon (position 4) there is no optical rotation observed $(13,16)$.

Fescue Foot

Fescue foot is a non-oontagious disease found in cattle grazing on pastures of tall fescue grass. In the United-States the disease is-Iimited to the bovine population but in Australia and New Zealand it is also evident in sheep (17). It is still, a major problem in . Missouri in certain years with symptoms appearing as early as one week after exposure depending on the toxicity of the grass. Fescue foot is characterized by loss of weight, arched back, rough coat, lameness in the hind quarters, dry gangrene of thie extremities (usually hooves and tails), and sloughing of these extremities $(13,18)$. Outbreaks of the disease are correlated with high nitrogen, fertility and is "host common when the temperature is about $5^{\circ} \mathrm{C}$. The fungus that causes fescue foot proliferates in the yearly 
accumulated growth of the tall fescue grass. The only treatment for the illness is to remove the animal from the infected pastures. This can severely limit the grazing range of the herds. In Missouri, field reports have indicated that between the years 1966 and 1973 , $10 \%$ to $30 \%$ of the herds were affected with fescue foot and within any one herd the rate of infection ranged from a single animal to the whole herd (17).

1-4 Biosynthesis of Butenolide

To date, the biosynthesis of butenolide (1) has not been studied in detail although in 1977 vesonder et al. (19) suggested that 4-acétamido-2-butenoic acid may be an intermediate. This butenoic acid was isolated from E. graminearum and was suggested as a precursor to butenolide based on their structural similarity. No further' studies' to this effect were published.

The Tricarboxylic, Acid Cycle

The fricarboxylic acid (TCA) cycle is a major. pathway for. the production of aliphatic amino acids in all living systems. It is most active during primary metabolism in fungi and slows down when these organisms enter secondary metabolism : During seconda'ry 
metabolism the fungus is not able to spare the energy required to use the TCA cycle producing amino acids. Therefore, the amino acids are produced when there are sufficient nutrients available to support growth i.e. primary metabolism. From the structure of butenolide (1), it is reasonable to suggest that it is produced from glutamic acid (2), an amino acid derived from $\therefore$ $\alpha$-ketoglutaric acid, a key intermediate of the I'CA cycle.

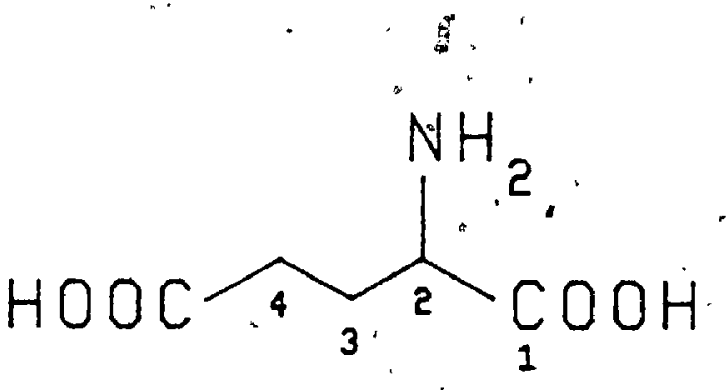

Glutamic acid

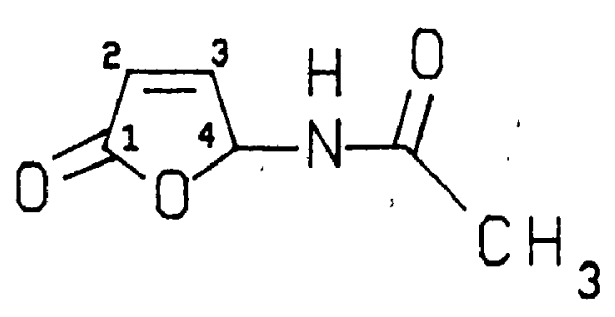

Buteniolide 
Also, butenolide (1) is produced as the fungus approaches nitrogen limitation $(20)$, which is] one of the factors that triggers the end of primary metabolism . and the initiation of secondary metabolism. By contrast, the trichothecenes are not produced until the nitrogen source, ammonium, declines to trace amounts $=(20)$. Therefore, since the production of butenolide is highest when the TCA cycle is operating normally there is a possibility that it is produced from a product of this pathway.

a) $13 \mathrm{C}-$ Enriched Acetate

The study of secondary metabolites through ${ }^{13} \mathrm{C}$-enriched precursors has become more efficient and feasible since 1970 when advances in nuclear magnetic resonance (NMR) spectrometers enabled móre rapid and sensitive acquisition of ${ }^{13} \mathrm{C}$ NMR spectra possible (21). The ${ }^{13}$ C-enriched biological precursors required for these types of studies were also more abundant by this time. The enriched precursor used in this study was $13 \mathrm{C}$-acetate becáuse, it is an ideal precursor for studying' both the mevalonic acid pathway and the TCA cycle products and it is easily obtained, relatively inexpensive and completely absorbed by the organism: 
Acetate is utilized in the TCA cycle at two points (fig. 6). Each of these are possible points of entry $y$ for enriched acetate molecules when they are added to the fungal growth, medium., The enrichment pattern of glutamic acid (2) from both ${ }^{13} \mathrm{C}-1$ acetate and $13 \mathrm{C}-2$ acetate as. it is produced from the TCA cycle is outlined in figure 6. As figure 6 indicates, there is an interesting and peculiar pattern that is produced when the same enriched molecule remains in the cycle for more than one turn.

The pattern of enrichment at positions 4,3 and 2 , successively, of glutamic acid varies when the symmetry at succinic acid is observed. The enriched methyl group of the acetate will then cause enrichment in either $c-3$ or $c-2$ after the first turn around the cycle rather than just $\mathrm{C}-3$ as indicated in figure 6 . This increases the possiblility of enrichment occuring in C2 of glutamic acid (2), which becomes $c-4$ of butenolide (1). 
fig. 6 Representation of the points of entry. of acetate into the TCA cycle and the resultant 13c enrichment of the products of this cycle when ${ }^{13} \mathrm{C}-2$ acetate is incorporated and the glyoxylate shunt is not active. The open symbols indicate that the label has survived at least one complete pass of the TCA cycle and has returned to the point where acetyl COA enters. The $\triangle$ indicates the first ${ }^{13} \mathrm{C}-2$ acetate to enter the cycle; the indicates the second acetate to enter the cycle and the

- indicates the third entry of an enriched acetate. Therefore, for the first pass around the cycle, follow the closed triangle. The closed triangle can either leave the cycle at $\alpha$-ketoglutaric acild or continue back to citric acid. Once back to citric acid, the triangle becomes open and the closed square becomes the next acetate molecule added. The same pattern can be followed for the square and the circle to yield the final enrichment patterns in glutamic acid. 


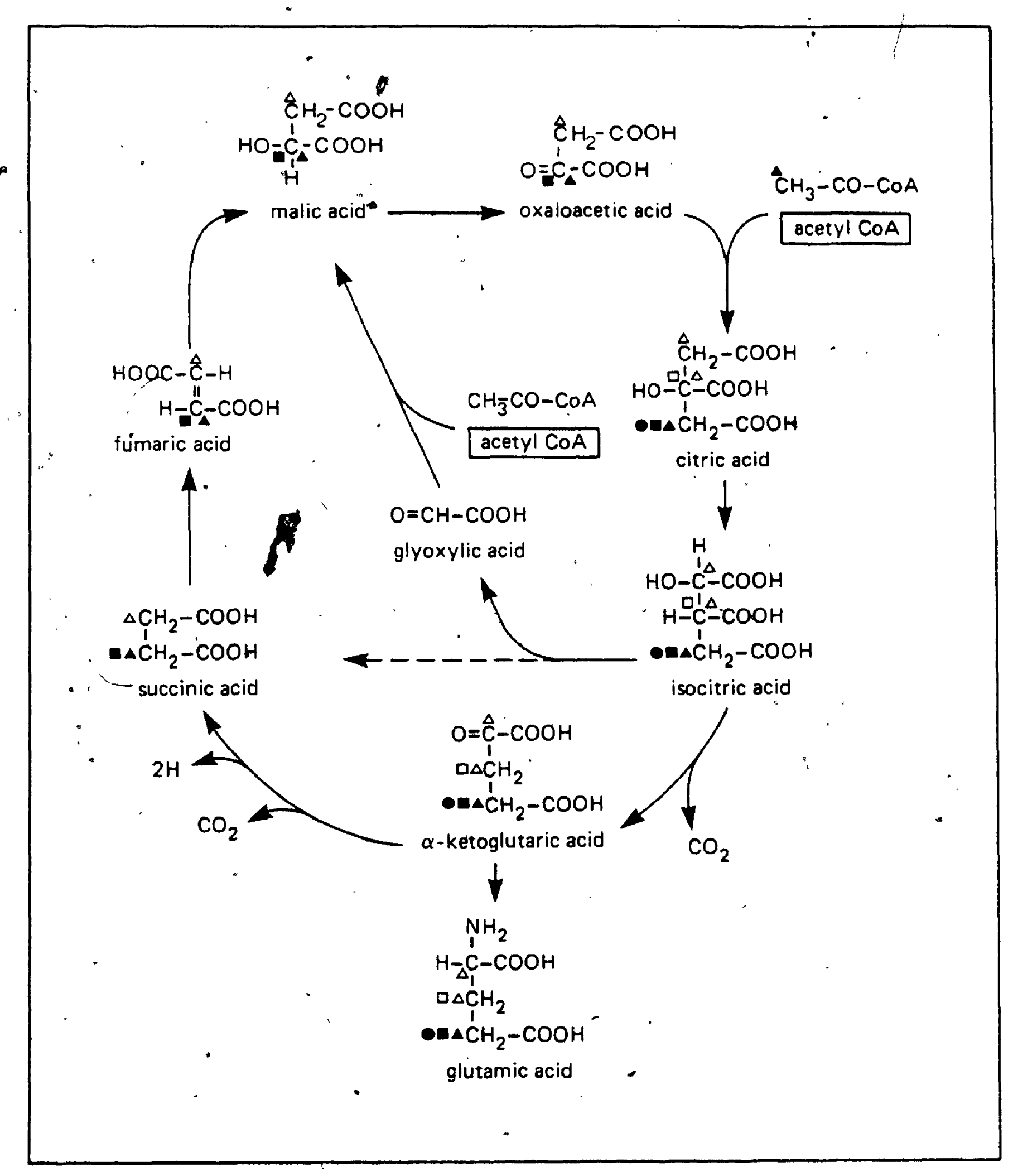


The incorporation of ${ }^{13} \mathrm{C}_{2}-1,2$ acetate into glutamic acid (2) has been reported to give the expected enrichment in the $\mathrm{C}-4$ and $c-5$ position of glutamic acid (2) as discerned from the incorporation into $\mathrm{C}-1$ and $c-5$ of thienamycin (22). The enrichment at $c-4$ and $c-5$ of 'glutamic acid (2) is characteristic of òne turn of the TCA cycle (fig. 6).

In fungal systems, there is a glyoxylate shunt that operates across the TCA cycle (23) effectively cutting the cycle into two pathways. when the glyoxylate pathway is operating, there is an additional point of entry of acetate. This changes the enrichment pattern of glutamio acid (2) as shown in figure 7. Again, the symmetry at succinic acid will cause the enrichment at carbon 3 and carbon 2 of glutamic acid to be of equal proportions for those molecules which enter that section of the glyoxylate shunt.

From the ratios and characteristics of multiple enrichment in the final products, the efficiency and activity of the TCA cycle and glyoxylate shunt can be determined. The determination of these features of the metabolism are made possible through calculations performed with ${ }^{13} \mathrm{C}$ NMR specting data of ${ }^{13} \mathrm{C}$ enriched crude fungal extracts when compared with a natural abundance standard spectrum. 


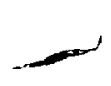

fig. 7 The change in the enrichment pattern of glutamic acid produced from the TCA cycle when the glyoxylate shunt is active. The enrichment pattern due to the incorporation. of ${ }^{13} \mathrm{C}-2$ acetate from the glyoxylate shunt is shown by a $a$ The $\Delta$ and the indicate enrichment from the TCA cycle entry point of acetate. The $\Delta$ is the first ${ }^{13} \mathrm{C}-2$ acetate incorporated and the 0 is the second from this point of entry. Again the open symbol denotes a molecule which has survived one full pass of the cycle. Note that the closed triangle moves directly from isocitric acid to succinic acid via the glyoxylate shunt and is not shown in $\alpha$-ketoglutaric acid. 


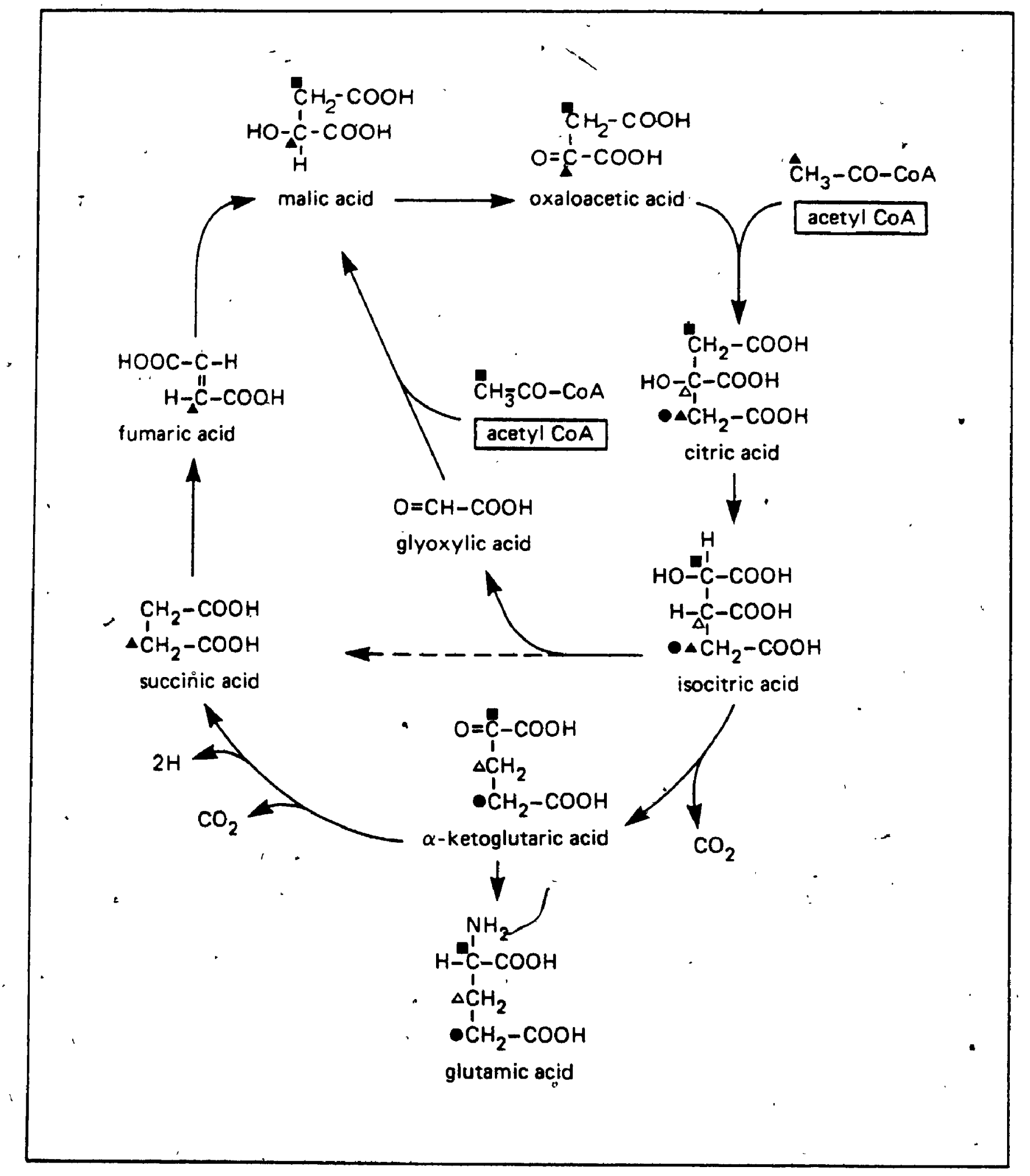


Through the use of ${ }^{13} \mathrm{C}$-acetate, labelled at either the 1- or the 2-position, we discovered (24) that buttenolide (1) had a labelling pattern consistent with the products of the TCA cycle. The carbon atoms of butenolide arising from $\mathrm{C}-1$ of acetate and $\mathrm{C}-2$ of acetate are shown in figure 8 . Carbons 2,3 and 4 are derived from $\mathrm{C}-2$ of acetate which is consistent with the condensation of acetyl-COA units with the loss of $\mathrm{CO}_{2}$ as found in the TCA cycle (fig. 6).

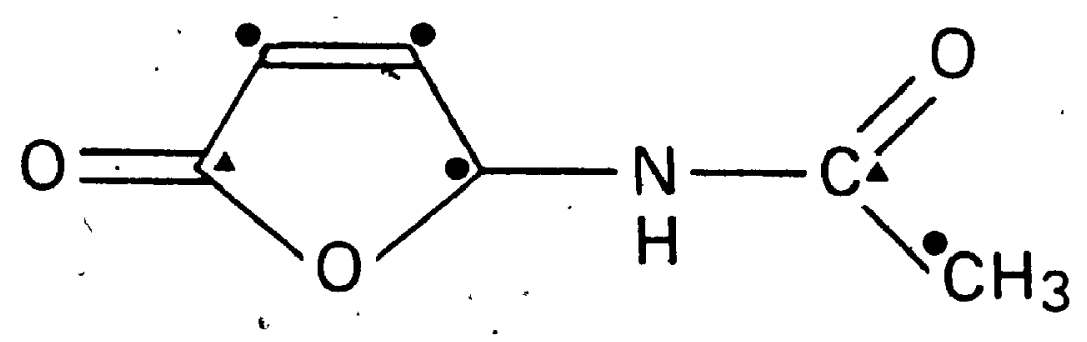

fig. 8 The observed enrichment pattern in butenolide from incorporation of $13 \mathrm{C}-1$ acetate $(\Delta)$ and $13 \mathrm{c}-2$ acetate $(\bullet)$. 
b) 13 C-Glutamic Acid

To determine whether or not glutamic acid (2) is a precursor of butentide (1), ${ }^{13} \mathrm{c}-5$ glutamic acid can be added to the fungal culture medium. The effect on the butenolide concentration and the subsequent incorporation into butenolide will give information as to whether or not this product of the TCA cycle is a precursor in the biosynthesis of butenolide.

c) $15 \mathrm{~N}-$ Glutamic Acid .

If glutamic

If glutamic acid is a precursor of butenolide the source of the nitrogen in butenolide ( 1 ) is likely to be glutamic acid (2). This can be deteryined by the use of ${ }^{15}{ }^{\prime}$ NMR through the incorporation of ${ }^{15} \mathrm{~N}$-glutamic acid. This would also show that glutamic acid (2) was a precursor. However, ${ }^{15} \mathrm{~N}$ NMR is difficult to perform since the natural abundance of ${ }^{15} \mathrm{~N}$ is only $0.36 \%(21)$. For 15 N NMR to be successful, it is much more effective to use ${ }^{15} \mathrm{~N}$-enriched precurșors. ${ }^{15} \mathrm{~N}$ NMR has successfully been used to confirm the structure of neosaxitoxin (25) and to follow the nitrogen assimilation and amina acid biosynthesis in intact mycelia of senococcum graniforme (26). 


\section{1-5. Degradation of Butenolide}

Earlier studies of the fermentation of $E$. curmerum HLX 1503, have shown that the concentration of butenolide is high at $4 \mathrm{~h}$ and declines to a, relatively low value by $78 \mathrm{~h}(20)$. This possible degradation of the molecule is interesting as it is "not widely reported in fermentation studies. Since butenolide is produced before the onset of secondary metabolism and it contains a nitrogen atom, the organism may use this metabolite as a nitrogen source later in the metabolism of the culture. This aspect of butenolide metabolism is of interest and can be studied along with the biosynthesis. Through NMR analysis of the crude fungal extracts of the fermentation medium, the biosynthesis and decline of butenolide can easily be studied. The relationship to trichothecene metabolism can also be investigated in this manner.

\section{1-6 Purpose of Project}

The aim of this project was to investigate and optimize the growth conditions of Eusarium culmerum HLX 1503 to obtain the maximum production of butenolide. Through this, the biosynthesis and further metabolism of this molecule would be determined. In optimising 
(

the growth conditions, the effect of oxygenation, growth time, $\mathrm{pH}$ and the mycotoxin profile of the organism would be studied. Once, this was accomplished, the metabolism of butenolide was to be studied by the use of precursors enriched with stable isotopes for the use of NMR spectroscopy. Since the metabolism of butenolide is so closely tied to the biosynthesis of the trichothecene mycotoxins, this relationship would also be studied. The incorporation of enriched precursors of both butenolide and the trichothecenes would be studied and interpreted in relation to the metabolic activity. of the fungus. 


\section{2-0 RESULTS AND DISCUSSION}

\section{2-1 GENERAL INFORMATION}

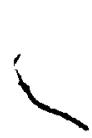

The study of butenolide (1) is of interest because this mycotoxin is postulated to be produced from a product of the TCA cycle, possibly in conjunction with the trichothecene mycotoxins. With the fermentation ' and analysis procedures used in this study we are able to monitor the dynamics of the production of butenolide . (1) and the trichothecene mycotoxins. The TCA cycle and the mevalonic acid pathway can be monitored simultaneously through the use of ${ }^{13} \mathrm{C}$ enriched acetate and ${ }^{13} \mathrm{C}$ nuclear magnetic resonance (NMR). spectroscopy and analysis of their characteristic enrichment patterns. The products of both pathways become, enriched when the enriched acetate is added to the fermentation of E. culmorum (HLX 1503). The extent of incorporation by either pathway depends on when the enriched precursors are added. The TCA cycle is more active earlier in the fermentation than the mevalonic acid pathway while later in the fermentation the mevalonic acid pathway is more active." The extent of ${ }^{13} \mathrm{C}$ incorporation in a mycotoxin is dependant upon the activity of the pathway that produces that particular

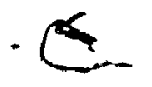


toxin. This means that as the-TCA cycle slows down the enrichment pattern in the products of this cycle, such as butenolide, changes. As the TCA cycle activity decreases, the activity of the mevalonic acid pathway increases and the label then appears preferentially in the products of this pathway. This coincides with the inset of secondary metabolism as discussed in the introduction. The shift in the metabolic pathways can be followed by the use ${ }^{13} \mathrm{C}$ NMR spectroscopy of the trude fungal' extracts. This tool can be used to detect specific metabolites in the complex fermentation mixture:

NMR Spectroscopy of Crude Fungal Extracts and some Possible Methods of Analyses

since ${ }^{13} \mathrm{C}$-acetate is added to the growth"medium, the NMR spectra of the crude fungal extracts allows the enriched products" to be observed: All of the carbon atoms that are not enriched will normally be at the level of the noise in the spectra unless the concentration of the unenriched metabolites is high. This property enables the extent of enrichment in other metabolites 'such as : the trichothecenes to be determined. In this study, the level of enrichment in 3-acetyldeoxynivalenol (3-ADON) was determined. (see 
appendix 1 for details) when the caricentration was high enough that the unlabelled carbon 3 (fig. 9) was visible in the spectrum. 3-ADON (3) is monitored as a sample trichothecene because it 'is' the most abundantly produced trichothecene of E. qumerum ( $H L X$ 1503).

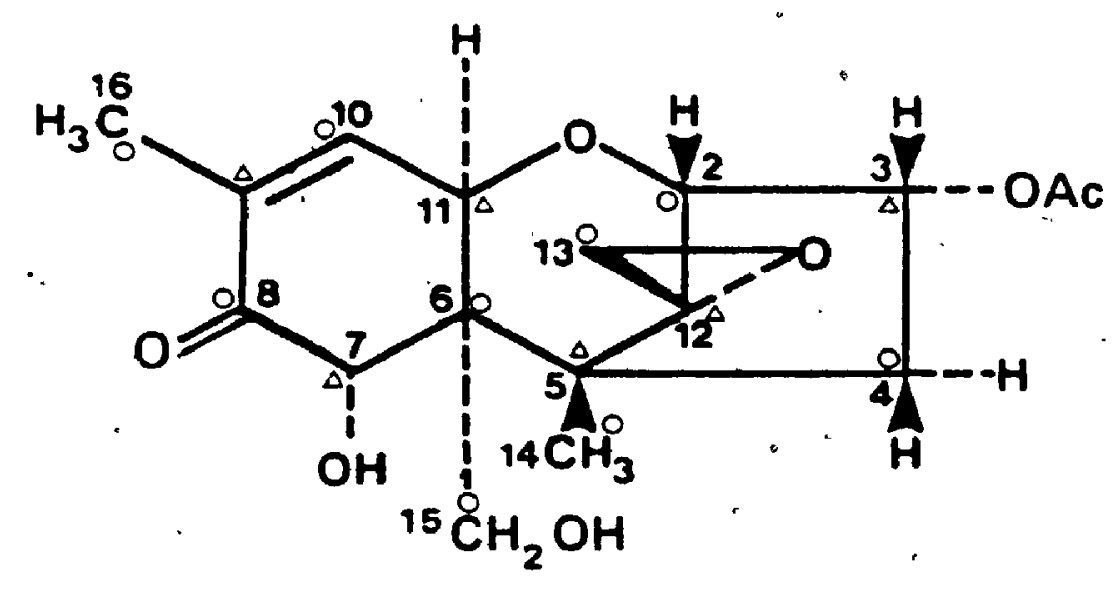

3

fig. 9 The structure and numbering system of 3-ADON (3). The carbon atoms arising from $13 \mathrm{C}-1$ acetate $(\Delta)$ and $13 \mathrm{C}-2$ acetate $(0)$ are indicated. 
Determination of Enrichment in Butenolide

Calculations to determine the extent of enrichment of butenolide (1) are a little more involved than those for 3-ADON and require the comparison of the sample spectrum to a natural abundance standard spectrum of butenolide (1). A direct comparison within the molecule, such as the comparison possible with 3-ADON, is not possible because ail of the non-carbonyl carbons of butenolide become enriched when ${ }^{13} \mathrm{C}-2$ acetate is added. The carbonyl carbons of butenolide from the enriched. sample spectrum and the natural abundance spectrum can be compared since all spectra are obtained with the same acquisition parameters.

* ${ }^{13} \mathrm{C}$ NMR Data \%f Butenolide

The ${ }^{13} \mathrm{C}$ NMR spectrum and stryeture of purem butenolide are shown in fig. $10_{4}$ The chemical shifts of each carbon atom are as follows: $\mathrm{C}-1$ occurs at $171.8 \mathrm{ppm}, \mathrm{C}-2124.1 \mathrm{ppm}, \mathrm{c}-3151.7 \mathrm{ppm}, \mathrm{C}-4.81 .9$ ppm, C-5 $171.6 \mathrm{ppm}$ and $\mathrm{C}-6$ occurs at $22.3 \mathrm{ppm}$. The acquisition parameters for this spectrum were the same as all spectra obtgined for the purpose of enrichment analysis from fermentation experiments. Carbon atoms 
- 2, 3,4, and 6 become enriched with ${ }^{13} \mathrm{C}-2$ acetate whereas carbons 1 and 5 become enriched when ${ }^{13} \mathrm{C}-1$ acetate is added to the fungal medium.

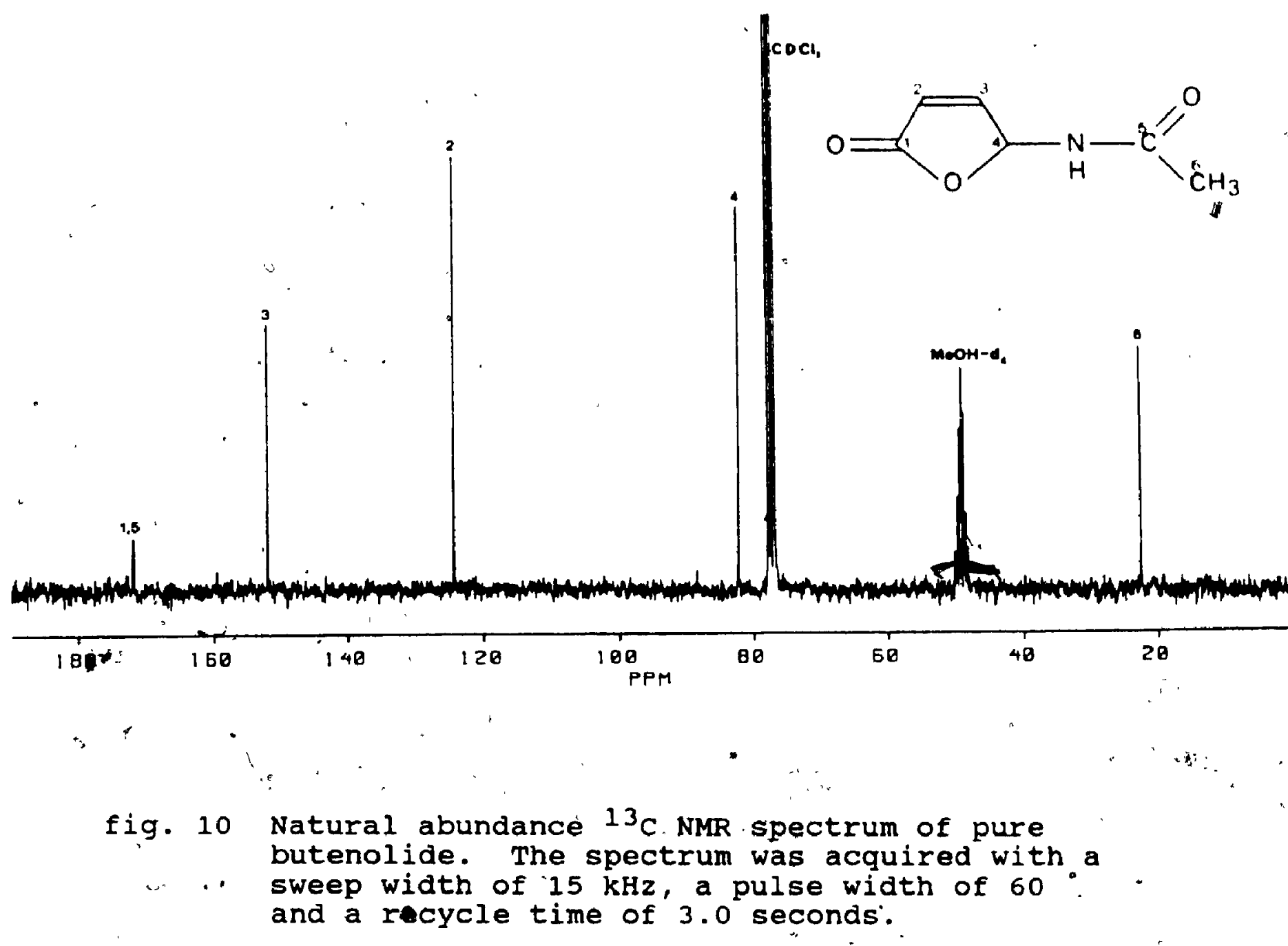


2-2 OPTIMIZATION OF. GROWIH CONDITIONS FOR MAXIMUM BUTENOLIDE PRODUCTION.

The Effect of Oxygenation on Butenolide Production

It was previously noted in our laboratory, during experiments to optimize the production of" trichothecenes, that increased oxygenation of the fermentation of $F$, culmorum HLX 1503 resulted in an increased production of butenolide (1). Thus, the conditions under which 3-ADON (3) production was maximized required energy would be put into the production of butenolide (1): The oxygenation conditions of the shake flask experiments were altered by using $25 \mathrm{~mL}$ and $50 \mathrm{~mL}$ of medium per flask. The $50 \mathrm{~mL}$ culture volume had been determined as the optimum, for the production Hhe trichothecenes. The lower volume of medium gives increased oxygenation provided the shaker speed remains constant.

Thirty flasks were inoculated; $15 \times 25 \mathrm{~mL}$ and $15 \times$ $50 \mathrm{~mL}$. The cultures were harvested after inoculation as follows: at $24 \mathrm{~h}, 90 \mathrm{~h}$, and $72 \mathrm{~h}, 3 \times 25 \mathrm{~mL}$ and. $3 . \mathrm{x}$ $50 \mathrm{~mL}$ cultures and at $30 \mathrm{~h}, 35 \mathrm{~h}$ and $48 \mathrm{~h}, 2 \times 25 \mathrm{~mL}$ and 2 × $50 \mathrm{~mL}$ cultures. Each culture was filtered, 
extracted and analyzed separately as described in section 3-1. The butenolide concentration within each harvest time was averaged and the standard deviation determined to give the final concentration at that time (table 1). The initial pH of the medium was 6.3 .

Table 1. The effect of oxygenation on the level of butenolide observed in cultures of $\mathrm{E}$. culmorum as indicated by $25 \mathrm{~mL}$ and $50 \mathrm{~mL}$ culture volumes.

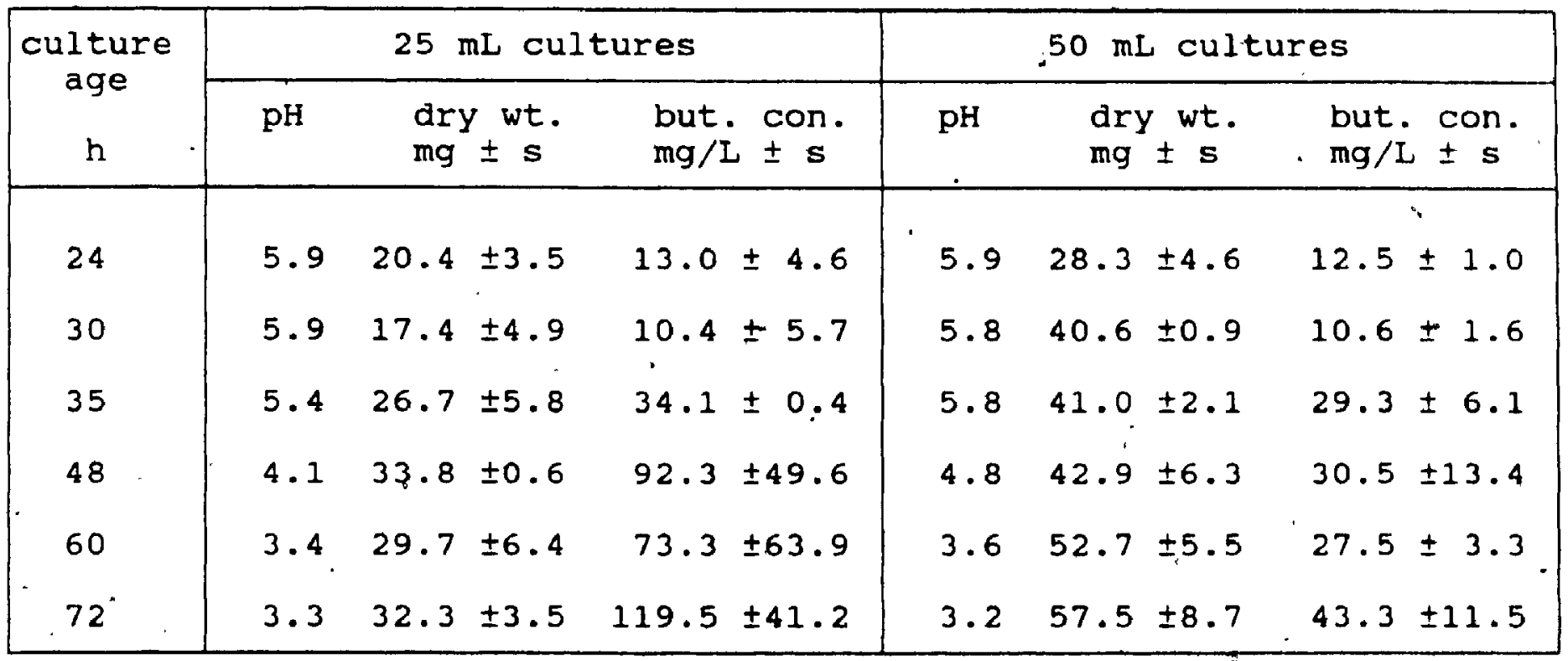

wt. - weight, but. - butenolide, con. - concentration, s - standard deviation.

It was discovered that although the cultures grown under higher oxygenation conditions $(25 \mathrm{~mL}$ culture volume) yielded higher concentrations of butenolidg the values were erratic and unreliable within a particular harvest (time (table 1). This effect was observed because the inoculum size was too small, therefore being irregular and creating variable cultures. The. culture conditions were, therefore, not reproducible 
even within an experiment. The inoculum irregularity produced cultures at varying stages of the fermentation.

The Significance of Dry Weight and $\mathrm{pH}$ Measurements.

The dry/weifht and $\mathrm{pH}$ are used as general indicators of the fulgal health in the shake flask experiments. The dry weight of a culture is the weight of the fungal mycelium after desiccation. In general, as the $\mathrm{pH}$ drops below about 3.5 the culture is in poor health and close to death even though the cultures can survive to a $\mathrm{pH}$ of as low as $2.0-2.5$ before death. The rate at which the $\mathrm{pH}$ drops is also indicative of the rate that the organism is growing. The reasonthat the $\mathrm{pH}$ drops in the medium is that the culture produces and excretes - acidic products. A rapid drop in the $\mathrm{pH}$ indicates that the fuldys is growing quickly and therefore producing more of these products.

An increase in the dry weight is typical of cells in balanced growth whereas a leveling off of the dry weight indicates that the cells have reached unbalanced growth and are no longer accumulating cell mass. A decrease in the dry weight indicates that the fungus is losing mycelial mass. Therefore, under the conditions 
in which dry weight decreases, cells are dying. A decrease in dry weight is typical of a dying culture.

The $25 \mathrm{~mL}$ cultures grow faster and die sooner than the $50 . \mathrm{mL}$ cultures as indicated by the more rapid drop in the $\mathrm{pH}$ (table. 1). Even though by $72 \mathrm{~h}$ the $\mathrm{pH}$ of both the $50 \mathrm{~mL}$ cultures and the $25 \mathrm{~mL}$ cultures is the same, the $25 \mathrm{~mL}$ cultures were growing more rapidly from $24 \mathrm{~h}$ to $48 \mathrm{~h}$, therefore, the quick drop in the $\mathrm{pH}$ is observed. The increase in the dry weight of each culture supports this concilusion. The dry weight of the $25 \mathrm{~mL}$ cultures reached a maximum at $48 \mathrm{~h}$ indicäting that the culture, was in balanced growth up to this time. After $48 \mathrm{~h}$ the cultures were in unbalanced growth as demonstrated by the constant dry weight. The $50 \mathrm{~mL}$ cultures, however, were accumulating mycelial mass up to $72 \mathrm{~h}$ (table 1). The dry weight of these cultures did not become constant indicating that they had not yet fully entered unbalancedgrowth. This may be one of the reasons why the butenolide and 3-ADON production in the $50 \mathrm{~mL}$ cultures was so much lower than the $25 \mathrm{~mL}$ cultures as production of secondary metabolites is highest during unbalanced growth.

The standard deviation of the butenolide concentration of the $25 \mathrm{~mL}$ cultures reaches a maximum of 63.9 at $60 \mathrm{~h}$ (table 1) whereas the nighest standard deviation of the $50 \mathrm{mr}$ cultures is 13.4 at $48 \mathrm{~h}$ 
(table 1). This gives some indication of the accuracy of the results of the $50 \mathrm{~mL}$ cultures as) compared with the $25 \mathrm{~mL}$ cultures. However, the highest concentration of butenolide, $119.5 \mathrm{mg} / \mathrm{L}$, by almost an order of magnitude, was obtained from the $25 \mathrm{~mL}$ cultures at $72 \mathrm{~h}$. The greatest concentration obtained with the 50 $\mathrm{mL}$ cultures was $43.3 \mathrm{mg} / \mathrm{L}$ which also occurred at $72 \mathrm{~h}$. The higher concentrations of butenolide in the $25 \mathrm{~mL}$ cultures, even with the high standard deviation, indicates that increased oxygenation is definitely a factor in increased butenolide (1) production.

Investigation of the observed Variability in $25 \mathrm{~mL}$ cultures

Similar results to those in table 1 , with respect to high butenolide concentrations, were observed when 21 flasks containing $25 \mathrm{~mL}$ of media were inoculated and cultivated. The experiment was repeated to investigate the variability of the values obtained previously. The cultures were harvested at $12 \mathrm{~h}, 24 \mathrm{~h}, 36 \mathrm{~h}, 48 \mathrm{~h}, 63$ $\mathrm{h}, 72 \mathrm{~h}$ and $86 \mathrm{~h},(3$ flasks per interval) and the filtrates were combined $(70 \mathrm{~mL})$. The combined viltrates were worked up and analyzed as described in section 3-1. The results (table 2) indiclete that the 
butenolide (1) production was high until $63 \mathrm{~h}$ and then it stopped.

Table 2. The concentration of butenolide found in $25 \mathrm{~mL}$ cultures of $\mathrm{E}$. culmerum HLX 1503 with respect to dry weight, $\mathrm{pH}$ and time.

\begin{tabular}{|c|c|c|c|}
\hline $\begin{array}{l}\text { culture } \\
\text { age }(h)\end{array}$ & pH & $\begin{array}{c}\text { dry weight } \\
\text { (mg) }\end{array}$ & $\begin{array}{l}\text { butenolide } \\
\text { conc. }(\mathrm{mg} / \mathrm{L})\end{array}$ \\
\hline 12 & $5 . \dot{9}$ & .42 .9 & 6.4 \\
\hline 24 & 5.3 & --- & 12.3 \\
\hline 36 & 5.2 & 84.0 & 23.5 \\
\hline 48 & 3.9 & 110.1 & 36.8 \\
\hline 63 & 3.2 & 141.6 & 69.3 \\
\hline 72 & 3.4 & 120.2 & 54.8 \\
\hline 86 & 3.4 & 109.5 & 66.8 \\
\hline
\end{tabular}

After $63 \mathrm{~h}$ (table 2 ), the tungal weight dropped, the $\mathrm{pH}$ remained low and the butenolide (1) concentration remained approximately constant which are all -indications that the cultures were dying or already dead. The butenolịde (1) concentration reached a maximum of $69.3 \mathrm{mg} / \mathrm{L}$ at $63 \mathrm{~h}$. Since the dry weight dropped quite significantly after $63 \mathrm{~h}$ and the butenolide concentration did not change substantially, it can be. concluded, that the cultures had died. The slight rise in the $\mathrm{pH}$ of the medium is likely due to the degrading dead cells which release the contents of 
the cellis as they degrade. The cell contents is at a $\mathrm{pH}$ of about 6.5 and as more cells lyse the $\mathrm{pH}$ increases slightly.

This experiment confirmed the fact that the $25^{\circ} \mathrm{mL}$ cultures grow at a more rapid rate than do $50 \mathrm{~mL}$ - cultures and that they die earlier. It can therefore, be concluded that the higher level of oxygenation of the $25 \mathrm{~mL}$ cultures caused them to enter unbalanced growth earlier than the $50 \mathrm{~mL}$ cultures due to faster utilization of the nutrient sourcies.

\section{2-3 OPTIMIZATION OF LABELLING CONDITIONS.}

The conditions for the incorporation of enriched precursors into the trichothecenes have been optimised for $E$ e culmorum HLX 1503 (20). As either the mevalonic acid pathway or the TCA cycle function, there is a greater demand for precursors of those pathways. Since pcetate is a universal precursor for many/of the metabolic pathways in the cell, it is necessary to.add it when the pathway of interest is the most active. Therefore, in order to, optimize the incorporation of the label into butenolide, it was necessary to add the enriched acetate to the medium while the TCA cycle was still relatively active and at the onset of butenolide 
production. In this way, the label will preferentially be used in the production of butenolide (1) through the TCA cycle rather than for the production of the trichothecenes through the mevalonic acid cycle. The a mevalonic acia cycle becomes more active in the. production of the trichothecenes as the fungus enters unbalanced growth. The TCA cycle and the production of butenolide (1) are high at the end of balanced growth as the nitrogen source becomes limited.

Incorporation of ${ }^{13} \mathrm{C}$ Acetate into Butenolide"

In order to optimize the enrichment conditions, the incorporation of the enriched precursors into 2 butenolide must be studied. A shake flask experiment. was performed, as described in section 3-1, to monitor the dynamics of the incorporation of ${ }^{13} \mathrm{C}$ enriched acetate into metabolites of Ee culmorum $\mathrm{HLX} 1503^{\circ}$ by ${ }^{13} \mathrm{C}$ NMR.

il $\mathrm{C}$-enriched acetate was added, over a $24 \mathrm{~h}$ period, to 18 flasks containing $25 \mathrm{~mL}$ of inoculated media. The filtrates "of triplicate cultures of each of three acetate species, ${ }^{3} \mathrm{C}-1$ acetate, $13 \mathrm{c}-2$ acetate and, as a control, natural abundance sodium acetate, were combined and analyzed. All acetate,precursors were added to the culture flasks to a final concentration of 
. $1 \mathrm{mg} / \mathrm{mL}$ of medium and were added in 5 equal aliquots ' from $12 \mathrm{~h}$ to $36 \mathrm{~h}$ at equal time intervals. The label is added over a 24 hour period so as not to disturb the $\mathrm{pH}$ of the medium too much since culture $\mathrm{pH}$ seems to be a critical factor in the metabolism of the fungus. In this way, shock to the fungal system is prevented and the metabolism is disrupted as little as possible.

Half of the experiment ( 9 flasks) was harvested after $48 \mathrm{~h}$ (or $12 \mathrm{~h}$ after, the final aliquot of label was added) and the rest of the cultures were harvested at $72 \mathrm{~h}$.

There was a higher concentration of butenolide in all samples harvested at, $7.2 \mathrm{~h}$ thain those harvested at $48 \mathrm{~h}$ (table 3 ). The pH renained high throughout the experiment because the sodium acetate solutions added are slightly basic. 
Table 3. Butenolide concentrations and pH values in shake flask cultures of $F$. culmerum HLX 1503 fed enriched and unenriched acetate and harvested at $48 \mathrm{~h}$ and' $72 \mathrm{~h}$.

\begin{tabular}{|l|c|c|c|}
\hline $\begin{array}{l}\text { species } \\
\text { added }\end{array}$ & $\begin{array}{c}\text { time } \\
\text { harvested }\end{array}$ & $\mathrm{pH}$ & $\begin{array}{c}\text { butenolide } \\
\text { conc. mg/L }\end{array}$ \\
$\begin{array}{l}\text { unenriched } \\
\text { acetate }\end{array}$ & 48 & 6.4 & 6.4 \\
$\begin{array}{l}13 \text { C-1 } \\
\text { acetate }\end{array}$ & 42 & 6.1 & 37.5 \\
13 C-2 & 72 & 6.4 & 9.3 \\
acetate & 48 & 6.1 & 10.3 \\
\hline
\end{tabular}

conc. - concentration.

The variance in the: butenolide concentration for each of the chemical species added to the cultures can be explained in terms of the relationship secondary metabolite formation seems to have with $\mathrm{pH}$. The low concentration of butenolide observed in the cultures fed ${ }^{13} \mathrm{C}-1$ acetate (table 3) occurred because the $\mathrm{pH}$ remained above 6.3 for both harvest times in these cultures. From the data for the cultures of unenriched acetate (table 3), concentration of butenolide was much higher at $72 \mathrm{~h}$ than it was at $48 \mathrm{~h}$ and the $\mathrm{pH}$ dropped to 6.1. For the cultures enriched with ${ }^{13} \mathrm{C}-2$ acetate the $\mathrm{pH}$ was below 6.2 at al! times and the production of butenolide (1) was higher than any of the cultures where the $\mathrm{pH}$ was above 6.3 , independent of the 
species added. This may be an indication that the metabolism of butenolide may be $\mathrm{pH}$ dependent:

The difference in $\mathrm{pH}$ between enriched ${ }^{13} \mathrm{C}$ acetate species is likely due to the purity of the initial compounds. All precursors are used without further purification from the manufacturer. The production of butenolide and 3-ADON was very slow until the $\mathrm{pH}$ dropped below what seems to be a critical level at about $\mathrm{pH}$ 6.3. This is also likely to be one of the factors influencing the end of balanced growth and the initiation of secondary metabolite production in unbalanced growth. 1

The ${ }^{13} \mathrm{C}$ NMR spectra (fig. 11) show that both labels were incorporated into butenolide. There were no significant differences in enrichment characteristics with the same precursor between the cultures harvested at $48 \mathrm{~h}$ and those harvested at $72 \mathrm{~h}$. Therefore, the $72 \mathrm{~h}$ spectra are used as representative of this experiment.

Note that cultures fed ${ }^{13} \mathrm{C}-2$ acetate show enrichment in 4 carbon atoms $(\mathrm{fig} .11 \mathrm{~B}), \cdot 3$ of those carbons being adjacent and giving characteristic ${ }^{13} \mathrm{C}-{ }^{13} \mathrm{C}$ splitting patterns. All of these carbon atoms are $\mathrm{CH}$ or $\mathrm{CH}_{3}$

1 All subsequent shake flask experiments were performed with $5.0 \mathrm{~mL}$ cultures rather than $25 \mathrm{~mL}$. cultures to keep the results as consistent as possible and to minimize inoculum irregularities. 
carbons. The two carbonyl carbons in butenolide (1) arise from ${ }^{13} \mathrm{C}-1$ acetate (fig. $11 \mathrm{~A}$ ). Both of the results are consistent with the expected enrichment pattern from TCA cycle products (fig. 6 and 7 ) as described in the introduction. 
fig. $11{ }^{13} \mathrm{C}$ NMR spectra showing the incorporation of

(A) ${ }^{13} \mathrm{C}-1$ acetate and $B$ ) ${ }^{13} \mathrm{C}-2$ acetate into

- butenolide (1). The numbers correspond to the carbon atoms of butenolide. A denotes peaks from 3-ADON; S solvent, $\mathrm{CDCl}_{3}$.

a. 


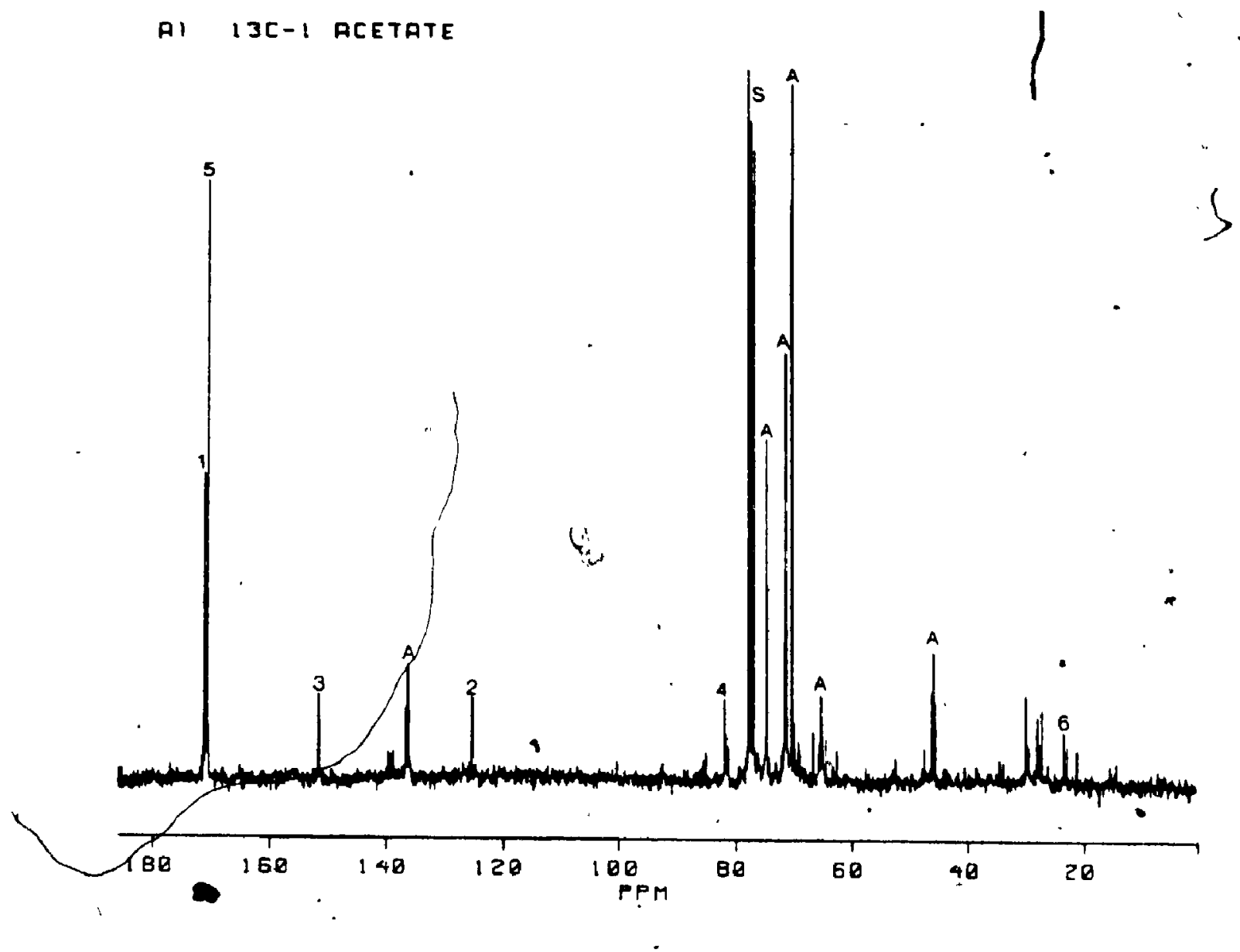

B) I3C-2 ACEJATE

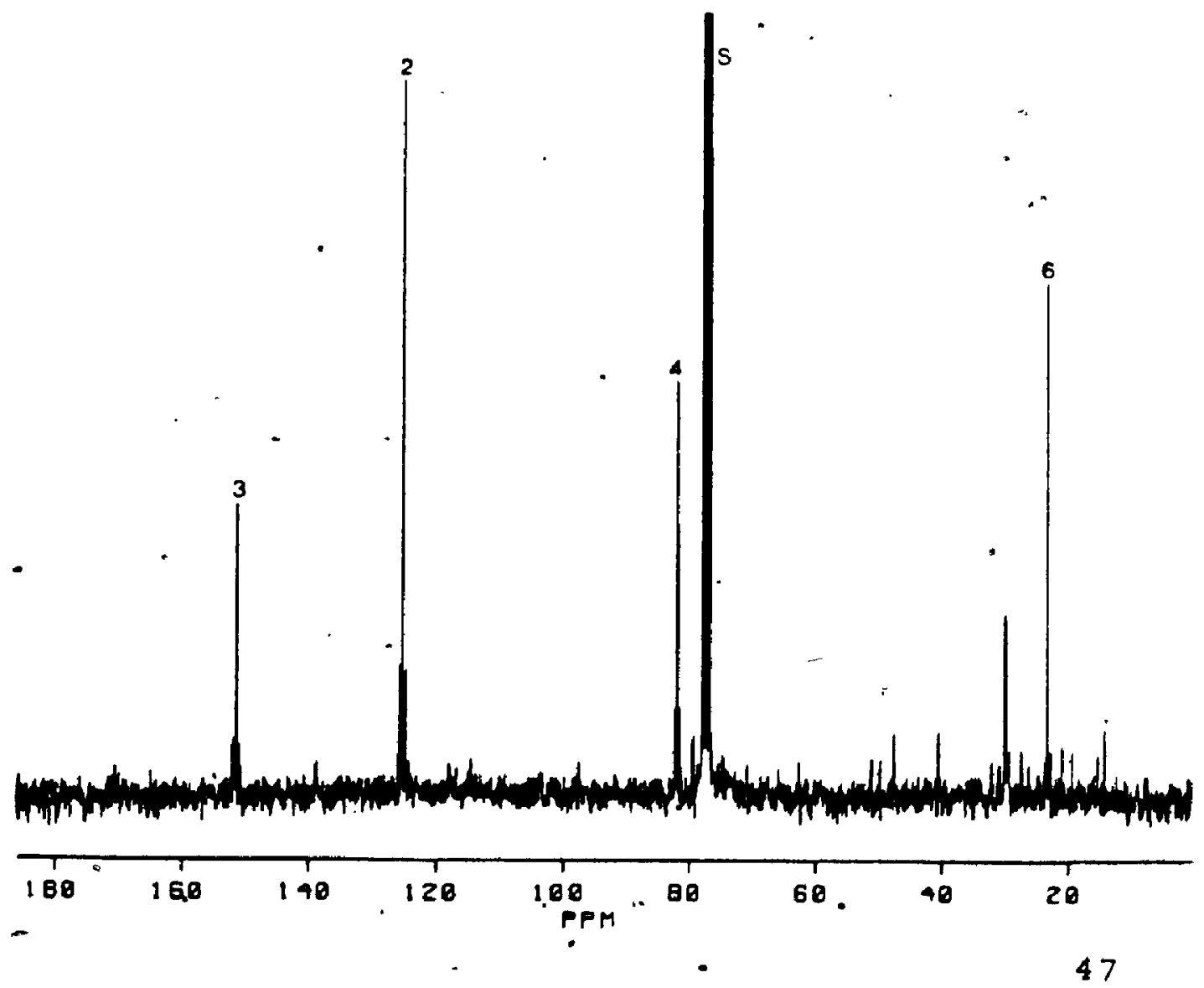


Maximization of Incorporation of stable Isotope Precursors into Butenolide

since this study was an elucidation of the biosynthetic origin of butenolide using stable isotopes, it was necessary to maximize the -incorporation of the enriched precursors. This was accomplished" by adding enriched acetate to the fermentation of shake flask cultures at various times and harvesting the cultures at specific times after the label was completely added. The crude fungal extracts were then analyzed by ${ }^{13} \mathrm{c}$ NMR to determine the extent of enrichment in both butenolide (1) and 3-ADON (3) as described in section 3-1a. The trichothecene; 3-ADON (3), was analyzed because we sought to minimize the extent of enrichment in this class of compounds and 3-ADON is the most abundant trichothecene produced by this species of Eusarium.

In each of the five simultaneous experiments, both ${ }^{13} \mathrm{C}-1$ and ${ }^{13} \mathrm{C}-2$ acetate were added to three separate flasks respectively, Natural abundance sodium acetate was added to three cultures in two of the five experiments, to give representative controls. There were a total of 36 cultures used for this experiment. Labelling was started at $12 \mathrm{~h}, 36 \mathrm{~h}, 48 \mathrm{~h}$ and $60 \mathrm{~h}$ and 
harvested $24 \mathrm{~h}$ after the label was completely added. One experiment, in which label was added at $36 \mathrm{~h}$, was harvested $109 \mathrm{~h}$ (at $169 \mathrm{~h}$ ) after the label was completely added. This experiment was performed to determine the effect of longer culture time on the level of incorporation of the label. As before, the label was added in 5-equal aliquots over a $24 \mathrm{~h}$ period. All final concentrations for the acetates were $1 \mathrm{mg} / \mathrm{mL}$ of medium.

Table 4. The butenolide and 3-ADON concentrations of $F$, culmerum HLX 1503 cultures fed acetate and harvested at different times throughout the fermentation.

\begin{tabular}{|c|c|c|c|c|}
\hline $\begin{array}{l}\text { acetate } \\
\text { species }\end{array}$ & $\begin{array}{l}\quad \text { time } \\
\text { label } \\
\text { added }\end{array}$ & $\begin{array}{l}\text { (h) } \\
\text { harvest }\end{array}$ & $\begin{array}{c}\text { butenolide } \\
\text { concentration } \\
\mathrm{mg} / \mathrm{L}\end{array}$ & $\begin{array}{c}\text { 3-ADON } \\
\text { concentration } \\
\mathrm{mg} / \mathrm{L}\end{array}$ \\
\hline${ }^{13} c-1$ & 12 & 60 & 44.35 & 25.36 \\
\hline${ }^{13} c-2$ & & & 80.69 & 34.18 \\
\hline${ }^{13} c-1$ & 36 & 84 & 152.26 & 88.61 \\
\hline${ }^{13} c-2$ & & & 99.71 & 58.56 \\
\hline unenriched & & & 90.56 & 49.57 \\
\hline${ }^{13} c-1$ & 36 & 169 & 75.76 & 138.13 \\
\hline${ }^{13} c-2$ & & & 107.44 & 176.84 \\
\hline $13 \mathrm{C}-1.7$ & 48 & 96 & 99.19 & 88.50 \\
\hline $13 c-2$ & & & 148.23 & 163.92 \\
\hline unenriched & & & 96.01 & 64.93 \\
\hline${ }^{13} c-1$ & 60 & 108 & 94.43 & 104.36 \\
\hline${ }^{13} c-2$ & & & 147.92 & 95.56 \\
\hline
\end{tabular}


The concentrations of butenolide (1) and 3-ADON (3) along with the label addition and harvest times are shown in table 4. Triplicate cultures were harvested and the filtrates combined and analyzed as one sample as described in section 3-1. Notice that although the later harvest times may have higher concentrations of butenolide, the earlier harvest times have the desired lower relative 3-ADON concentrations.

The ${ }^{13} \mathrm{C}-\mathrm{NMR}$ data (fig. 12) from this experiment indicated that the optimum time for adding enriched acetate to obtain the highest incorporation into butenolide and ${ }_{G}$ the least incorporation into the trichothecenes was $12 \mathrm{~h}$. All of the times after $12 \mathrm{~h}$ have too much incorporation in 3-ADON. Earlier work performed while adding the enriched acetate at $24 \mathrm{~h}$ gave favorable results as well (fig. 11) with higher incorporation into butenolide than 3-ADON. When the cultures were harvested at $169 \mathrm{~h}$ after adding the label at $36 \mathrm{~h}$ there was higher incorporation into 3-ADON relative to butenolide than when the cultures were harvested at $84 \mathrm{~h}$ as indicated by the spectra shown in fig. 13. This is likely a factor of both metabolic activity (ie high incorporation through the mevalonic acid pathway as opposed to the TCA cycle) and the higher concentration of 3-ADON which makes this 
molecule more visible by NMR. The lower level of incorporation of ${ }^{13} \mathrm{C}$ acetate into $3-A D O N$ in the cultures 'härvested at $84 \mathrm{~h}$ occurred because the enriched acetate was only available for incorporation for the first $24 \mathrm{~h}$ after the final addition to. the medium. Although the precursor was added at a time when the mevalonic acid cycle activity was increasing and the TCA cycle activity was decreasing (as indicated by the high level of incorporation in 3-ADON at $169 \mathrm{~h}$ and toxin production profiles from previous experiments) the cultures were harvested before trichothecene production was greater than the total amount of butenolide produced. The concentration of 3-ADON was much higher in the $169 \mathrm{~h}$ sample than the $84 \mathrm{~h}$ sample whereas the butenolide concentration remained the same indicating that the mevalonic acid cycle was more active than the TCA cycle. 


fig. $12{ }^{13} \mathrm{C}$ NMR spectra of the crude fungal extract
from $50 \mathrm{~mL}$ shake flask cultures of $E$ cylmorum
HLX 1503 to which ${ }^{13} \mathrm{C}-2$ acetate was added at
sevéral times. Label times and harvest times
are printed on the spectra. B butenolide; A
3-ADON; solvent, $\mathrm{CDCl}_{3} \cdot$



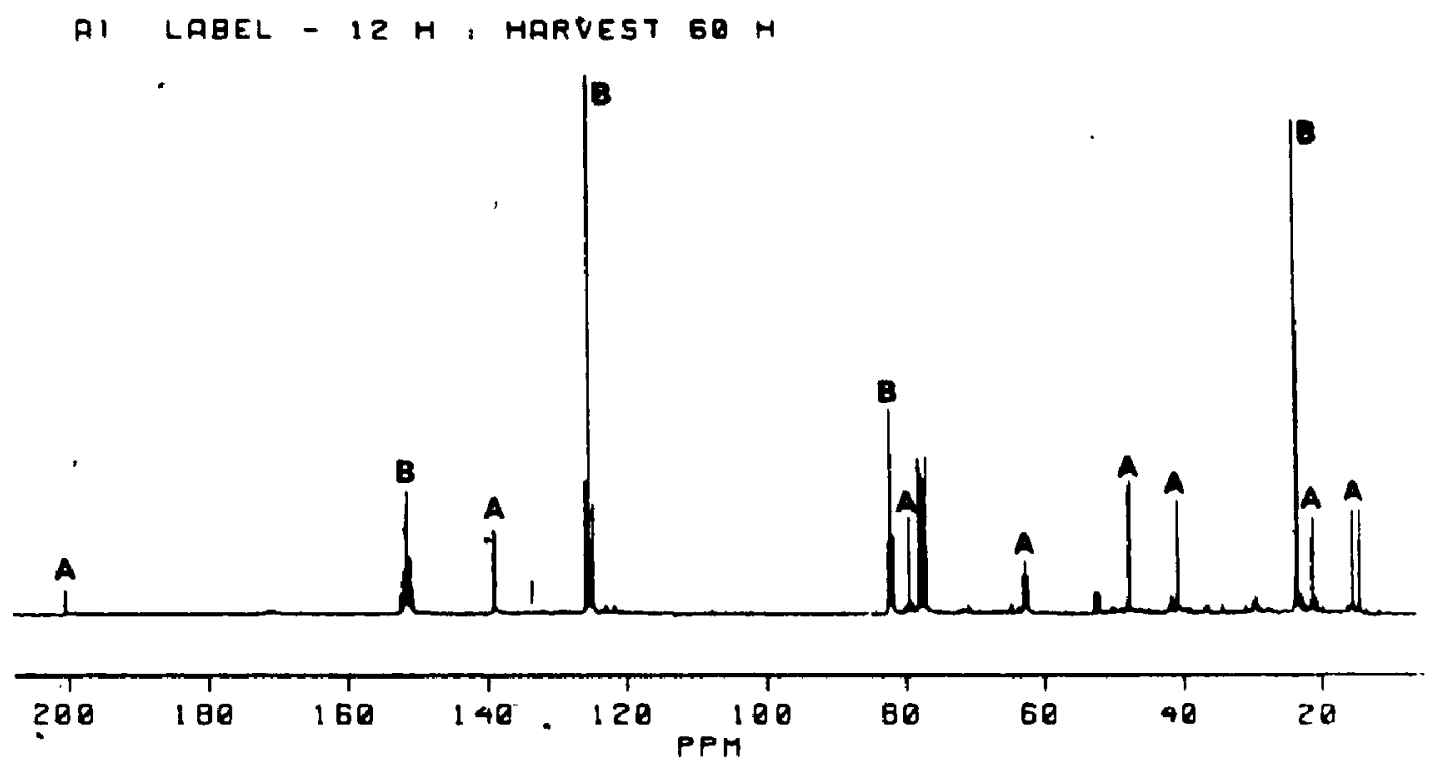

Bi LABEL - 36 H: HARVEST - $94 \mathrm{H}$

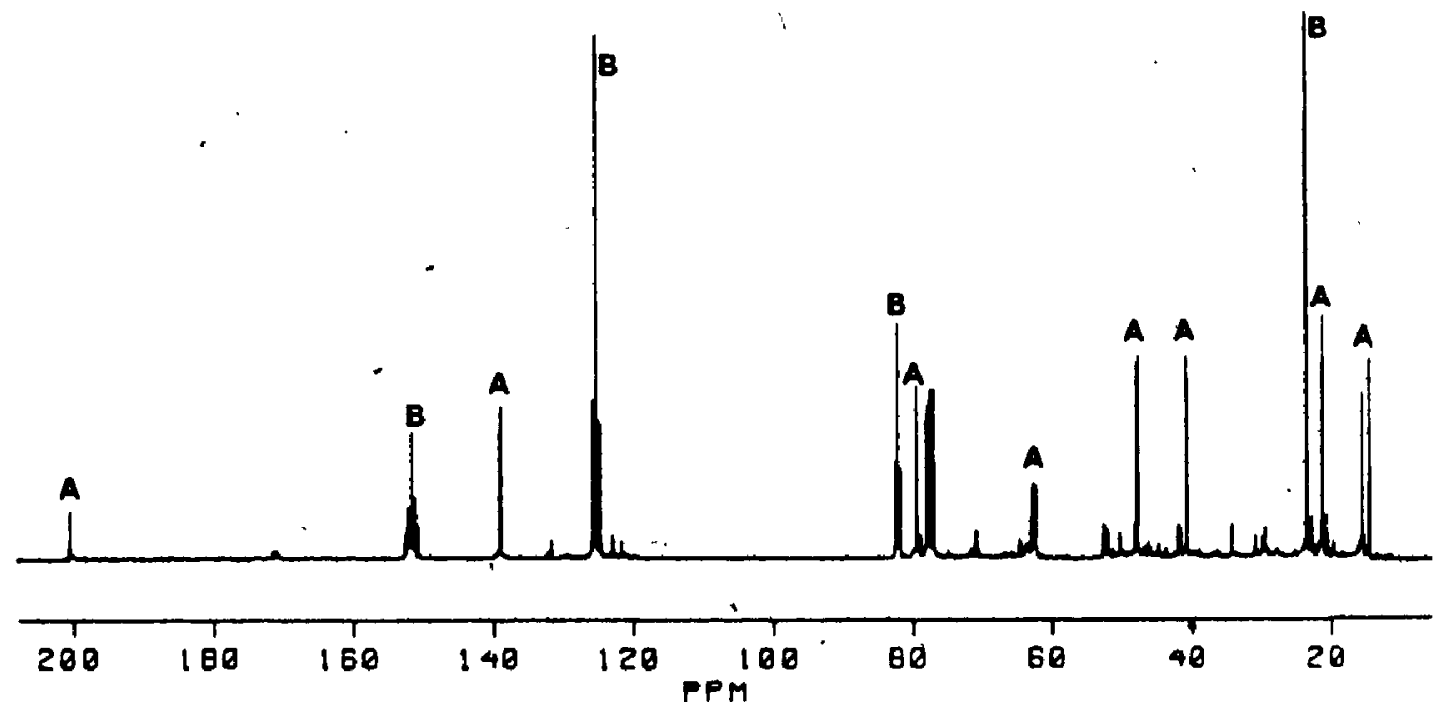

CI LABEL - 4B H, HARVEST - $96 \mathrm{H}$

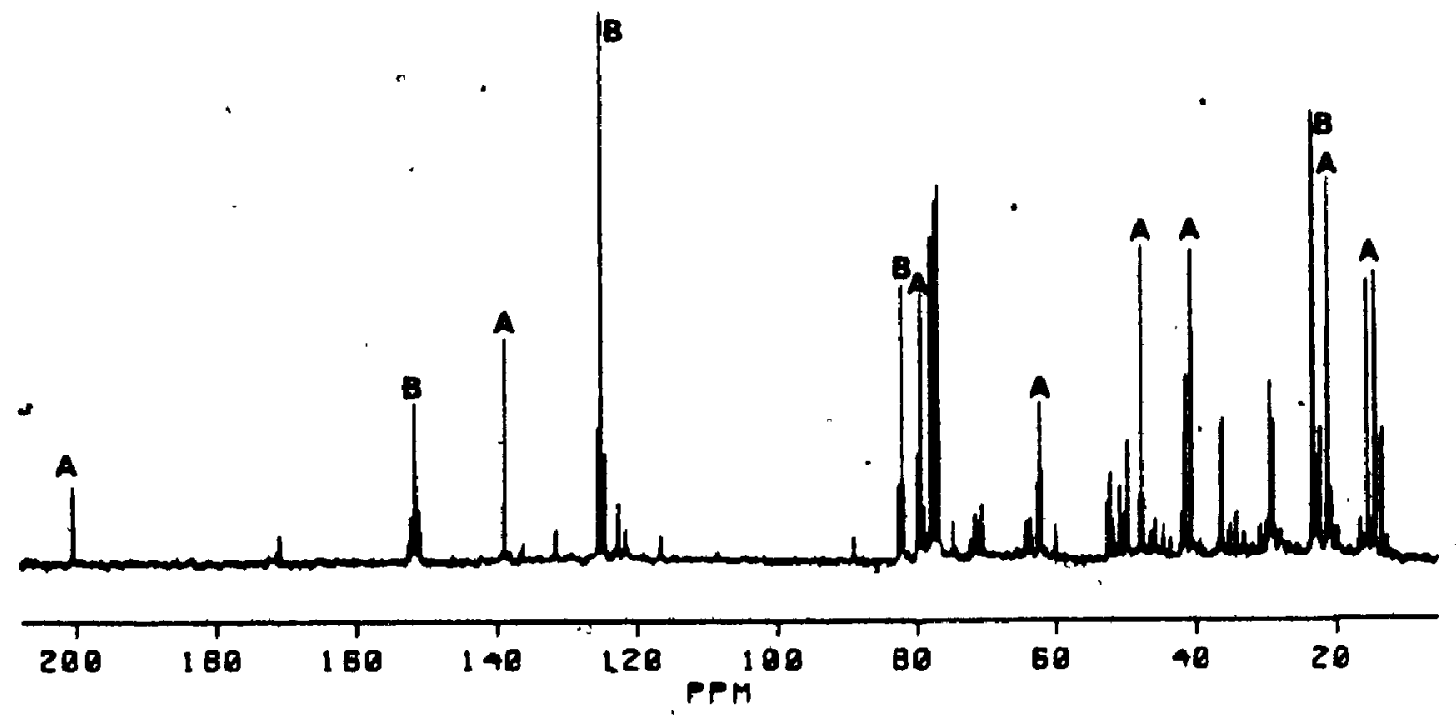



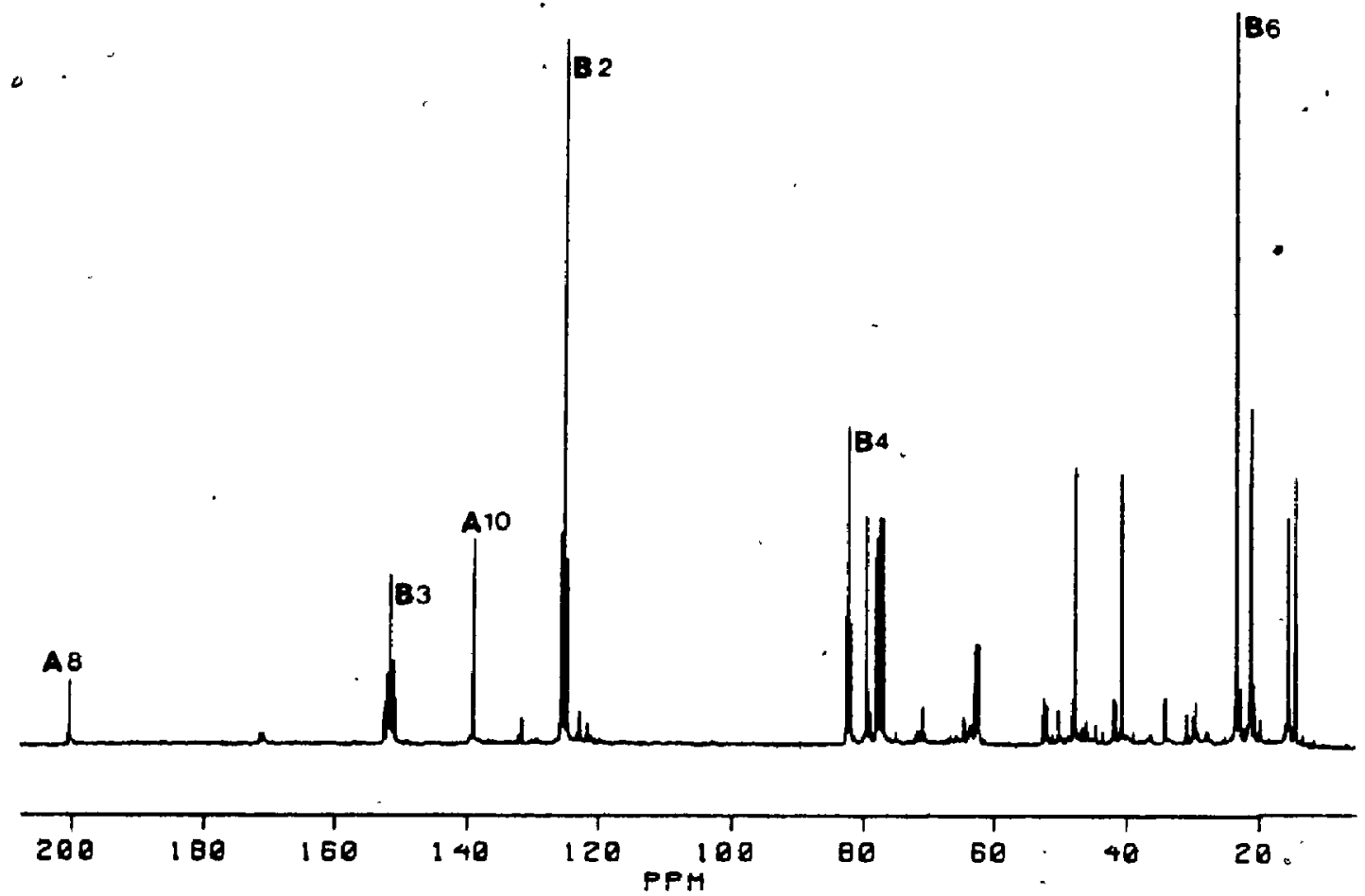

B) LABEL - 36 H: HRRVEST - $169 \mathrm{H}$

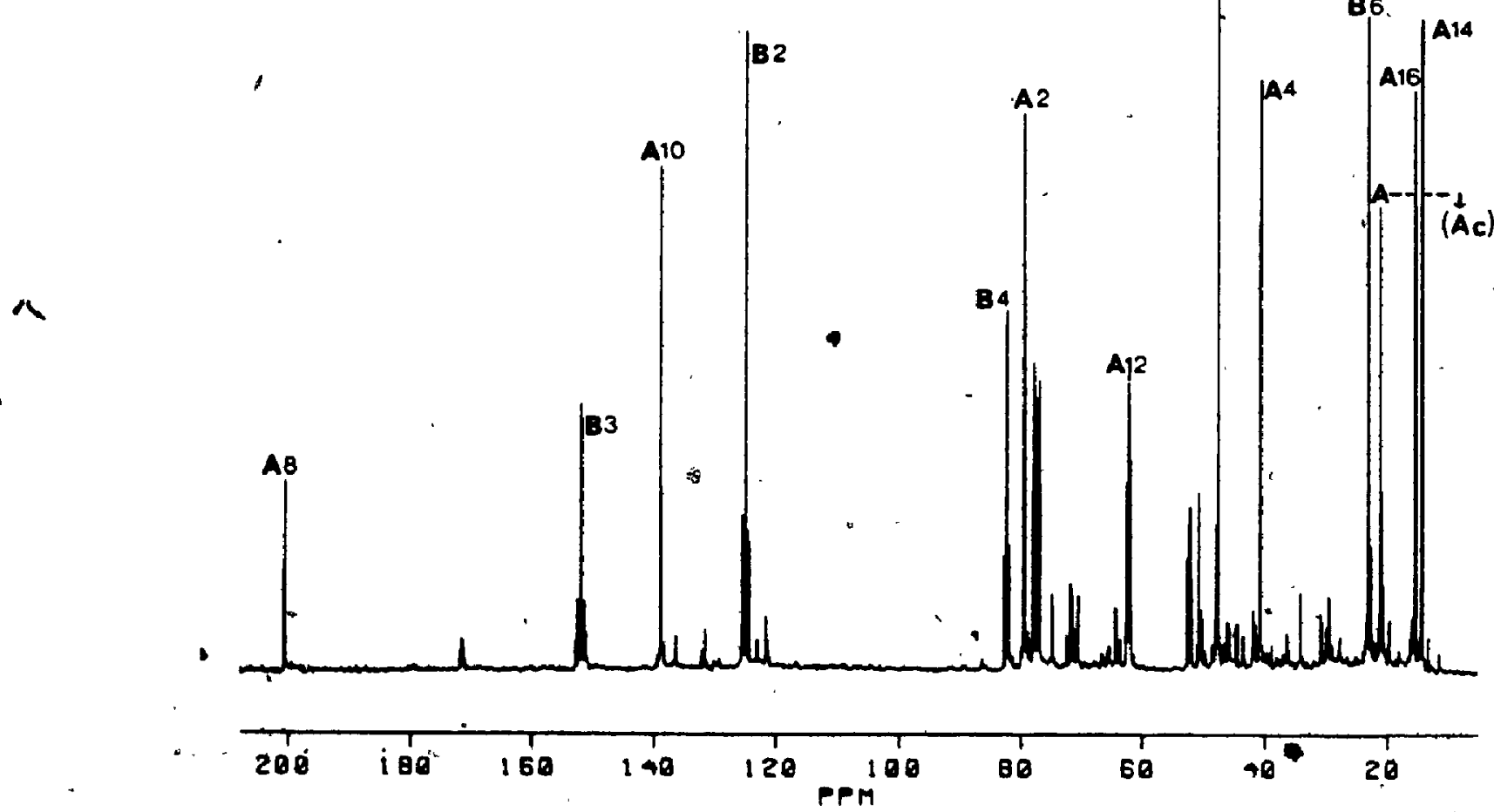

fig. $13{ }^{13} \mathrm{C}$ NMR spectra showing the effect of harvest time on the incorporation of $13 \mathrm{C}-2$ acetate into butenolide $(\underline{1})$ and $3-A D O N(3)$. A 3-ADDON; $B$ butenolide. The numbers indicate which. carbon is observed. 
2-4 IS GLUTAMIC ACID A METABOLIC PRECURSOR OF BUTENOLIDE?

From an analysis of, the structures of butenolide. (1) and glutamic acid (2), we suggested that glutamic acid may be a possible metabolic precursor of butenolide.

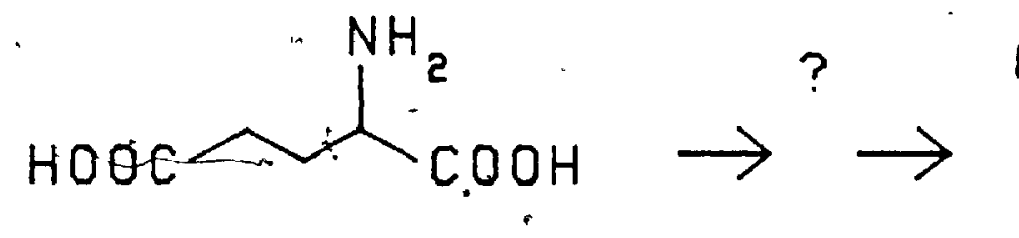

Glytamic acid
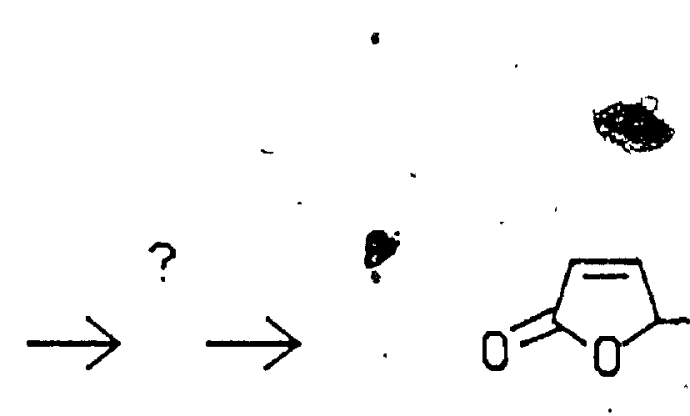<smiles>CC(=O)NC1C=CC(=O)O1</smiles>

Butenolide

fig. 14. Is glutamic acid a precursor for butenolide biosynthesis?

This would explain the presence of the nitrogen contained in the molecule and give a possible starting point for the cyclization to the lactone. Beyond this a simple decarboxylation, desatúration and $N$ 
acetylation would yield the final product, butenolide (1).

Glutamic acid (2) was determined as a precursor of butenolide by adding both ${ }^{13} \mathrm{C}-5$ glutamic acid and ${ }^{15} \mathrm{~N}$ glutamic acid to each" of 3 shake flask cultures at a final concentration of $20 \mathrm{mg} / \mathrm{flask}$ (i.e. $10 \mathrm{mg}$ of each enriched amino acid). Natural abundance glutamic acid (2) was also added to 3 cultures to a final concentration of $20 \mathrm{mg} /$ flask. 'Suitable controls with no species added and containing $13 \mathrm{C}-1$ acetate with a final concentration of $1 \mathrm{mg} / \mathrm{mL}$ of medium, were also run with 3 cultures of each. The labels were added from $26 \mathrm{~h}$ to $52 \mathrm{~h}$ in 5 equal aliquots and all cultures were harvested at $74 \mathrm{~h}$. The 3 filtrates of each replicate were comblhed, worked up and analyzed as one sample as described in sections, $3-1$ and $3-1 a$.

The cultures to which glutamic acid (2) was added produced much higher concentrations of butenolide (table 5) than the control cultures. This, alone, was a good indication that glutamic acid ( $\underline{\dot{L}})$ is a precursor of butênolide (1). 
Table 5. The effect of adding glutamic acid to cultures of $F_{\text {. }}$ culmerum HLX 1503 on the butenolide and 3-ADON concentrations.

\begin{tabular}{|l|c|c|c|}
\hline sample & $\mathrm{pH}$ & $\begin{array}{c}\text { butenolide } \\
\text { conc. mg/L }\end{array}$ & $\begin{array}{c}\text { 3-ADON } \\
\text { conc. mg/L }\end{array}$ \\
\hline $\begin{array}{l}\text { control } \\
13 \mathrm{C}-1 \text { AC }\end{array}$ & 2.9 & 70.7 & 18.8 \\
$\begin{array}{l}\text { unenriched } \\
\text { glutamate } \\
13 \text { C-5/15N } \\
\text { glutamate }\end{array}$ & 2.5 & 23.8 & 14.0 \\
\hline
\end{tabular}

AC - acetate.

The $\mathrm{pH}$ of the medium was slightly decreased by the addition of the glutamic acid (table 5). This slight decrease in the $\mathrm{pH}$ at a $\mathrm{pH}$ of 2.5 , the final observed $\mathrm{pH}$, has little effect on the toxin metabolism, however, a decrease in $\mathrm{pH}$ earlier in the fermentation may cause the cultures to enter unbalanced growth early. The 3-ADON (3) level was higher in the two cultures to which glutamic acid (2) had been added which indicates that the secondary metabolism was stimulated. The stimulation may be through the effect on the pH earlier in the fermentation which would alter the growth curve of the culture or it may be due to the glutamic acid (2) itself. The effect of glutamic acid on the cultures was further investigated in subsequent experiments. 
The enriched çarbon from the ${ }^{13} \mathrm{C}-5$ glutamic acid was incorporated into butenolide (fig. 15). These spectra show that one of the carbonyl carboris in butenolide (1). is ${ }^{13} \mathrm{C}$ enriched. The enriched carbon is $\mathrm{C}-1$ of butenol'ide and arises from the ${ }^{13} \mathrm{C}-5 \cdot$ glutamic acid. This indicates that glutamic acid (2) is a precursgr of. butenolide (1).

This experiment was repeated to produce more ${ }^{13} \mathrm{c} /{ }^{15} \mathrm{~N}$ enriched butenolide.' The concentration of glutamic acid (2) added was raised to $30 \mathrm{mg} / \mathrm{flask}$, added. from $24 \mathrm{~h}$ to $48^{\mathrm{h}} \mathrm{h}$ and harvested at $72 \mathrm{~h}$. The change in glutamic acid concentration resulted in a reduction in the yield of butenolide from the $211.7 \mathrm{mg} / \mathrm{L}$ obtained id the previous experiment to $61.7 \mathrm{mg} / \mathrm{L}$. The $3-A D O N$ (3) concentration was 21.7 .m. The reduction in the yield of butenolide, relative to the first glutamic acid experiment, with the increased glutamic acid added indicates that this increase has an effect on the metabolws of the fungus. The butenolide production, relative to the controls, was unaffected with the increased glutamic acid whereas in the previous experiment the toxin production had been stimulated. 

P) UNLABELLED GLUTAMIC ACID

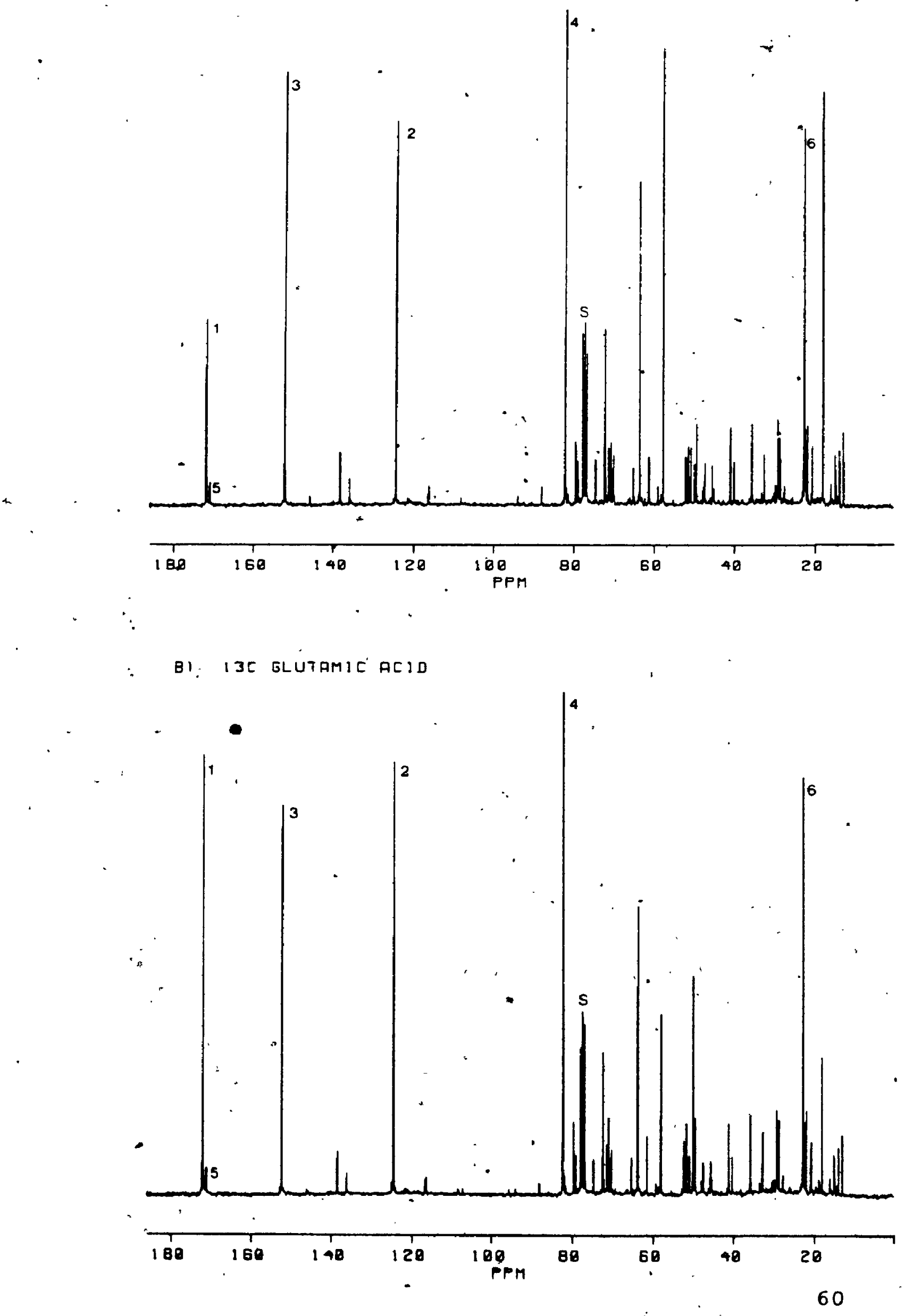


-A third experiment using a concentration of $20 \mathrm{mg}$ of amino acid per flask resulted in a slightly nigher butenolide concentration of $106.7 \mathrm{mg} / \mathrm{L}$. The 3 -ADON (3) concentration for this experiment was $50.1 \mathrm{mg} / \mathrm{L}$. The data for these three experiments are summarized in table 6 .

Table 6. The effect of glutamic acid concentration on the production of butenolide and 3-ADON in shake flask cultures of $E_{\text {e culmorum HLX } 1503 .}$

\begin{tabular}{|c|c|c|}
\hline $\begin{array}{c}13 \mathrm{C} /{ }^{15} \mathrm{~N} \text { glutamic } \\
\text { acid added } \\
\text { mg/flask }\end{array}$ & $\begin{array}{c}\text { butenolide } \\
\text { conc. } \\
\mathrm{mg} / \mathrm{L}\end{array}$ & $\begin{array}{c}3 \text {-ADON } \\
\text { conc. } \\
\mathrm{mg} / \mathrm{L}\end{array}$ \\
\hline 20.0 & 211.9 & 44.0 \\
30.0 & 61.7 & 21.7 \\
20.0 & 106.7 & 50.1 \\
\hline
\end{tabular}

The addition of glutamic acid (2) to the medium of shake flask "cultures increased the butenolide (1) production provided the concentration was not too high. yot only was an increase in butenolide production observed but also an increase in the 3-ADON production. Therefore, the metabolism of the cells seems to be stimulated with the addition of the glutamic acid (2). This stimulation is only experienced if the concentration of glutamic acid not higher than 20 
mg/flask. At $30 \mathrm{mg} / \mathrm{flask}$, the metabolism of the fungus" seems tó be unaffected.

The source of the nitrogen in butenolide was determined to be glutamic acid through the use of $15 \mathrm{~N}$ glutamic acid and ${ }^{15} \mathrm{~N}$ NMR spectroscopy. INEPT (27) was used in recording the spectrum to overcome the problems of the negative noe's and the long relaxation times $\left(T_{1}\right)$ of the ${ }^{15} \mathrm{~N}$ nucleus $(28)$. The ${ }^{15} \mathrm{~N}$ spectrum of the crude fungal extract of $E_{\text {e }}$ culmorum HLX 1503 to which ${ }^{13} \mathrm{C}-5$ glutamic acid and ${ }^{15} \mathrm{~N}$ glutamic acid were added is shown in figure 16. By comparison of this spectrum with the spectrum of the natural abundance butenolide, it was determined that the resonance observed at -257 ppm was due to butenolide: The ${ }^{15} \mathrm{~N}$ resonance-for glutamic acid occurs at -332 ppm. Resonances are determined with respect to neat nitromethane. The incorporation of the ${ }^{15} \mathrm{~N}$ from glutamic acid was only observed in butenolide which, again, is a good indication that glutamic acid is likely a precursor of butenolide. The ${ }^{13} \mathrm{C}$ NMR spectrum of the same extract also showed incorporation of the ${ }^{13} \mathrm{C}-5$ glutamic acid into butenolide and no other enriched resonances (ie. ${ }^{13} \mathrm{C}-5$ glutamic acid). 


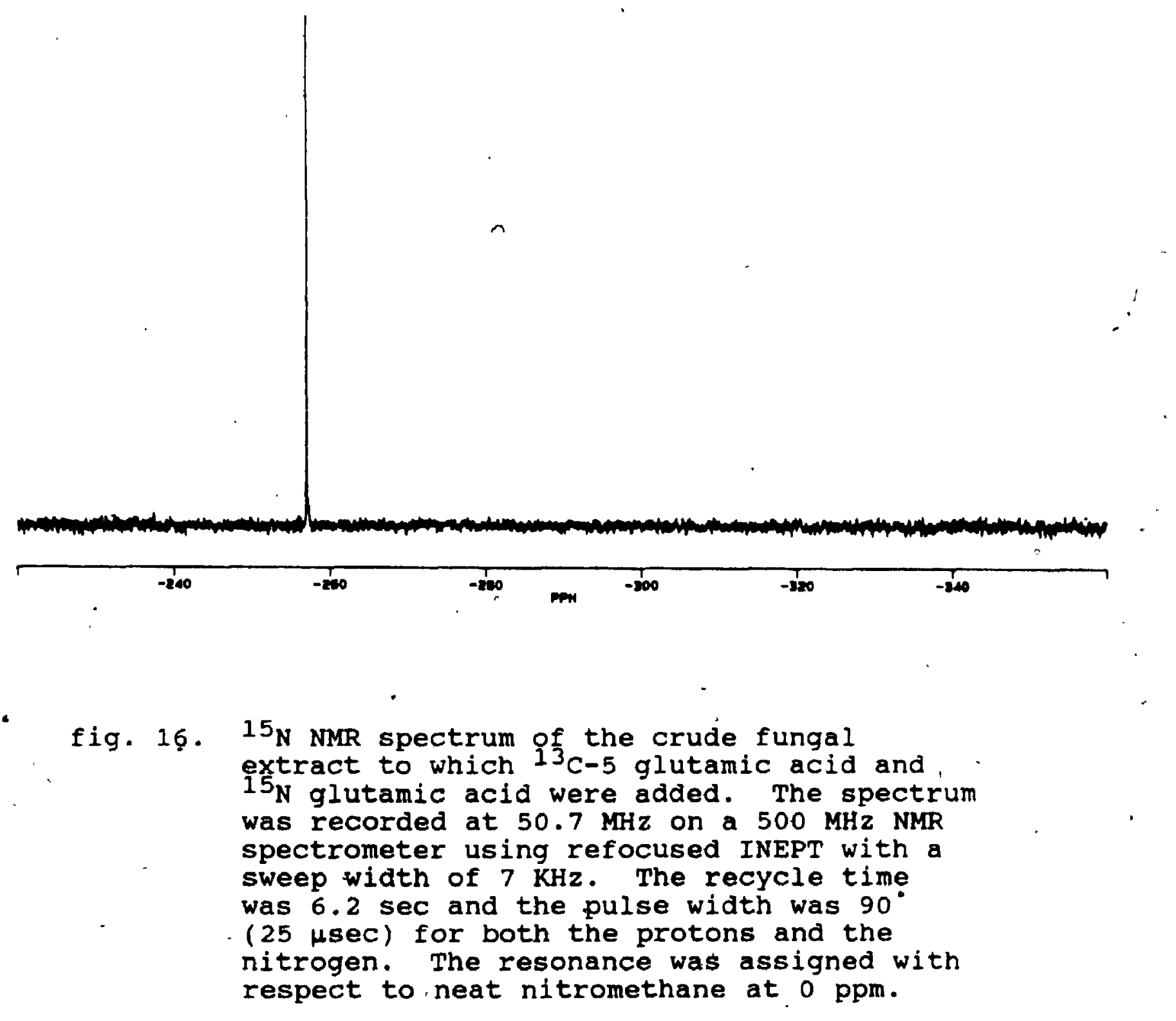

The above observations lead us to suggest that glutamic acid is a metabolic precursor of butenolide. A possible biosynthetic route is outlined in fig. 17 . 
Whis route involves reactions common to the metabolism of cells. The first step in the biosynthesis of butenolide from glutamic acid would involve a desaturation at carbon 3 of glutamic acid. The specific desaturation to the cis double bond at this position would make the cylization via decgrboxylation more favorable by bringing the two carboxylic acid termini closer together and therefore in a bettier orientation for the cyclization. After the cyclization has occurred, N-acetylation is all that would be required to produce butenolide. The ${ }^{13} \mathrm{C}$ enrichment pattern in butenolide supports this biosynthetic scheme as indicated in figure 17.

4-acetamido-2-butenoic acid, reported by Vesonder et al. (19). may be an intermediate in the biosynthesis of butenolide occurring as a transitional compound between the decarboxylation and the cyclization of the desaturated glutamic acid. The N-acetylation could result prior to the cyclization to give the observed intermediate. The observation of 4-acetamido-2butenoic acid, however, is a good indication that the desaturation occurs early in the biosynthesis of butenolide. 


\section{BIOSYNTHESIS OF BUTENOLIDE.}

D
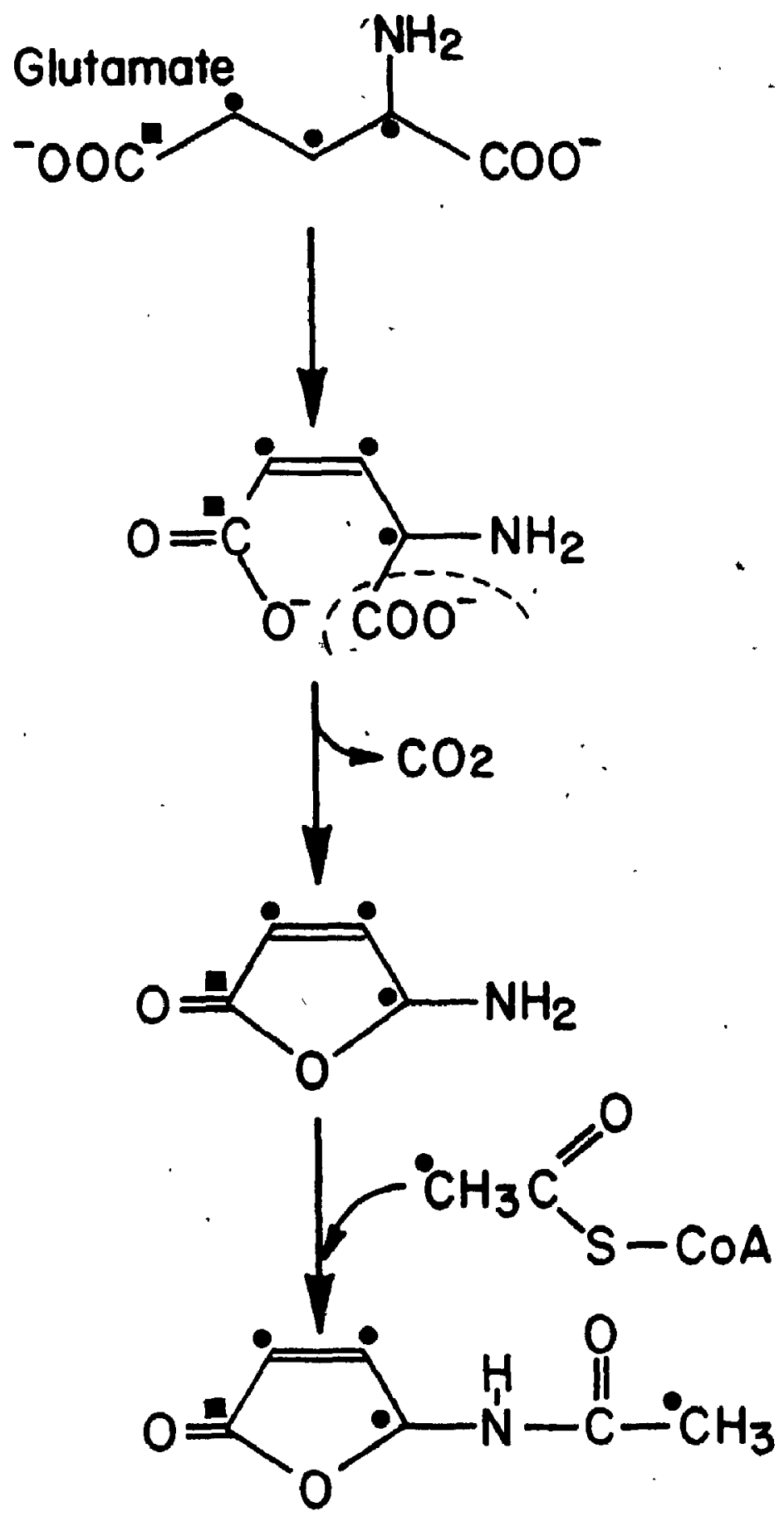

$\therefore \quad$ Butenolide

fig. 17 Proposed biosynthetic route to butenolide from glutamic acid showing the incorporation of $13 \mathrm{C}-5$ glutamic acid $($ ) and

${ }^{13} \mathrm{C}-2$ acetate $(\bullet$,$) and the resultant$ enrichment pattern in butenolide. 


\section{2-5 INVESTIGATION OF THE DEGRADATION OF BUTENOLIDE}

It was determined in our laboratory that the concentration of butenolide (1) in a $10 \mathrm{~L}$ stirred jar fermentor culture of E, culmerum HLX 1503 reached a maximum of ca. $300 \mathrm{mg} \mathrm{L}^{-1}$ at about $36 \mathrm{~h}$, declined to $75 \mathrm{mg} \mathrm{L}^{-1}$ by $78 \mathrm{~h}$ and was not detected by $168 \mathrm{~h}(20)$. The work performed thus $f a r$ in the shake flask does not exhibit this decline in the butenolide (1) concentration which continues to increase up to $72 \mathrm{~h}$. The cultures grown in the stirred jar fermentor grow fastér and survive longer than the shake flask cultures due to the greater control permitted over some of the growth. conditions. For example, the $\mathrm{pH}$ is maintained at 4 in the fermentor and therefore allows the culture to grow for a longer period of time producing more toxin than is possible in shake flask experiments: Decline of Butenolide concentration in shake Flask
Cultures

An experiment was therefore designed to see if the decline in the butenolide concentration encountered in stirred jar fermentations of F. culmorum HLX 1503 could be observed" under shake flask "conditions. In this 
experiment, 40 flasks were inoculated and incubated for $169 \mathrm{~h}$. Triplicate $50 \mathrm{~mL}$ cultures were harvested and analyzed separately at $12 \mathrm{~h}$ intervals starting at $24 \mathrm{~h}$ as described in section $3-1$ and 3-1a.

Both butenolide (1) and 3-ADON (3) were quantitated and it was noted that there was no definitive drop in the butenolide concentration after the initial rise (fig. 18). However, the concentration of butenolide did level off and remain relatively constant after about $84 \mathrm{~h}$. The lack of any drop in the concentration is likely due to the very poor growth conditions and near death of the cells after $96 \mathrm{~h}$ as indicated by the low $\mathrm{pH}$ (fig- 18). The large standard deviation increased with time. The deviation is experimentally insignificant at the beginning of the experiment and increases with time. The culture conditions within each shake flask cannot be controlled so they develop independently and therefore slightly differently. This is the major cause of the large deviation in the toxin concentration as time increases. 


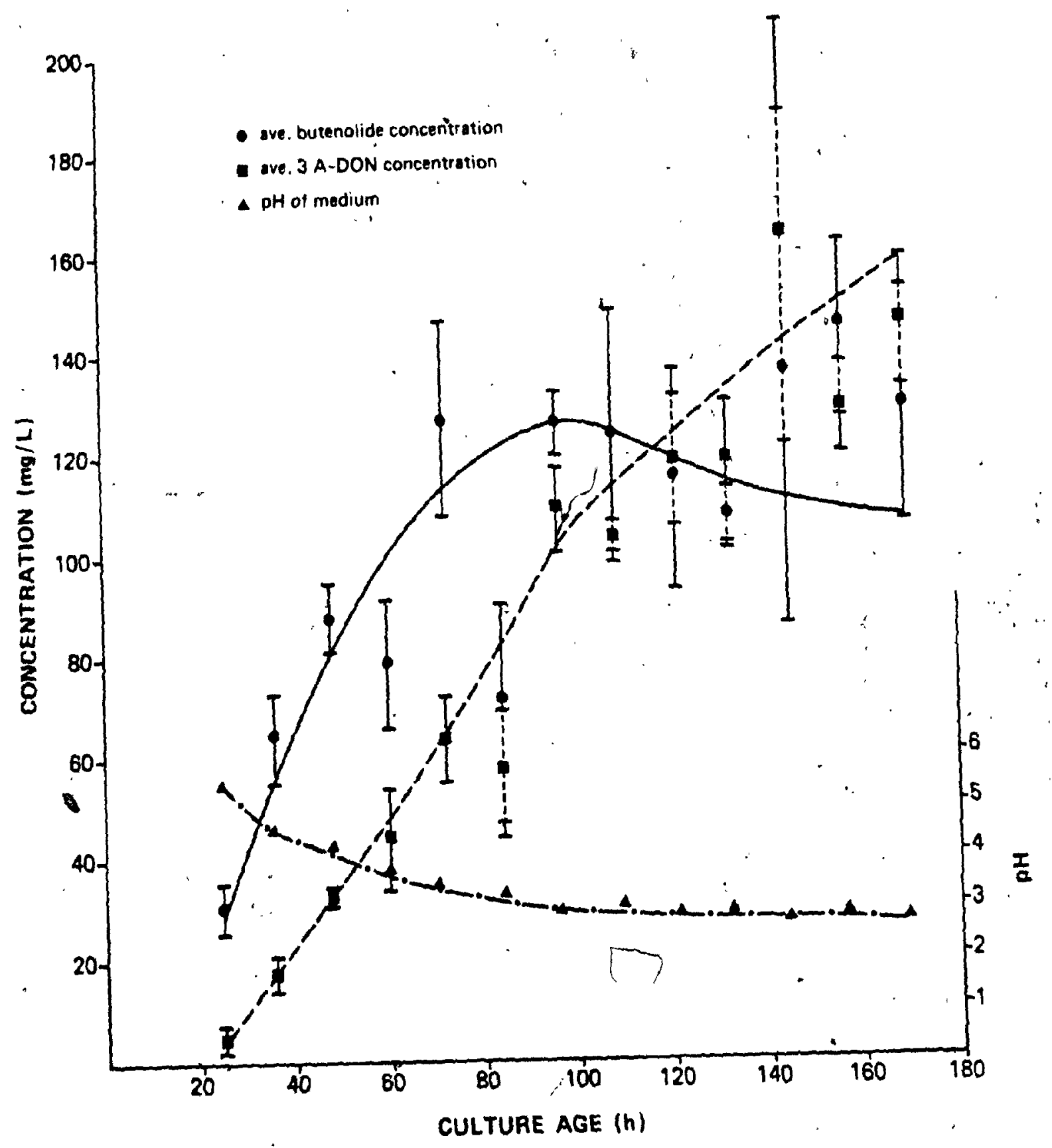

fig. 18 Profile of butenolide and 3-ADON concentration in $50 \mathrm{~mL}$ shake flask cultures of $E$. culmorum MIX 1503 as a function of culture age.

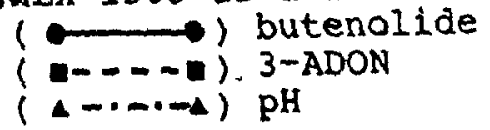




\section{2-6 FERMENTTOR EXPERIMENTS}

The 10 L stirred jar fermentor is a far superior method of monitoring the metabolism of a growing fungus. Using the fermentor, one can monitor and control the pH of the medium, the oxygenation and the temperature of the culture as well as record and store data at discrete time intervals for the duration of the experiment. However, since the operation of the fermentor is very expensive and requires large amounts of enriched compounds to obtain the desired concentration to run experiments, the parameters are first optimised.with shake flask cultures. This kept the number of times that the fermentor was required to a minimum.

Translating the information obtained from the shake flask experiments into appliaation for the fermentor requires'intimate knowledge of the growth of the fungal culture in both systems. In general, cultures. in the fermentor tend to develop faster than the shake flask cultures primarily because of the control over the pH afforded by the fermentation apparatus. As, the pH drops with the shake flask cultures the fungal metabolism slows down. The cells must maintain. a pH, gradient of up to $4 \mathrm{pH}$ units (from about $\partial .5$ inside the cell to 2.5 in the medium of shake flask cultures) 
across the cel membrane. This requires energy and puts. "an. added on the cell metabolism. BY extenaliy maintaining the $\mathrm{pH}$ of the medium at 4 the cells are able to survive ronger and therefore toxin production çan be continued for a longer period of tige at an increased level.

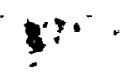

Metabolism study - monitoring through the use of $13 \mathrm{C}-2$ acetate.

The best precufsor for the metabolism studies of $F$. culmorum HLX 1503. for this project, is $13 \mathrm{C}-2$ acotate because the incorporation of this precursor into, the. adjacent, carbon atoms, $c-2, c-3, a$ and $c-4$, in butenolide (1) and into the trichothecenes permits simultaneous investigation of the TCA "cyche and the mevalonic acid pathway. The data obtained from ${ }^{13} \mathrm{C}$ NMR spectróscopy of the crude fungal extracts allows the calculations of a) the extent of enrichment of both butefiblide and 3-ADON, b) the ratio of multiple to single enrichment in butenolide and c) the per cent of triple enrichment (ie. all three adjacent carbon atoms enriched) in qutenolide. These calculations permit the study of the efficiency of the TCA cycle, the activity of the glyoxylic acid shunt and analysis of mevalonic acid cycle activity at any one time by using the enicichment 
patterns in the products of these pathways as a guide. Figure $19^{\circ}$ shows the ${ }^{13} \mathrm{C}$ NMR coupling pattern of the resonances of the three adjacent carbon atoms of butenolide used to determine the level of enrichment of this TCA cycle product.
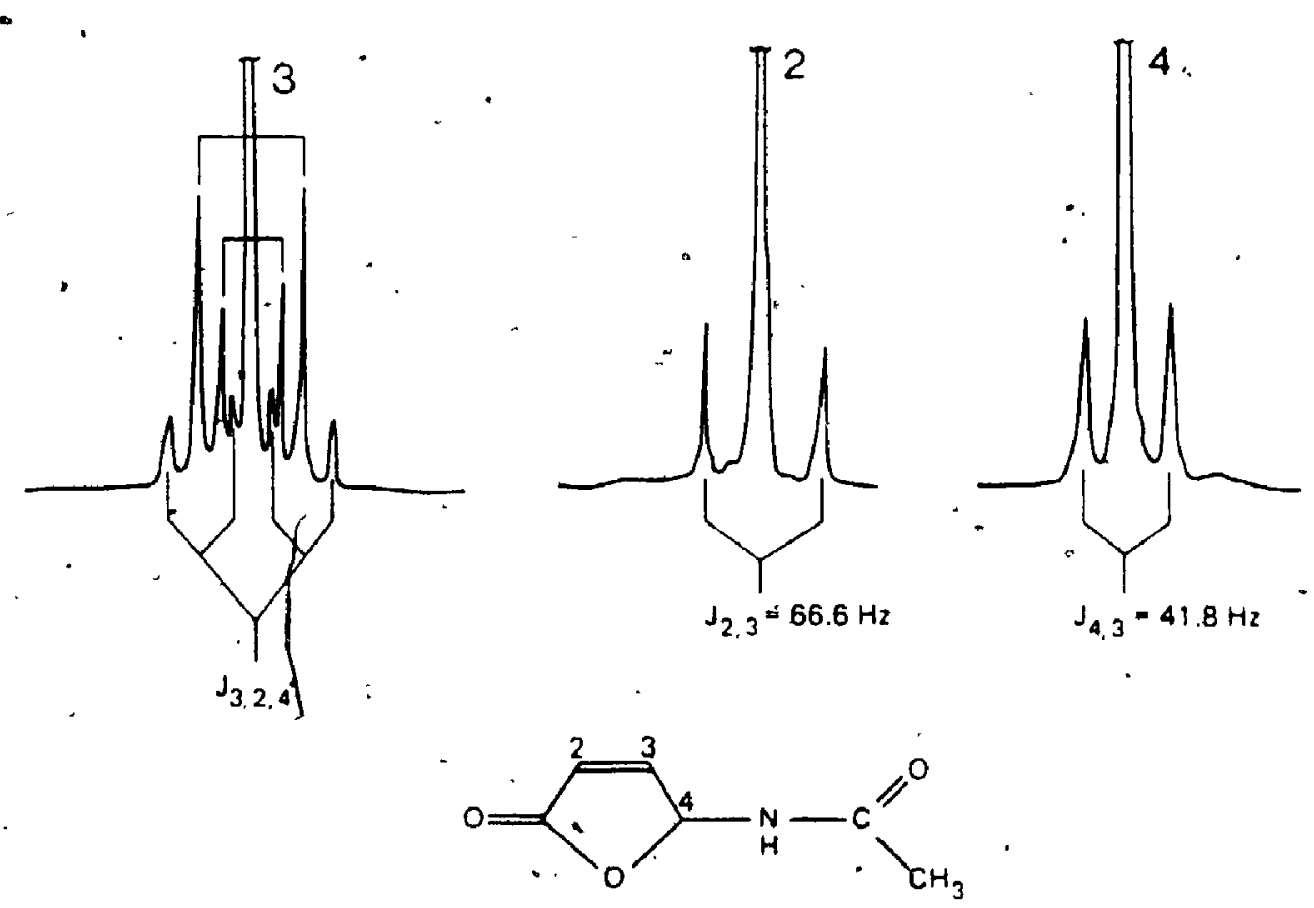

fig: 19. Peaks and coupling constants for. the three adjacent carbon atoms, $\mathrm{C}-2, \mathrm{C}-3$ and $\mathrm{C}-4$, of butenolide used for the calculations for level of enrichment.

The activity of the TCA cycle and the glyoxylic acid shunt can be studied using the enrichment pattern of the adjacent enriched carbons $\mathrm{C}-2, \mathrm{C}-3$ and $\mathrm{c}-4$ of butenolide whereas the 3-ADON (3) enrichment pattern is 
a good indicator of mevalonic acid cycle activity. The carbons at positions 2 and 3 of $3-A D O N$ (3) can be used to determine the extent of enrichmegt in this compound (fig. 20).

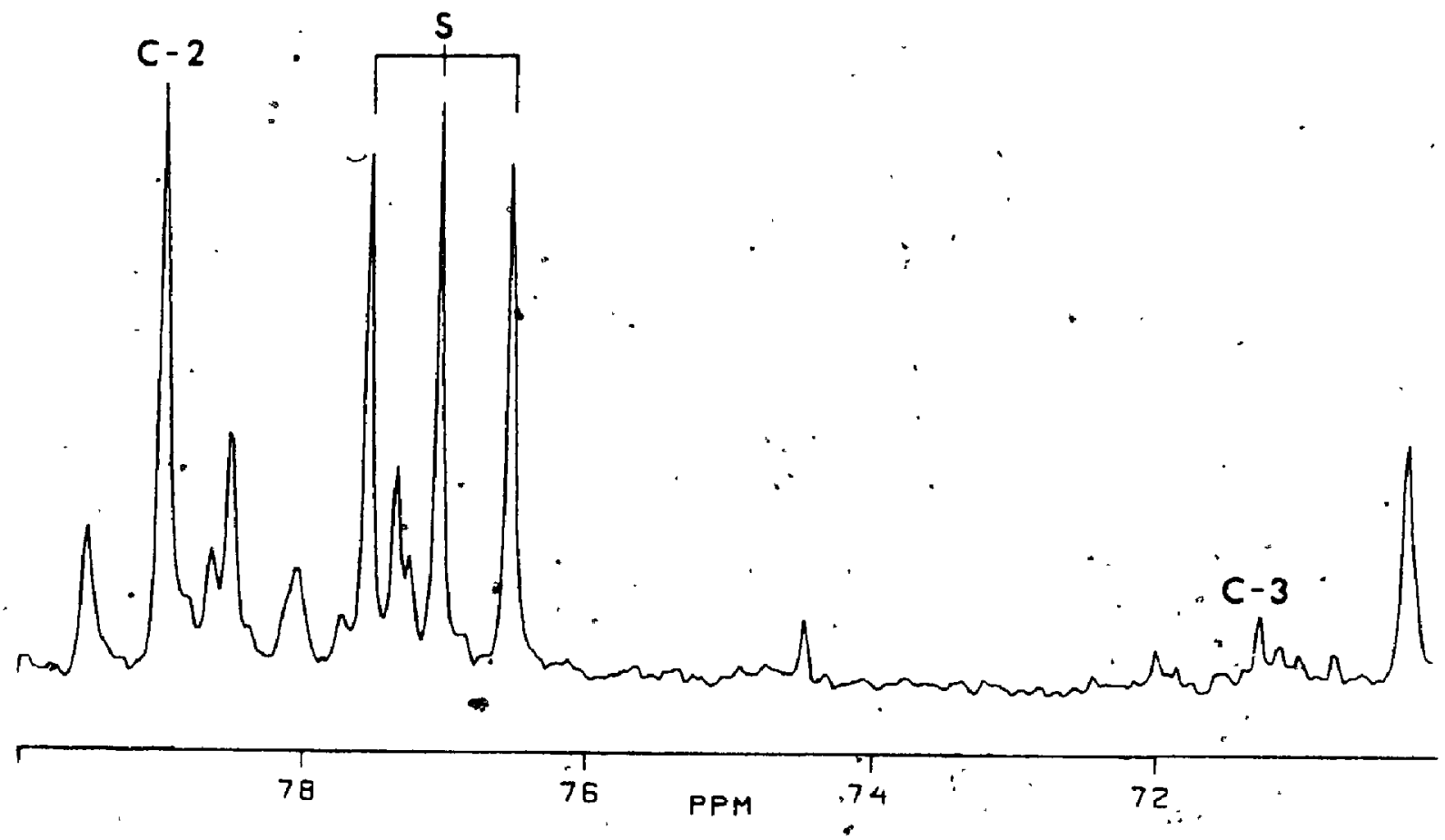

fig. 20 Expansion of the $42 \mathrm{~h}^{13} \mathrm{C}$. NMR spectrum (fig. 23) showing the resonances used to determine the level of enrichment in 3-ADON. $S$ sotvent. $\mathrm{C}-2$ and $\mathrm{C}-3$ refer to the carbon atoms of these positions in 3-ADON (3).

Carbon 2 becomes enriched from ${ }^{13} \mathrm{C}-2$ acetate whereas carbon 3 will remain unenriched. These calculations are only executed using the ${ }^{13} \mathrm{C}$ NMR data of the crude 
exträcts from experiments performed with the stirred jar fermentor.

The purpose of running experiments in the stirred-jar fermentor, was to monitor the metabolism. of the fungus at discrete time intervals by ${ }^{13} \mathrm{C}$ NMR. This can be achieved by removing samples from the fermertor; extracting them and taking the NMR spectrum of the crude fungal extract. This essentially gives a picture of the metabolism at that time through the metabolic products, their enrichment patterns and the ratio of butenolide (1) -to trichothecenes. The metabolism of praducts of the TCA, cycle, such as butenolide, and of the mevalonic acid cycle, such as, the trichothecenes, can be followed and analyzed.

Experiments to study the ${ }^{13} \mathrm{C}-2$ 'Acetate Incorporation into the Metabolic products of 'E.culmorum HLX 1503: Experimènt 1 (FR-1)

The ${ }^{13} \mathrm{C}=2$ acetate was added to the fermentor over $a$ $24 \mathrm{~h}$ period in 5 equal aliquots starting at $12 \mathrm{~h}$. The enriched acetate was added at $12 \mathrm{~h}$ rather than at $24 \mathrm{~h}$ which was desirable for. the shake flask experiments, because the fungal fementation in the fermentor is initially quickes than the shake flask fermentations. The final concentration of ${ }^{13} \mathrm{C}-2$ acetate added was 0.8 
$\mathrm{mg} / \mathrm{mL}$ (a total of $8 \mathrm{gm}$ were added to the $10 \mathrm{~L}$ in the fermentor). Samples were taken every $6 \mathrm{~h}$ as described in section $3-2$. The ${ }^{13} \mathrm{C}$ NMR spectra were acquired for each crude fungal extract.

The concentrations of butenolide (1) and 3-ADON (3) for this fermentation are shown in figure 21 At $48 \mathrm{~h}$, when the butenolide concentration had peaked and started to decrease the 3-ADON (3) production started and continued to rise. This observation is consistent - with the information from figure 2 (section 1) and with previous data reported by our laboratory (20).

The ${ }^{13} \mathrm{C}$ enrichment at each carbon atom in butenolide for this experiment is given in Table 7 .

Table 7. Total ${ }^{13} \mathrm{C}$ enrichment factor in the carbon atoms of butenolide from the first fermentation experiment (FR-1).

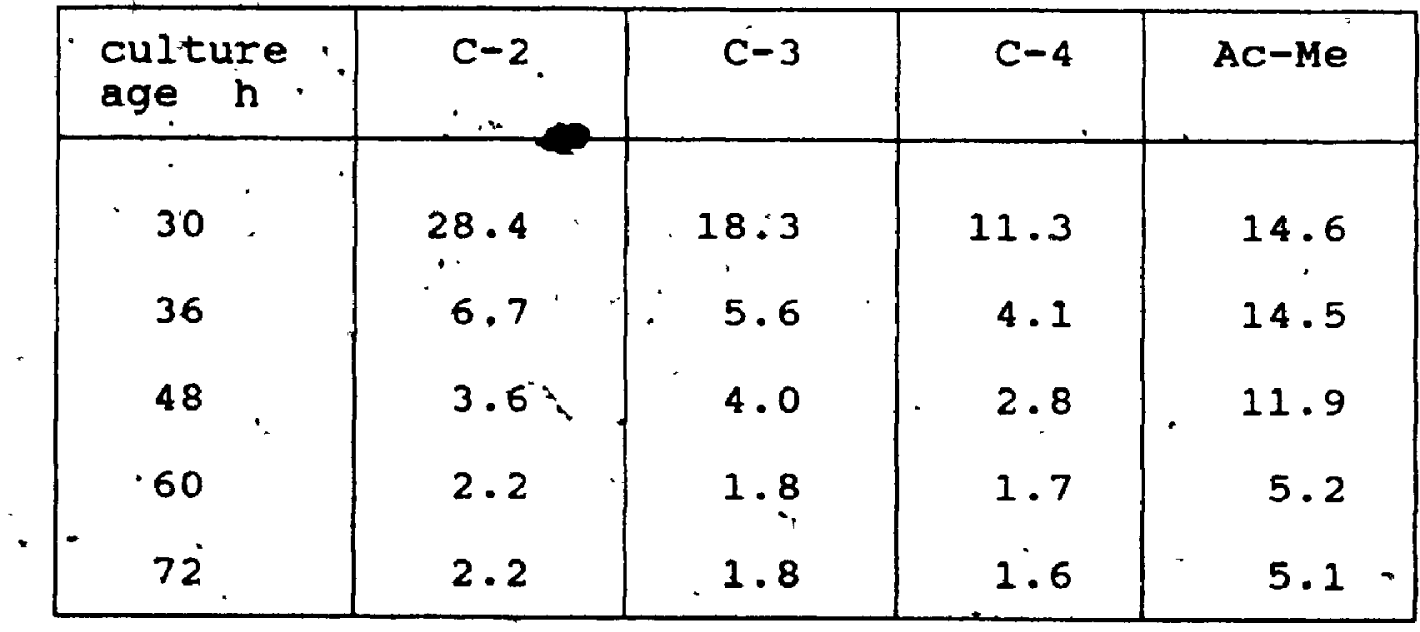

2 For a description of the calculations used to obtain the data for tables 7,8 and 9 , see appendix 1 . 


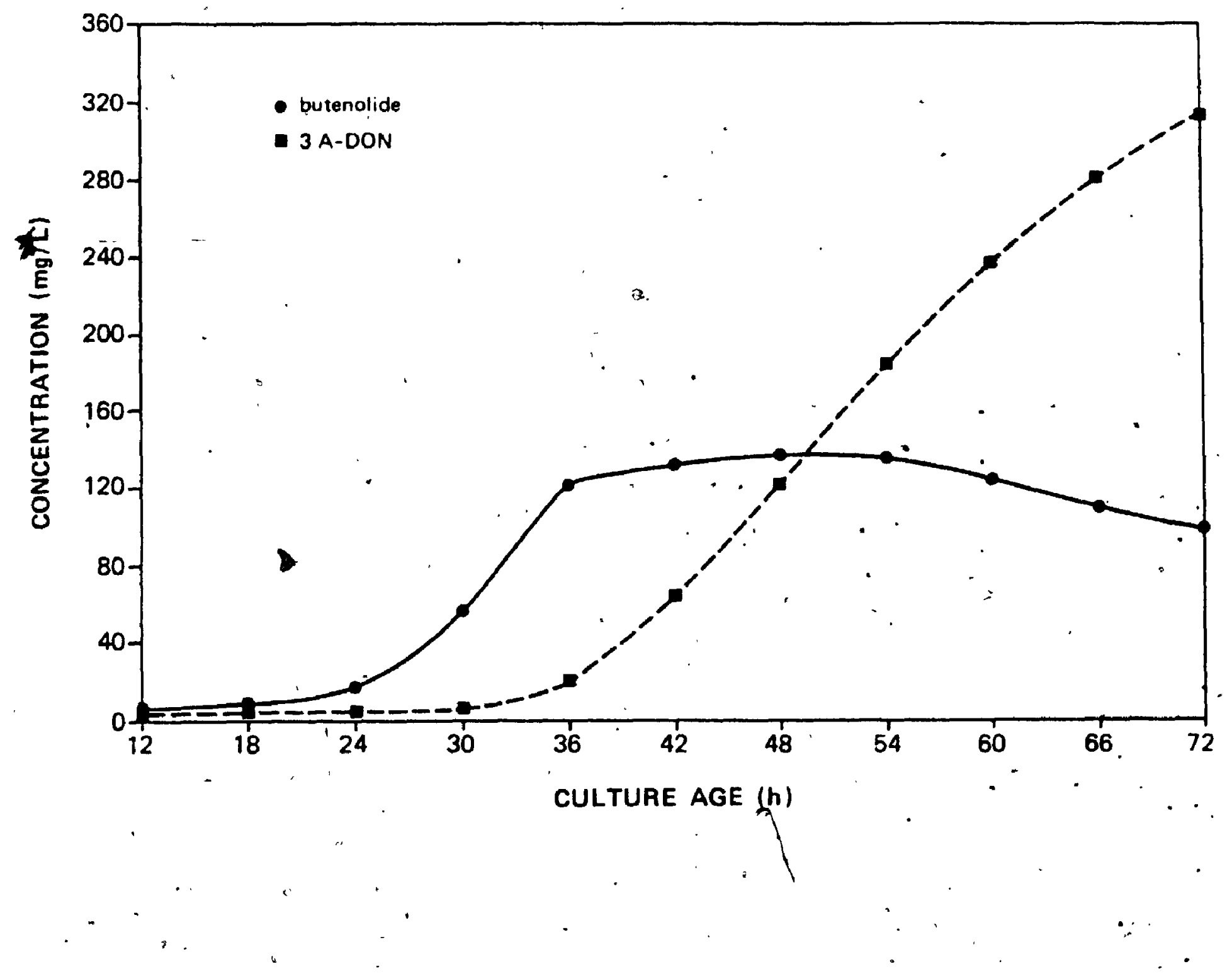

fig. 21 The yield of butenolide and 3-ADON as a function of culture age from FR-1. butenolide $(\bullet-)^{\prime}$ 3-ADON $(--\rightarrow)$.<smiles></smiles> 
The level of enrichment of butenolide was highest at $30 \mathrm{~h}$ and then dropped to a constant level at $60 \mathrm{~h}$ (table 7). At $36 \mathrm{~h}$, even though the enriched acetate. was still, being added, the incorporation into butenolide decreased and some incorporation into 3-ADON was observed by ${ }^{13} \mathrm{C}$ NMR spectroscopy (fig. 22). This is indicative of the onset of trichothecene production between $30 \mathrm{~h}$ and $36 \mathrm{~h}$ and this pathway is now competing for the enriched acetate. Between $36 \mathrm{~h}$ and $60 \mathrm{~h}$, the level of enrichment decreases slowly due to the dilution, of the enriched acetate with unlabelled species. The level of enrichment in bulenolide at $60 \mathrm{~h}$ and $72 \mathrm{~h}$, (table 7) was constant indicating that the production of butenolide had stopped.

At $30 \mathrm{~h}$, the enrichment at $\mathrm{c}-2$ is significantly higher than the other positions indicating that glutamate is being produced and used very efficiently and the enriched methyl group only remains in the TCA cycle for one pass around the cycle (fig. 7 , section 1). After $30 \mathrm{~h}$, this difference between enrichment at $c-2, c-3$ and $c-4$ of butenolide diminishes which indicates that the glitamate is becoming multiply enriched. Therefore, the enriched acetate molecule must be remaining in the TCA cycle for more than one pass indicating that glutamate is not being used as efficiently. 
fig. $22{ }^{13} \mathrm{C}$ NMR spectra of the crude fungal extracts of the first fermentation experiment of E. sulmorum HLX 1503 (FR-1). These spectra were acquired with the same parameters as the. natural abundance spectrum. Since the spectra are plotted relative to one another, the peak intensities can Be compared from spectrum to spectrum. The spectra were also plotted so the smaller peaks could be observed causing the larger peaks to be truncated as indicated with the $\sim$. A 3-ADON; B butenolide. The numbers refer to the carbon atom of the molecule indicated which is observed. Note the increase in the resonances of 3-ADON and the increase and decrease in the resonances of butenolidé over time. 


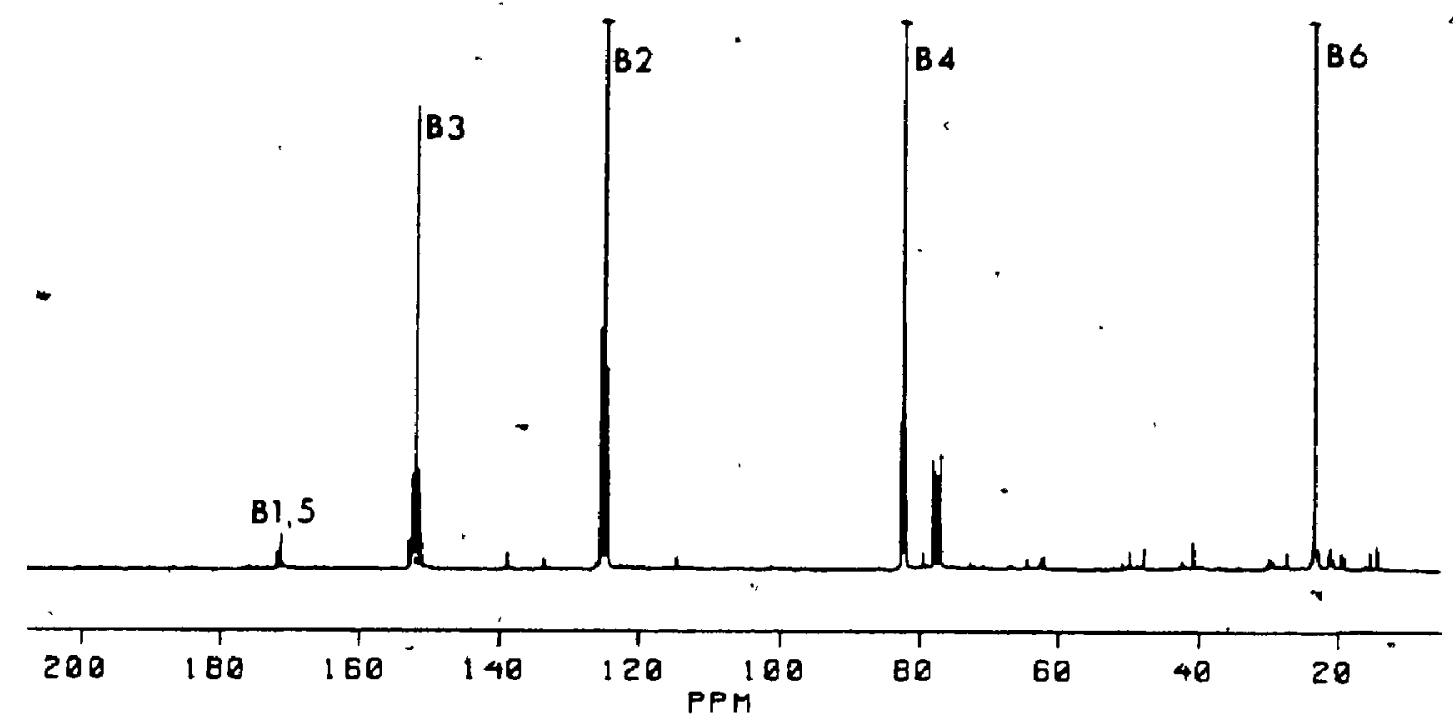

B) 36 HOURS

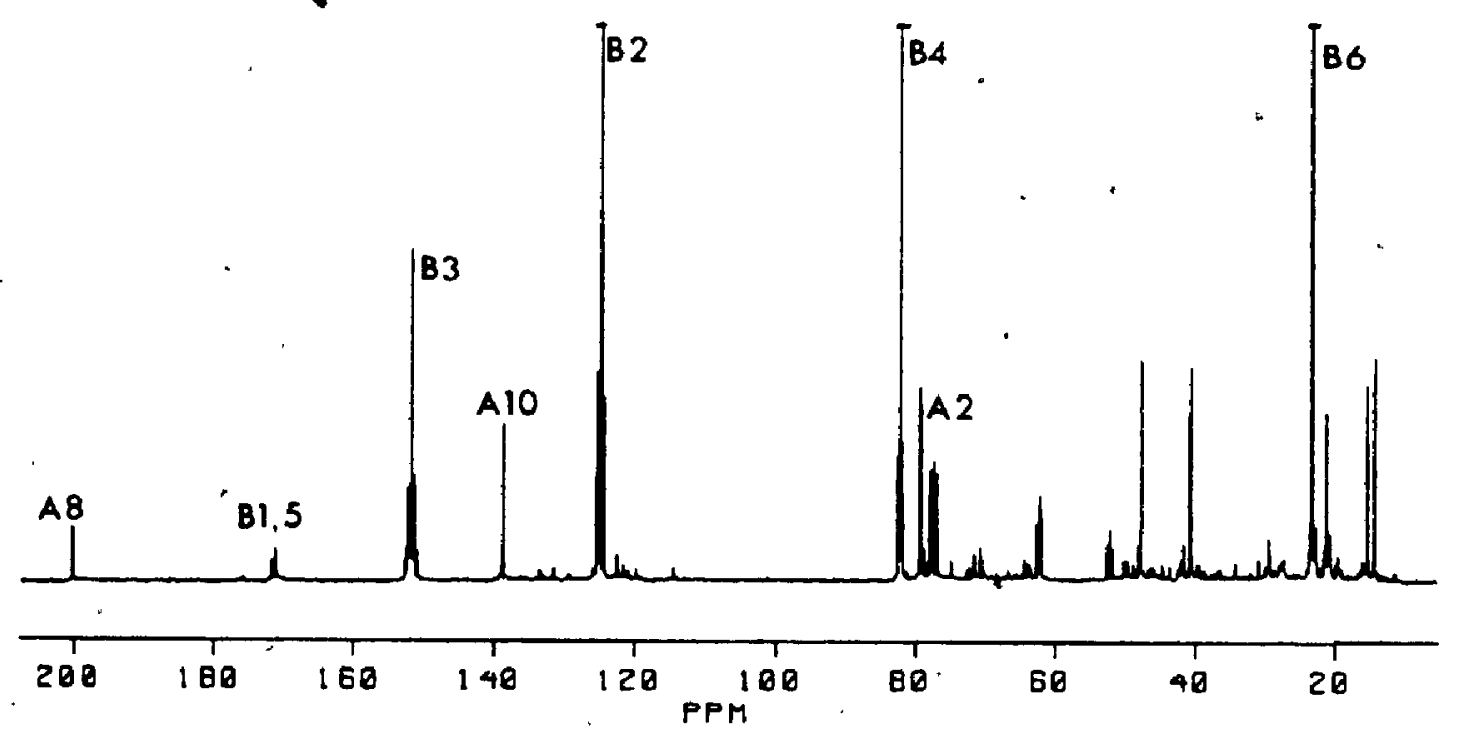

C1 - 92 HOURS

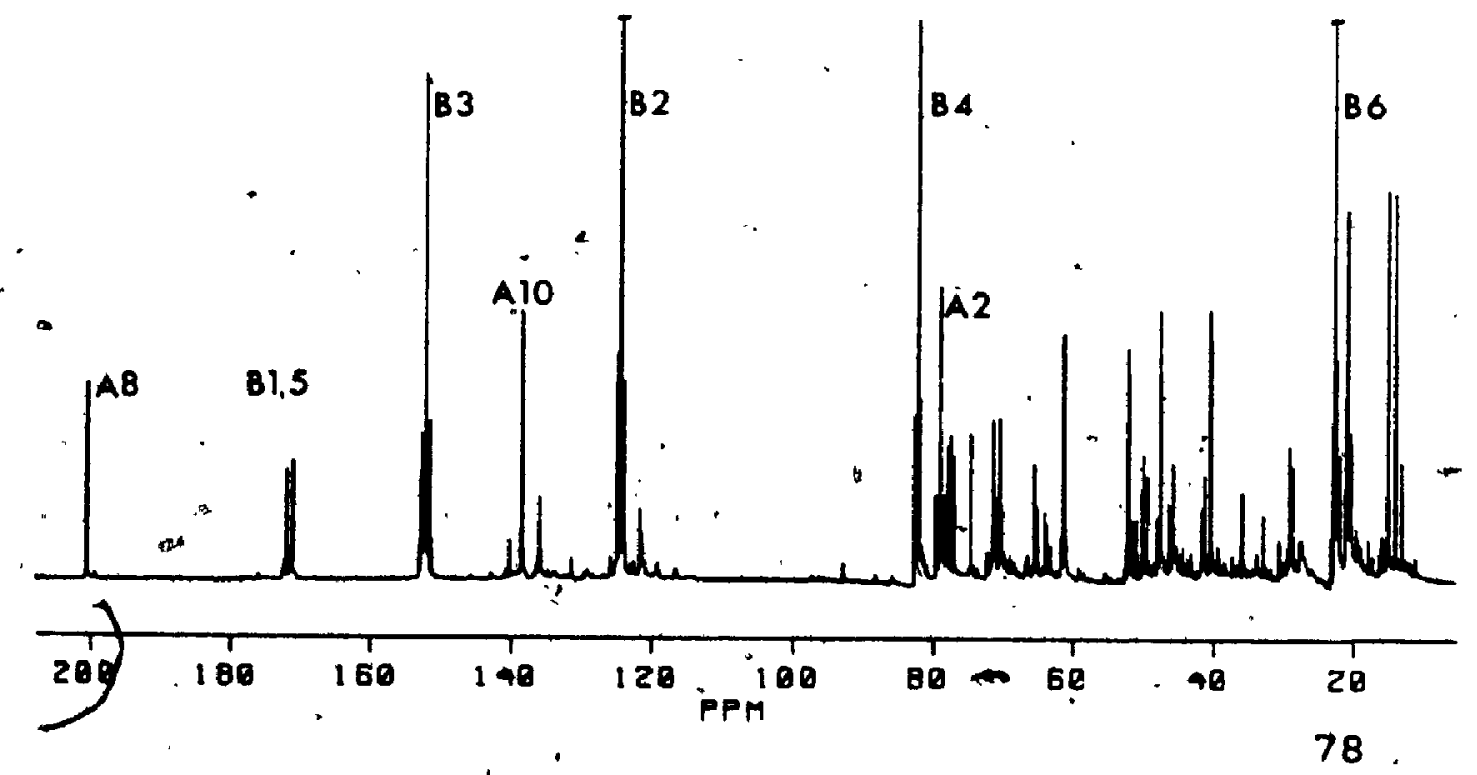




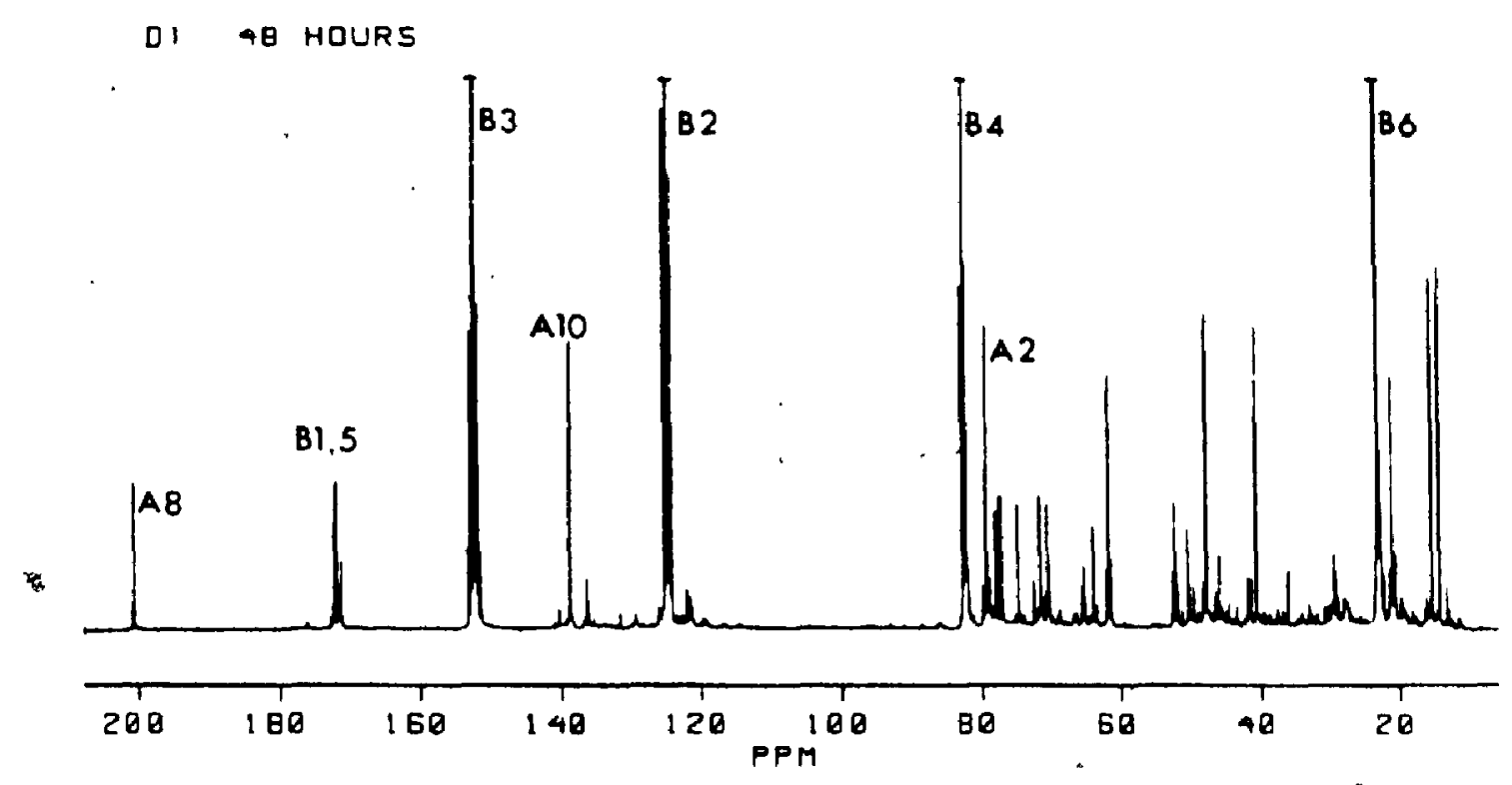

E) 68 HOURS

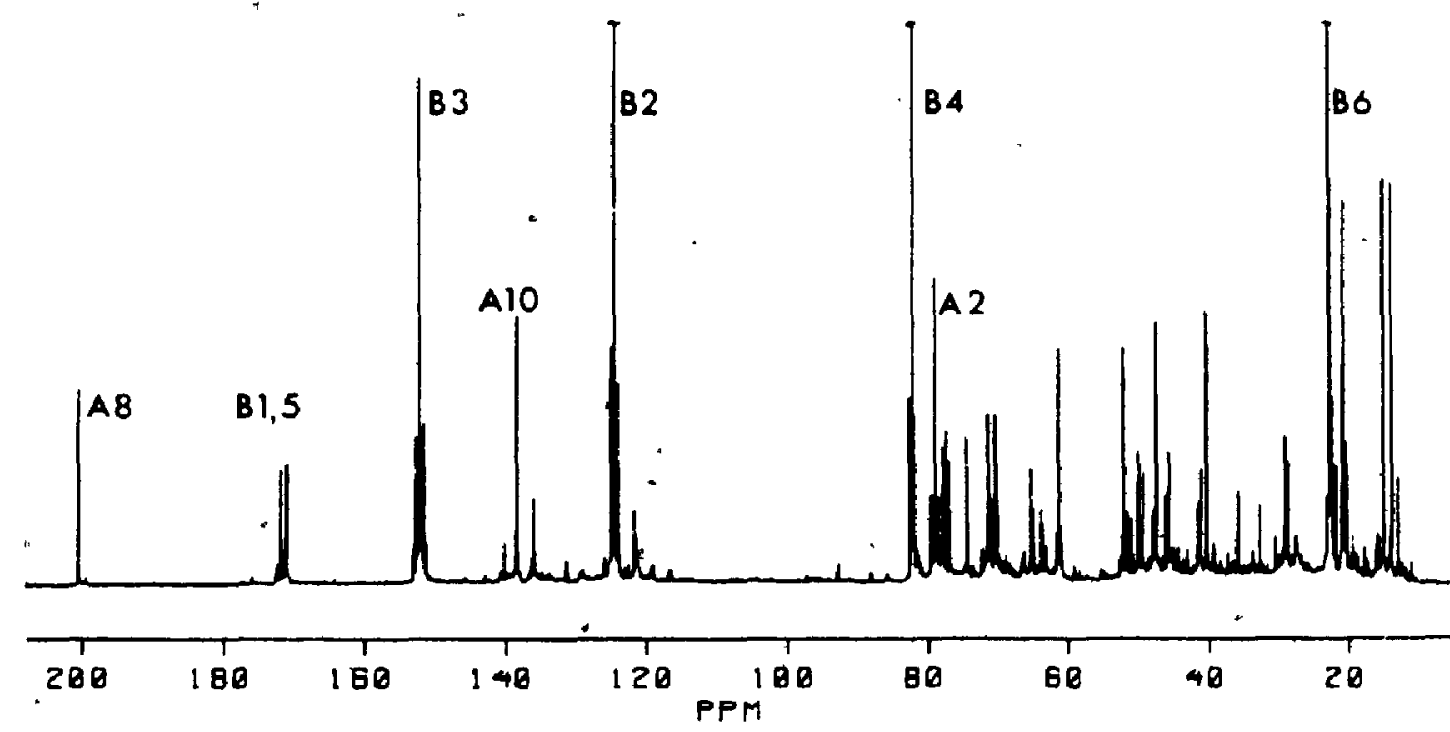

FI' 72 HOURS.

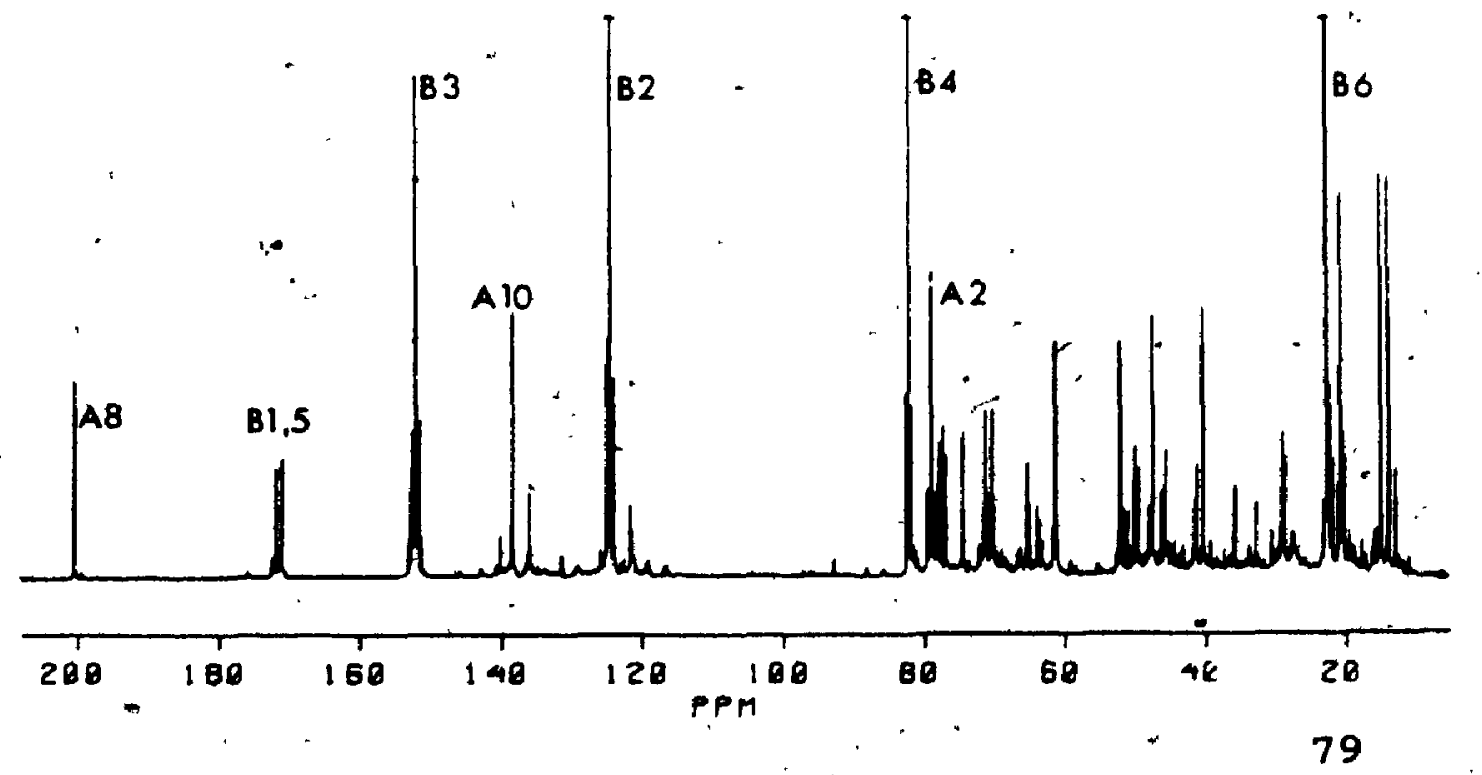


The enrichment at the Ac-Me decreases (table 7) with time due to the dilution of the ${ }^{13} \mathrm{C}$-acetate pool within the cell with unenriched species. It is the same order of magnitude at $30 \mathrm{~h}$ but is higher at $36 \mathrm{~h}$ and $48 \mathrm{~h}$ relative to the other positions. This shows that there were more enriched species available for free acetylation at this time than at $30 \mathrm{~h}$. The reason for this is likeIy the decreased activity of the TCA cycle and increased competition for the acetate by the mevalonic acfid cycle.

The activity of the glyoxylate shunt can be determined from the enrichment pattern of butenolide as well. If the glyoxylate shunt is active, then C-4 will be the most enriched (fig. 7, section 1) followed by c-3 and c-2. Carbon 4 does not exhibit the highest level of enrichment (tabie 7) which indicates that the glyoxylate shunt is not very active. 
Table $\overline{8}$. Ratio of multiple to single enrichment in butenolide enriched with ${ }^{13} \mathrm{C}-2$ acetate. Data is from the FR-1.

\begin{tabular}{|c|c|c|c|}
\hline $\begin{array}{l}\text { culture } \\
\text { age } h\end{array}$ & $c-2$ & $\Rightarrow \quad c-3$ & $c-4$ \\
\hline $30^{\circ}$ & .30 & $.70(.40)$ & .50 \\
\hline 36 & .44 & $1.48(.37)$ & .45 \\
\hline 48 & .52 & $2.42(.31)$ & .52 \\
\hline 60 & .20 & $1.64(.30)$ & .31 \\
\hline 72 & .20 & $1.61(.30)$ & .31 \\
\hline
\end{tabular}

The ratio of multiply enriched species increases slightly up to $48 \mathrm{~h}$ then drops and remains constant after this time (table 8 ). The higher the level of multiple enrichment, the more passes the enriched acetate makes around the TCA cycle. Therefore, the glutamate is not being used as quickly as it is at times when the multiple enrichment is lower. This indicates that the glutamic acid is not being used for butenolide synthesis as quickly as the TCA cycle can produce $\alpha$-ketoglutaric acid (an immediate precursor from the TCA cycle of glutamic acid) which must then remain in the cycle and become further enriched until there is need for it. The utilization of glutamic acid for either protein or butenolide synthesis provides the outlet from the TCA cycle for a-ketoglutaric acid". ' 
The numbers in brackets under $\mathrm{C}-3$ are the ratio of double, to triple enrichment. This ratio can be calculated since the coupling at $\mathrm{C}-3$ is completely resolved (see appendix 1 for details of the calculations). This number remains fairly constant which shows that the label survives more passes around the TCA cycle before glutamate is used for butenolide (1) or protein synthesis demonstrating the reduced demand for glutamate.

Experiment $2(\mathrm{FR}-3)$.

A similar experiment was run in the fermentor involving the addition of ${ }^{13} \mathrm{C}-2$ acetate over a $24 \mathrm{~h}$ periog starting at. $12 \mathrm{~h}$. Samples were taken every $6 \mathrm{~h}$ except that between $28 \mathrm{~h}$ and $42 \mathrm{~h}$ samples were taken at $2 \mathrm{~h}$ intervals. The purpose of the $2 \mathrm{~h}$ samples was to more closely monitor and analyze the time when butenolide production decreased and trichothecene production increased. The dynamics of enrichment in butenolide and 3-ADON could also, be more closely monitored in this way.

Some sample ${ }^{13} \mathrm{C}$ NMR spectra from this experiment are shown in figure 23. Comparison' with figure 22 shows that these two fermentation experiments were very similar with respect to toxin production and label incorporation. 
.

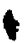

fig. $23{ }^{13} \mathrm{C}$ NMR' spectra of crude fungal extracts of samples from FR-3. A 3-ADON; B butenolide. The numbers refer to the positions of the carbon atoms in the molecules. The indicates resonances which were truncated when the spectra were platted. All of the spectra are again plotted with respect to each other so the intesities can be compared. The 1. indicates 3-ADON resonances which were used to determine the level af enrichment in this molecule. The three spectra are shown as examples from this experiment.

\section{4} 1

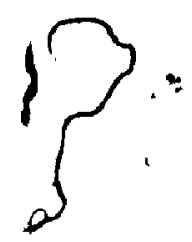




$$
2^{\text {oflde } 2}
$$




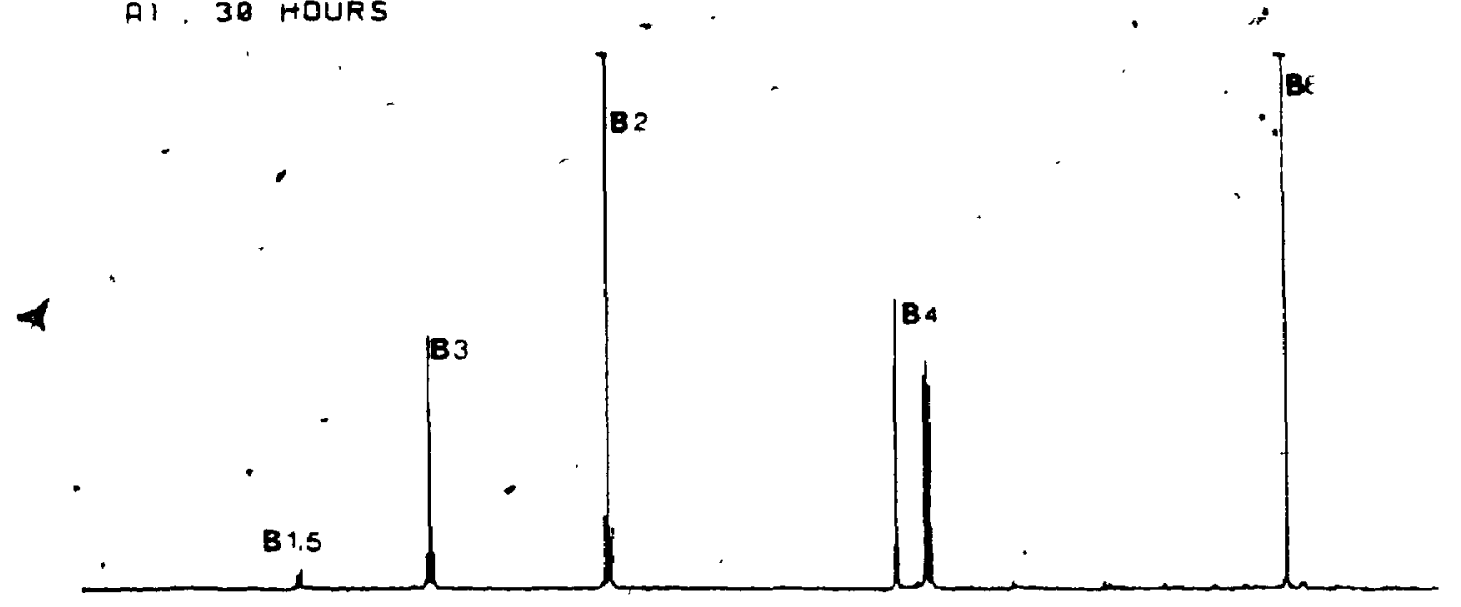

3

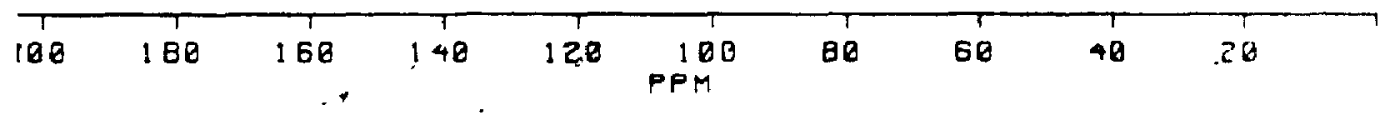

BI 42 HOUR5

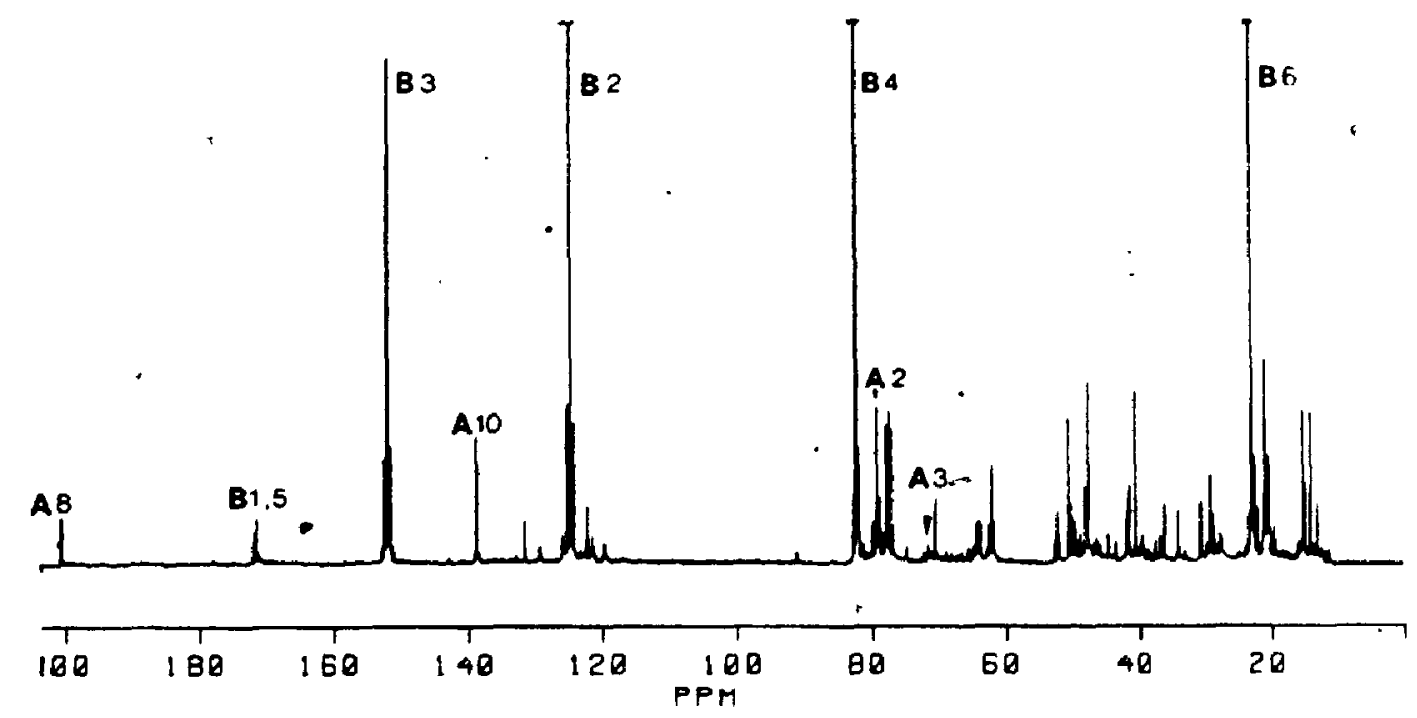

CI 54 HOURS

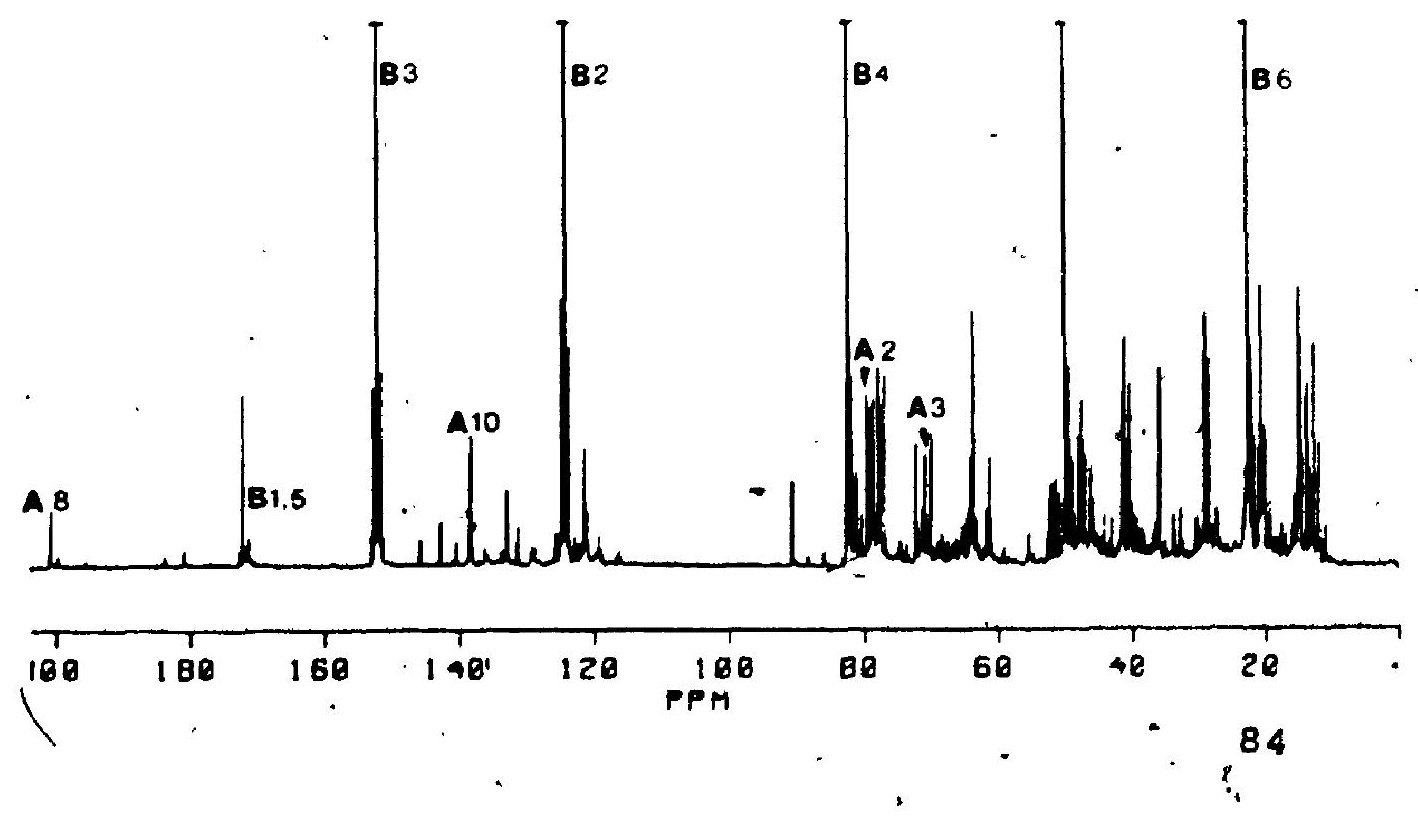


Table 9. ${ }^{13} \mathrm{C}$ enrichment of the adjacent carbion. atoms of butenolide as compared with the enrichment in,3-ADON. Data obtained from FR-3.

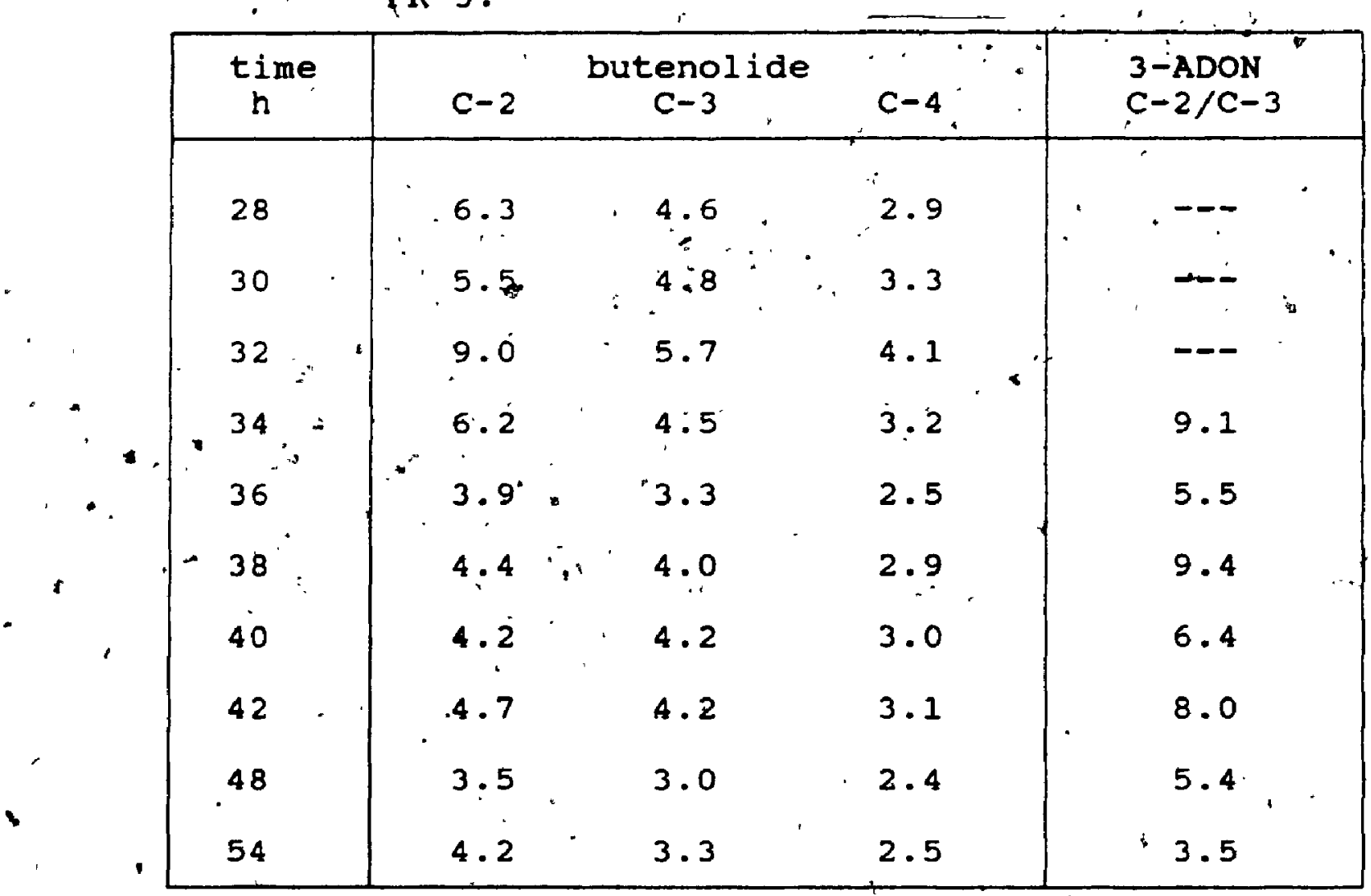

To understand the apparent variability in the extent of ${ }^{13} \mathrm{C}$ enrichment of table 9; it is necessary to know when the enriched acetate was added. The addition times "were $12 \mathrm{~h}, 18 \mathrm{~h}, 24 \mathrm{~h}, 30 \mathrm{~h}$, and $36 \mathrm{~h}$.' since. samples were extracted every two hours and the label was added to the fermentation in aliquots within these sampling times, the times "when label was added is of significance.

At $28 \mathrm{~h}$, the enrichment in $\mathrm{c}-2$ of butenolide was 6.3 whereas at $32 \mathrm{~h}$ it was 9.0 and at $34 \mathrm{~h}$ it was 6.2 . The 
level of enrichment at $28 \mathrm{~h}$ and $34 \mathrm{~h}$ is constant and both times are 4 hours after label was added. The high. level of enrichment at $3 \dot{2} \mathrm{~h}$ occurs because this time is only two hours after an enriched acetate aliquot had been added. Therefore, there was more label available just after addition than just before the subsequent addition. The acetate pool within the cells had a higher concentration of enriched acetate just after each addition and it was slowly diluted as unenriched acetate was produced maintaining the pool size.

"The same phenomenon" is observed for 3-ADON between

$.34 \mathrm{~h}$ and $40 \mathrm{~h}$. The level of enrichment of 3-ADON (3) at $28 \mathrm{~h}$ and $32 \mathrm{~h}$ is not available because the concentration of this molecule was too low at these times. At $38 \mathrm{~h}(2 \mathrm{~h}$ after the final addition), however, the level of enrichment in butenolide was low. The value for the enrichment after this time remains relatively constant indicating that the production of butenolide had stopped. The level of enrichment in 3-ADON remains high, though, indicating that the production of the trichothecenes is now much faster than the production of butenolide. The faster the metabolic pathway that is in operation produces products the more label there will be in these products because the two pathways compete for the same precursor and will act as sink for any acetate in the cell. If 
there $j=-13 \mathrm{c}$ enriched acetate in the system then this acetate will be used just as much as unenriched acetate from another source would be. The enrichment in 3-ADON fluctuates with the additions of the label as the enrichment in butenolide did when production was high. samples were always taken before the label was added when the two procedures were performed at the same time.

- The extent of triple enrichment was approximately the same as the first fermentation experiment described. The avtrage percent of triple enrichment for FR-1 was $13.3 \%$ and for FR-3 was 9.5\%. These numbers were relatively consistent over the time period. that the data were obtained.

Fermetation Studies of Butenolide Degradation.

The First Experiment (FR-2)<smiles>[13CH3]</smiles>

The study of the degradation of butenolide (1) was performed, twice in the fermentor. In the first experiment (FR-2) $133 \mathrm{mg}$ of ${ }^{13} \mathrm{C}$-butenolide vere added over a $24 \mathrm{~h}$ period starting at $36 \mathrm{~h}$ and $200 \mathrm{~mL}$ samples were taken at $24 \mathrm{~h}, 48 \mathrm{~h}, 60 \mathrm{~h}, 72 \mathrm{~h}, 84 \mathrm{~h}$, and $96 \mathrm{~h}$. The fermentor was harvested at $98 \mathrm{~h}$. The butenolide was added at $36 \mathrm{~h}$ because by this time the butenolide. 
concentration had peaked in the first fermentation experiment performed (fig.,21). The concentration of butenolide at the time of harvesting was stll fairly high at $45.8 \mathrm{mg} / \mathrm{L}$. The overall level of, butenolide production was not high in this fermentation experiment, peaking at $123.5 \mathrm{mg} / \mathrm{L}$ during theftime that the ${ }^{13} \mathrm{C}$-butenolide was being added.

The ressults from the NMR spectra of the crude fungal extracts. were inconclusive (same as 'fig. 24 , sample from FR-4). The original concentration of ${ }^{13}$ f-butenolide added to the fermentation was too low making any enriched products too dilute to be detected with NMR spectroscopy. 'This prevented any conclusion. as to the catabolism of butenolide ( 1 ).

.

'Precipitation of the Protein from the Mycelium from FR-2 .

Since no degradation products could be discerned from the ${ }^{13} \mathrm{C}$. NMR data of the crude fungal extracts from the first fermentor degradation experiment, the possibility that the enriched butenolide was broken down and reused either as a nitrogen source. or as an amino acid and recycled into protein synthesis was investigated. Thes was accomplished by precipitating the proteins from the mycelium of FR-4 and analyzing." 
the precipitate by ${ }^{13} \mathrm{C}$ NMR spectroscopy for any enrichment. The procedure for the precipitation of the protein from the mycelium of FR-2 is described in Section $3-3$ of this report. ${ }^{13} C-M M R$ analysis of the precipitated protein indicated that there were no enriched amino acids or other possible degradation products in this extract (fig. 24 ). The only products observed were lipids that were associated with lipoproteins. This may have been due to the Inadequate concentration of ${ }^{13} \mathrm{C}$-butenolide $j . n$ the fermentation as mentioned above.

$\therefore$

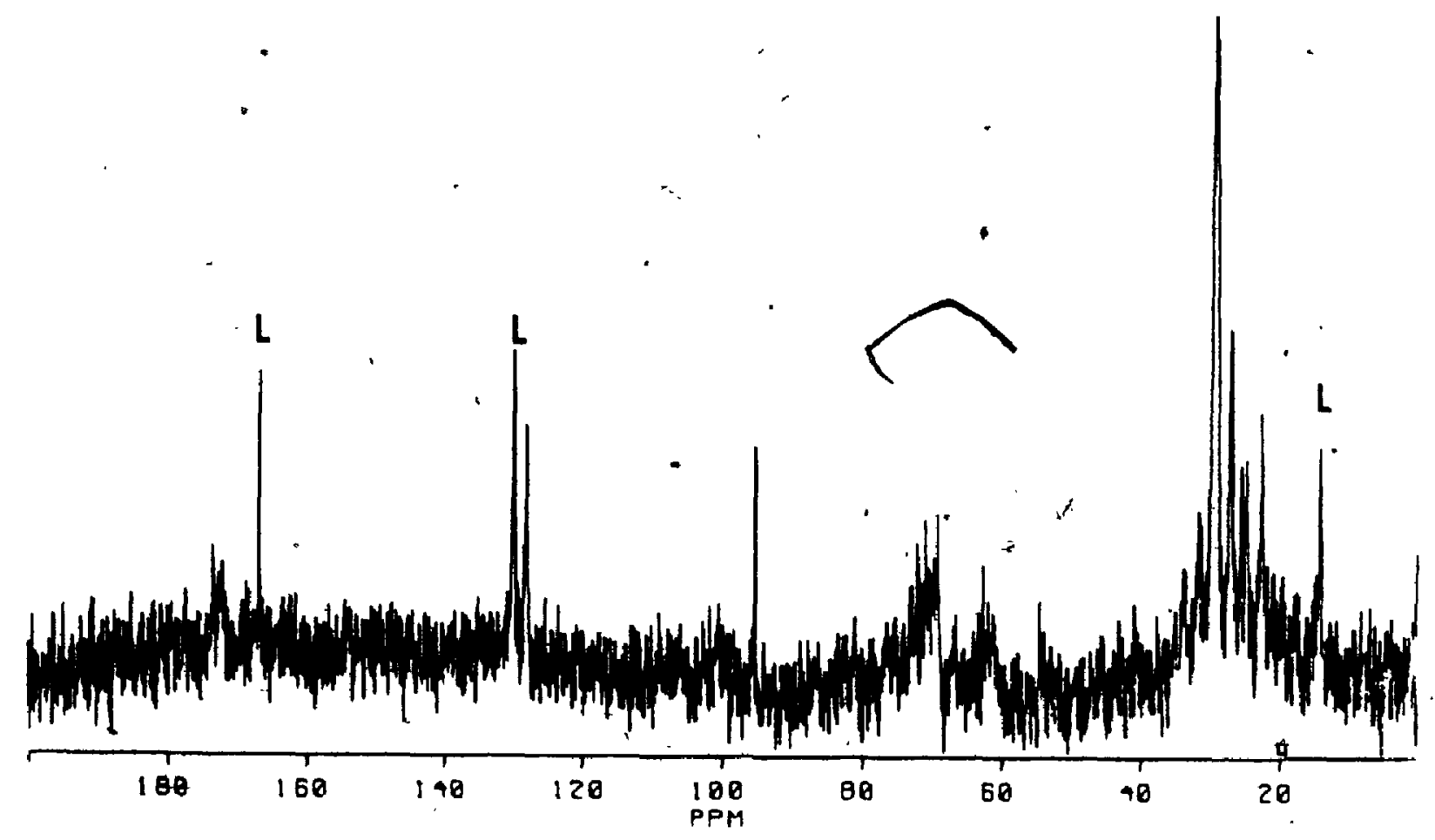

fig. $24{ }^{13} \mathrm{C}$ MMR ' opectrum of the precipitated protein from the mycelia of $\mathrm{E}_{2}$ culmorum HLX 1503

(FR-2). L lipid, associated with lipoprotein. 
The Second Experiment (FR-4)

The second experiment for the study of the degradation of butenolide $(F R-4)$ involved the addition, of $1,160 \mathrm{gm}$ of ${ }^{13} \mathrm{C}$-butenolide to the fermentor over a $24 \mathrm{~h}$ period starting at $30 \mathrm{~h}$. The enriched butenolide was added earlier than for FR-2 in order that all of it would be added over the 'crest of the butenolide concentration ( $f i g .21$ ) as it was determined in the first ${ }^{13} \mathrm{C}-2$ acetate fermentation experiment (GR-1). The fermentation analyzed for $170 \mathrm{~h}$, rather than the $98 \mathrm{~h}$ for FR-2, to allow the butenolide concentration to decrease longer. "Alternate $20 \mathrm{~mL}$ and $200 \mathrm{~mL}$ samples were taken at $12 \mathrm{~h}$ intervals. The 20 mL samples were taken at $25 \mathrm{~h}, 85 \mathrm{~h}, 11 \mathrm{~h}, 133 \mathrm{~h}$ and $158 \mathrm{~h}$ while the $200^{\circ} \mathrm{mL}$ samples were taken at $48 \mathrm{~h}, 72 \mathrm{~b}, 100 \mathrm{~h}, 122 \mathrm{~h}$ and $145 \mathrm{~h}$. The fermentor was harvested at $170 \mathrm{~h}$.

The $200 \mathrm{~mL}$ samples were used for butenolide and 3-ADON quantitation by HPLC and for NMR studies while the $20 \mathrm{~mL}$ samples were used just for HPLC quantitation of the levers of the toxins. Although the butenolide concentration did drop from the maximum of $29.4 \mathrm{mg} / \mathrm{L}$ to $4.4 \times \mathrm{mg} / \mathrm{L}$, it was not possible to elucidate any degradation products from the NMR data of the crude fungal extracts (fig. 25). 
fig. $25{ }^{13} \mathrm{C}$ NMR spectra of the crude fungal extracts from FR-4 at various times. The spectra are not plotted relative to each other so the peak intensities cannot be compared from spectrum." to spectrum. Note, however, the decrease in intensity of the butenolide resonances relative to the other peaks of the spectrum. which remain roughly constant.

B butenolide. 
A1 48 HOURS

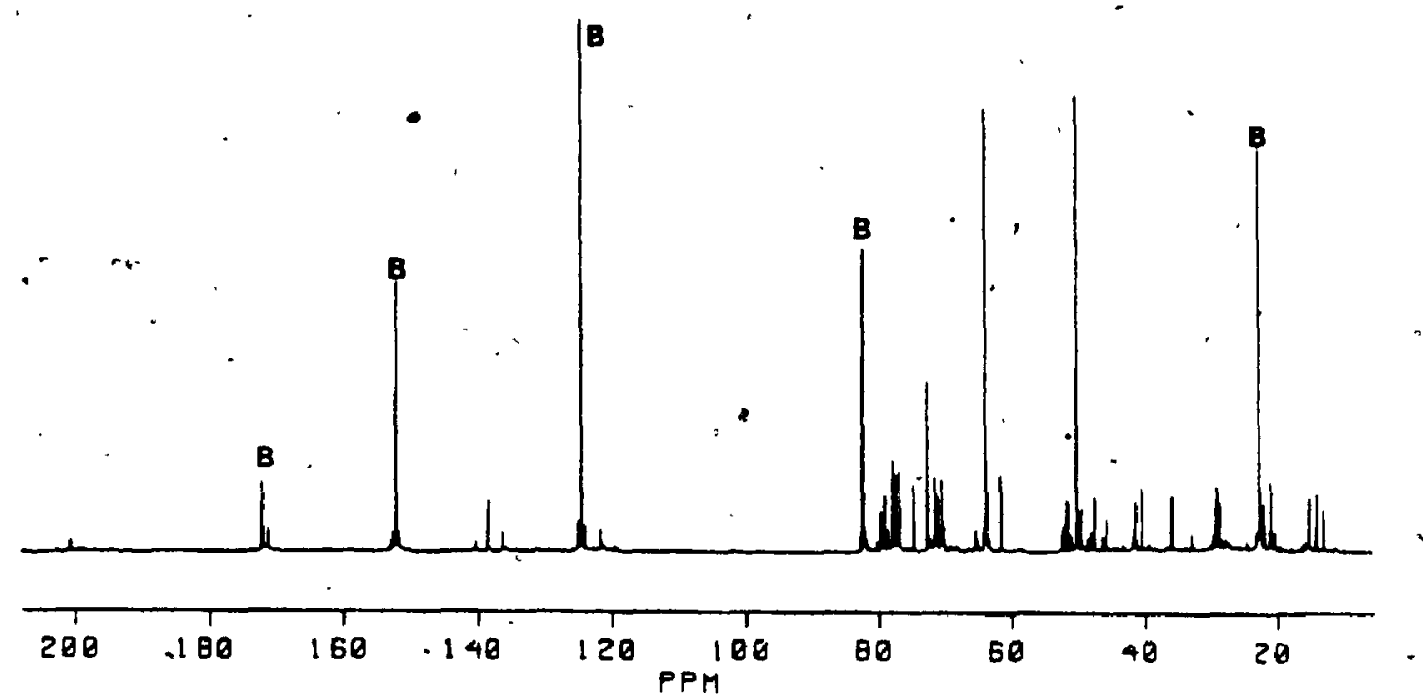

BI 100 HOURS

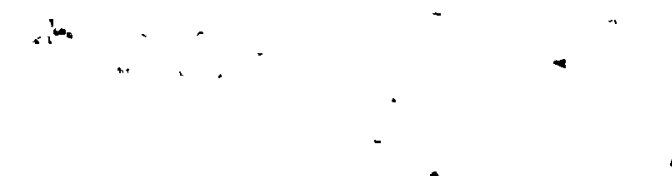

- $\therefore \quad \ldots$

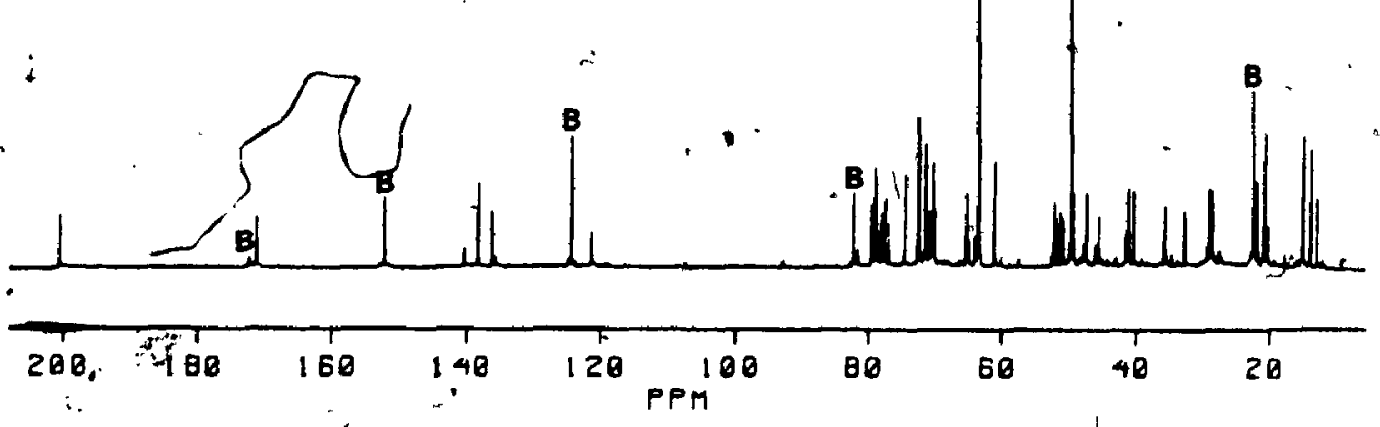

, C1, 145 HOURS

$\therefore$
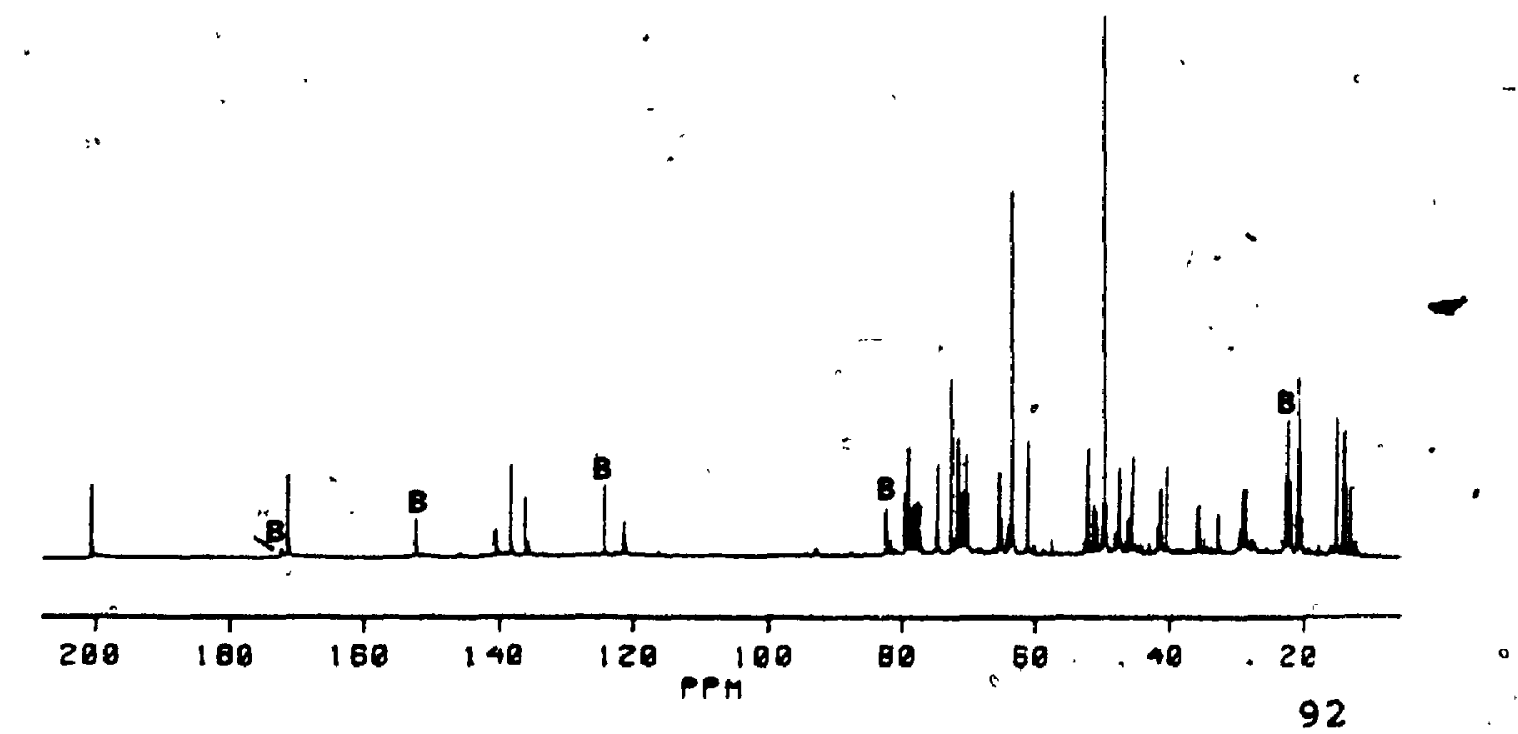
${ }^{13} \mathrm{C}$ NMR of Whole cells and an Aqueous Extract of the Whole cells from FR-4

More extensive studies were performed to try to determine the fate of the enriched butenolide which had been fed to the culture. ${ }^{13} \mathrm{C}$ NMR studies of the homogenized whole cells of FR-4 from $100 \mathrm{~h}$ and $170 \mathrm{~h}$ showed that there were no enriched compounds in the cellular components (fig. 26).. The only molecules to be observed in the whole cells were glycerol, mannitol and lipids.

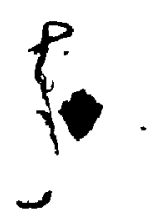




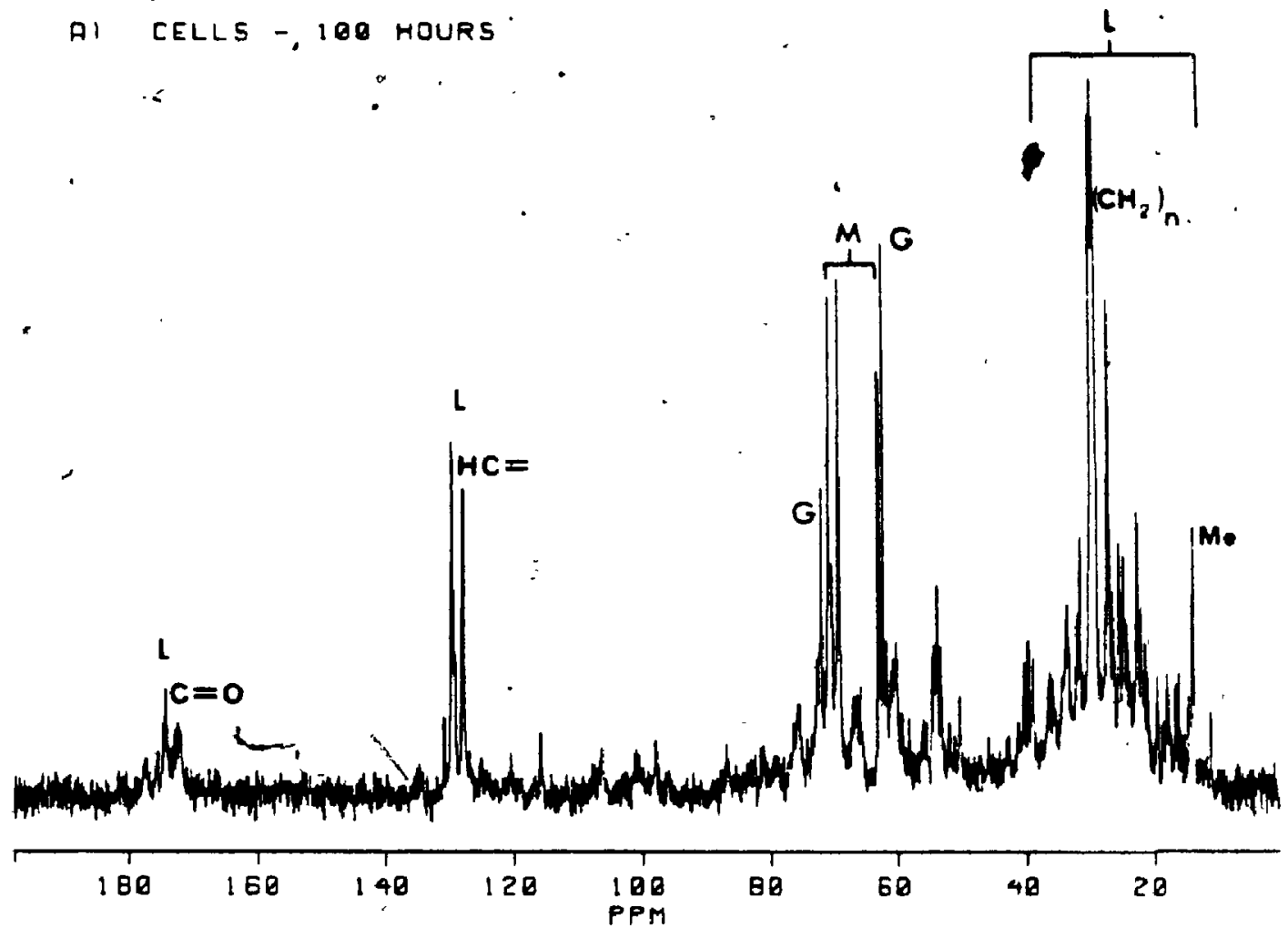

B) FINAL CELLS - 178 HOURS

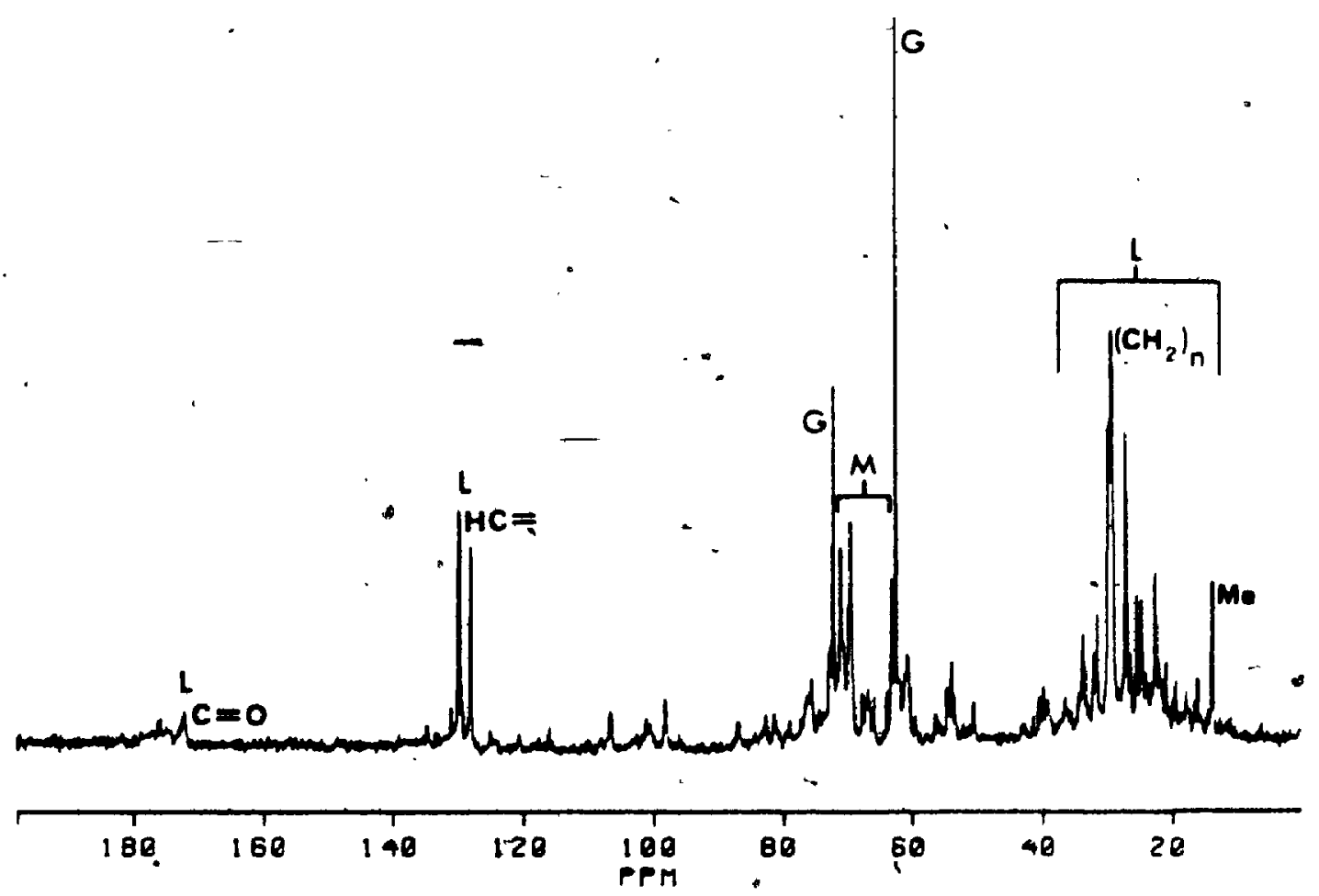

fig. $26{ }^{13} \mathrm{C}$ NMR spectra of homogenized whole cells from $F R-4$. Note the increase in the glycerol content of the cells. The 11pid and mannitc? concentrations remain relatively constant from $100 \mathrm{~h}$ to $170 \mathrm{~h}$. 
The aqueous extract of the harvested mycelia of $170 \mathrm{~h}$ from FR-4 showed that there was no enrichment in any of the water soluble cell components. Only glycerol and mannitol were observed in the ${ }^{13} \mathrm{C}$ NMR spectrum of the aqueous extract (fig. 27).

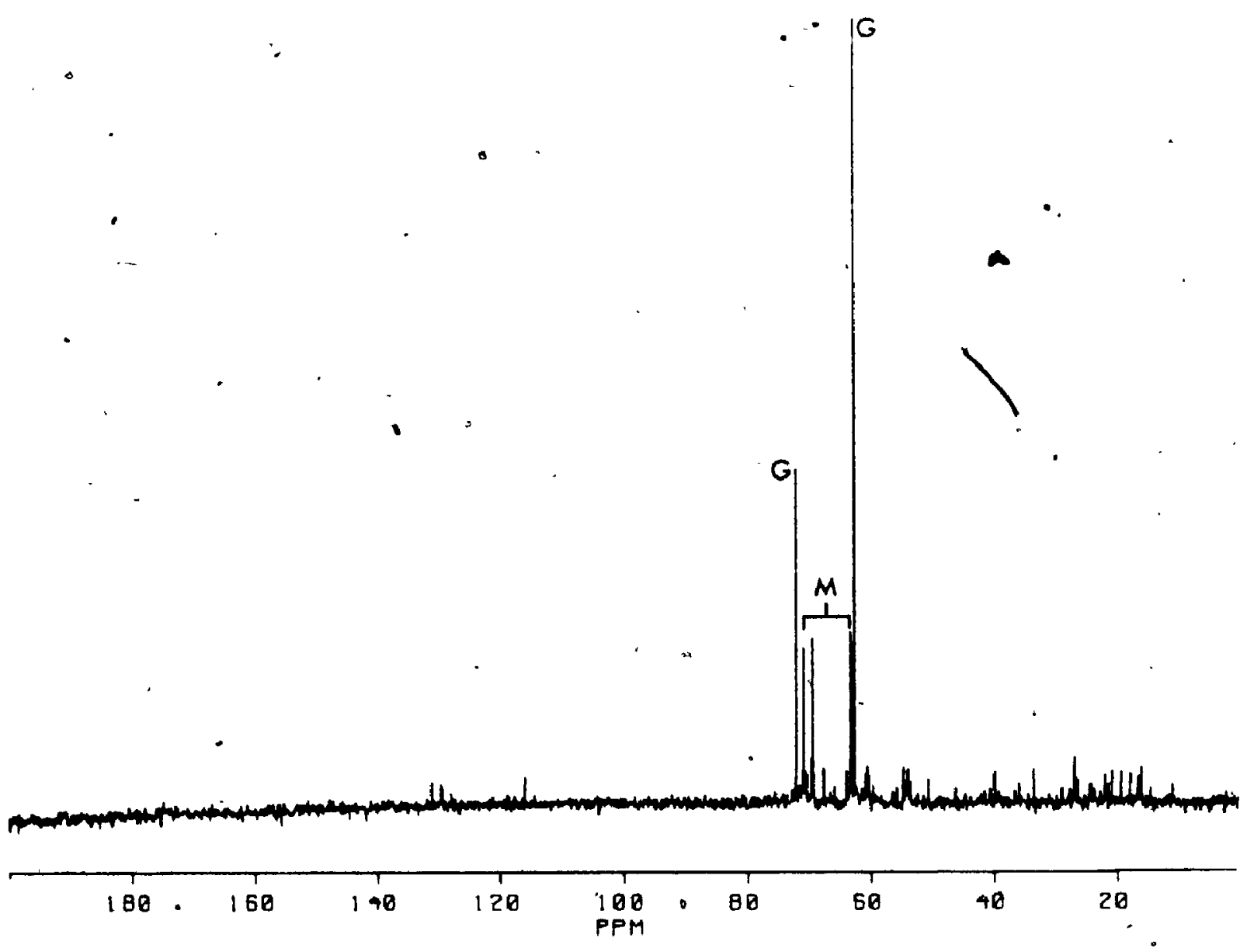

fig. $27{ }^{13} \mathrm{C}$ NMR spectrum of the aqueous extract from the final mycelia $(170 \mathrm{~h})$ of FR-4 . G glycerol, M mannitol.

In general, the results to the' degradation section. are Iffconclusive. No enriched products were observed in the medium, the whole cells, the aqueous extract of 
the, whole cells or in the precipitated proteins of cultures $t e$ which ${ }^{13} \mathrm{C}$ enriched butenolide had beef added. Therefore, from the evidence to date it cannot be determined what happeris to the butenolide (1). The only known fact is that the concentration of butenolide observed in the medium decreases after a maximum at about $36 \mathrm{~h}$ in the, $10 \mathrm{~L}$ stirred jar fermentation of E. cuImorum HLX 1503. 


\section{3-0 EXPERIMIENTAL TECHNIQUES}

3-1 General technique for shake flask experiments

All flasks were inoculated with Fusarium culmorum HLX 1503, as described by Miller and Blackwell (20), in $250 \mathrm{~mL}$ Erlenmeyer flasks, containing $50 \mathrm{~mL}$ of medium (unless stated otherwise). The fungal growth medium consisted of glucose $(20 \mathrm{~g})$, malt extract $(2 \mathrm{~g})$, peptone (2 g), $\mathrm{KH}_{2} \mathrm{PO}_{4}(2 \mathrm{~g}), \mathrm{MgSO}_{4} \cdot 7 \mathrm{H}_{2} \mathrm{O}\left(\begin{array}{ll}2 & \mathrm{~g}\end{array}\right)$, $\mathrm{FeSO}_{4} \cdot 7 \mathrm{H}_{2} \mathrm{O}(0.2 \mathrm{~g})$, and ammonium chloride $(3 \mathrm{~g})$ and adjusted to $\mathrm{pH}$ 6.3. All amounts are given per litre of distilled water (distilled water was put. through a Barnstead Nanopure II system; $18.3 \mathrm{M} \Omega \mathrm{cm}^{-1}$ ). The medium was autoclaved in the culture flasks prior to inoculation for 12 minutes at $121{ }^{\circ} \mathrm{C}$. After inoculation, the cultures were put on a rotary shaker $\left(3.81 \mathrm{~cm}\right.$ throw) at $220 \mathrm{rpm}$ at $25{ }^{\circ} \mathrm{C}$ for a minimum of $72 \mathrm{~h}$.

- After a minimum of $12 \mathrm{~h},{ }^{13} \mathrm{C}-1$ acetate, ${ }^{13} \mathrm{C}-2$ acetate, ${ }^{13} \mathrm{C}-5$ glutamic acid or ${ }^{15} \mathrm{~N}-\mathrm{glutamic}$ acid was added to the culture. Details of experiment duration and enrichment protocol may be found in the results section pertaining to particular experiments. All enriched compounds were dissolved in a minimum of. 
distilled water (water treated as above), without further purification as supplied from Merck sharp and Dome (MSD) isotopes, and added to the cultures under sterile conditions. The acetates were autoclaved in solution for 12 minutes at $121^{\circ} \mathrm{C}$ and the glutamic acids were dissolved in autoclaved ultrapure water and filter sterilized.

Cultures were harvested and filtered through tared" and dried whatman No. 1 filters: The mycelium was washed with water and dried under vacuum at $40^{\circ} \mathrm{C}$ to determine the dry weight. The culture filtrate from triplicate flasks was combined and analyzed unless described otherwise.

3-la Techniques for HPLC Quantitation of Butenolide 8 and 3-ADON, NMR Spectroscopy and Purification of ${ }^{13} \mathrm{C}$ Enriched Butenolide from Crude Fungal Extracts.

The combined filtrate,'(140 $\mathrm{mL}$ unless stated otherwise) was put.on a clinelut column which was then eluted six to eight times with $150 \mathrm{~mL}$ portions of ethyl acetate. The eluate was reduced to dryness using a rotoevaporator and redissolved up to $10 \mathrm{~mL}$ in methanol. A. 10 LL aliquot was injected on an HPLC (high performance liquid chromatography) Lichrosorb RP-18 
(C-18 reverse phase) column. The amount of butenolide and, 3-ADON was detected and quantitated using an ultraviolet detector set at $224 \mathrm{~nm}$. Quantitation was achieved by comparison with a standard of known butenolide and 3-ADON concentration (appendix 2).

The $10 \mathrm{~mL}$ methanol sample was then dried and redissolved in $\mathrm{C}^{2} \mathrm{HCl}_{3} \cdot{ }^{13} \mathrm{C}$ NMR spectroscopy at $62.8 \mathrm{MHz}$ (Bruker WM 250. NMR spectrometer) was performed to determine the degree of 1 chrichment in butenolide and 3-ADON. All samples within an individual experiment were redissolved to a constant volume of $\mathrm{C}_{2} \mathrm{HCl}_{3}$ to allow for the direct comparison of NiR spectra which enables the complex calculations to be performed on the da'ta. These calculations are outlined and described in appendix 1 :

Any purification of samples was performed on silica. gel '(Kieselgel 50; $0.063-0.200 \mathrm{~mm}, 70-230$ mesh ASTM) columns with the following solvent systems:

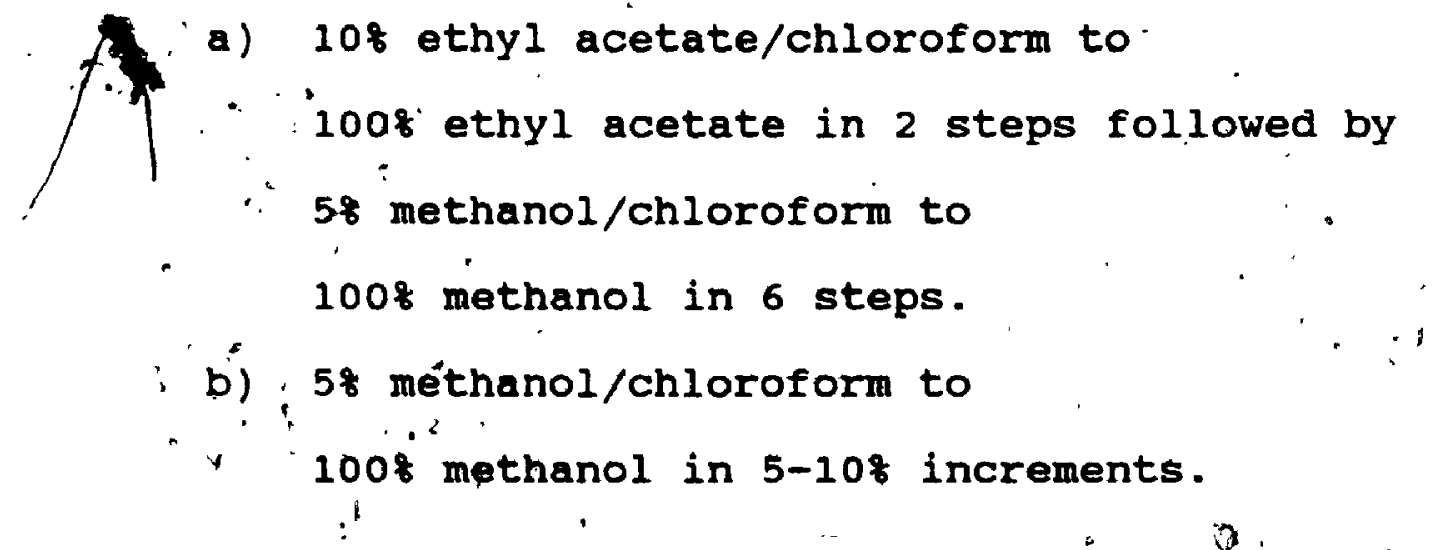


3-2 General procedure for stirred jar fermentor experiments.

Ten litres of production medium consisting of sucrose $(40 \mathrm{~g})$, glycerol' $(10 \mathrm{~g}),\left(\mathrm{NH}_{4}\right){ }_{2} \mathrm{HPO}_{4}(1 \mathrm{~g})$; $\mathrm{KH}_{2} \mathrm{PO}_{4}(3 \mathrm{~g}), \mathrm{MgSO}_{4} \cdot 7 \mathrm{H}_{2} \mathrm{O}(0.2 \mathrm{~g})$ and $\mathrm{NaCl}\left(\begin{array}{ll}5 & \mathrm{~g}\end{array}\right)$ (amounts are given per litre of ultrapure distilled water as above) was added, to a $15 \mathrm{~L}$ jar with $\mathrm{pH}$ (Ingold) and $\mathrm{O}_{2}$ electrodes (N.B. Scientific 900 series) and sterilized at $121^{\circ} \mathrm{C}$ for $1 \mathrm{~h}$. The medium was inoculated as described by Miller and Blackwell (20). The culture was grown at $28^{\circ} \mathrm{C}$; and was stirred at 400 rpm. Air was pumped into the fermentor at $250 \mathrm{~mL} \mathrm{min-1}^{-1}$ $\mathrm{L}^{-1}$ and the foam was controlled by a mechanical foam breaker. The initial pH of the culture was 6,3 which dropped to 4 over $24 \mathrm{~h}$ and was maintained at 4 by the addition of sterile sodium hydroxide solution $(0.8 \mathrm{M})$ throughout the fermentation. The protocol used "for the , addition of ${ }^{13} \mathrm{C}$-enriched species for each experiment may be found in the results section.

Samples (.200 mL unless otherwise stated) were withdrawn and filtered through a tared and dried whatman. No. $i^{*}$ filter. The filtrate $190 \mathrm{~mL}$ ) was collected and applied to a clinelut column while the 
mycelium was washed with distilled water, dried under vacuum at $40^{\circ} \mathrm{C}$ and the dry weight determined. The clinelut was eluted six to eight times with $200 \mathrm{~mL}$ aliquots of ethyl acetate. The eluate was reduced to dryness with a rotoevaporator and analyzed as above (see section 3-1a). A summary of the methodology and techniques used in the analysis of the metabolism of E. eulmerum HLX 1503 is given in figure 28 . 


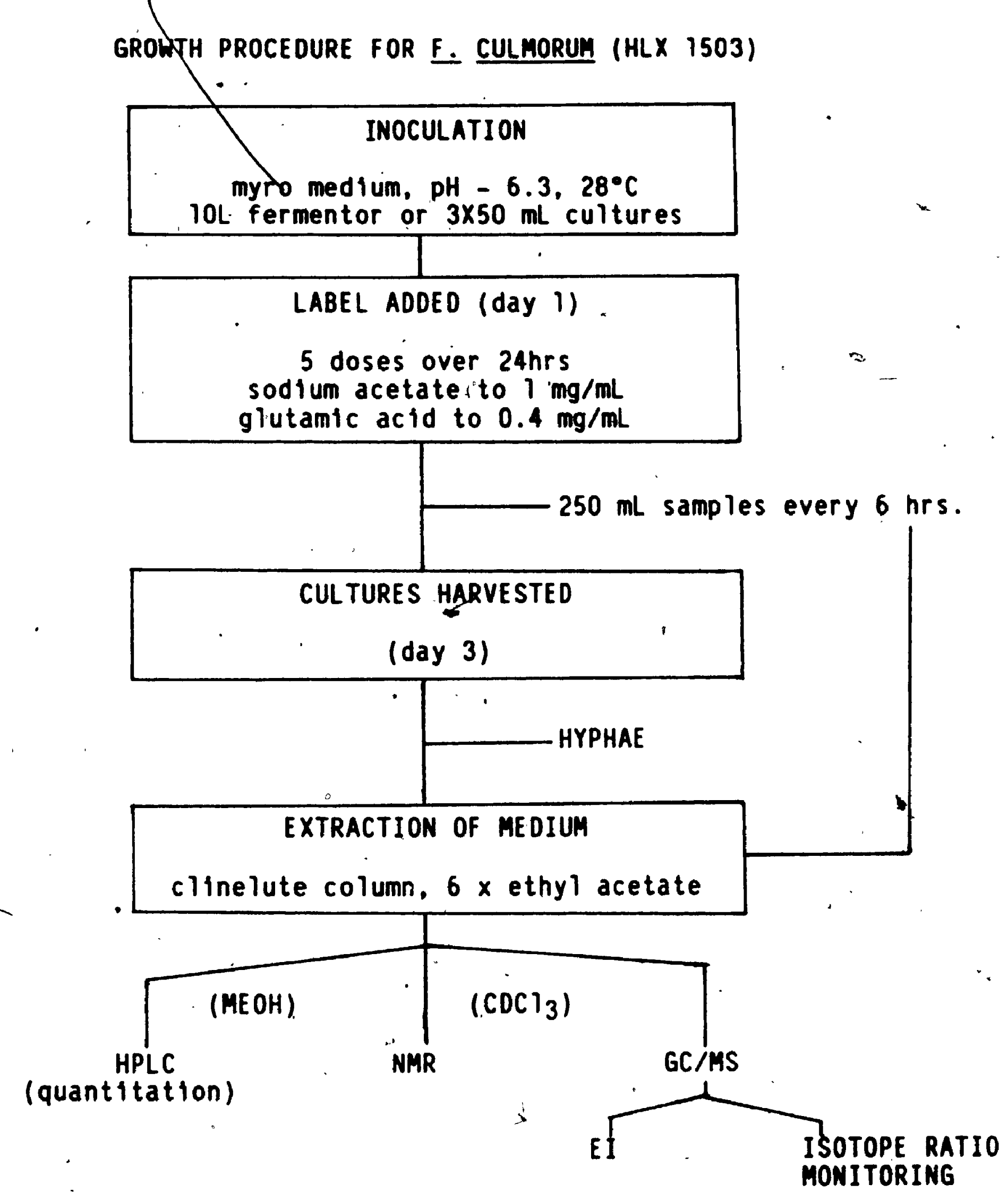

fig. 28 Experimental procedures for the growth of $\therefore$ F. culmorum (HLX 1503) and the analysis of 
3-3 Procedure for the precipitation of protein from mycelium.

The precipitation was performed on the mycelia from the second fermentation. experiment (FR-2) which was performed to determine degradation products of butenolide. Purified butenolide that had been produced in the first fermentation experiment (FR-1) and was ${ }^{13} \mathrm{C}$ senriched at several positions, was added to the fermentation. These mycelia (from FR-2) had been previously extracted and homogenized with $\mathrm{CH}_{2} \mathrm{Cl}_{2}$ to extract the $\mathrm{CH}_{2} \mathrm{Cl}_{2}$-soluble fatty acids and lipids. The frozen mycelia were thawed at room temperature and homogenized in 4 large centrifuge tubes with $0.61 \mathrm{~N}$ trichloroacetic acid (TCA) for 2 min with a Polytron. .

The homogenized hyphae were stored overnight in the TCA in the refrigerator. This mixture was then centrifuged at $3000 \mathrm{rpm}$ (Sorvall superspeed, RC2-B) for $20 \mathrm{~min}$ at 0 'c. The supernatant was collected and recentrifuged at $13,000 \mathrm{rpm}$ for $45 \mathrm{~min}$ at $0^{\circ} \mathrm{C}$. The supernatant from this centrifugation was decanted and the pellet was recentrifuged and washed with distilled $\mathrm{H}_{2} \mathrm{O}$. The pale grey precipitate was prepared for NMR analysis, with $\mathrm{D}_{2} \mathrm{O}$. 


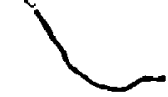

\section{3-4 Recovery Study}

$A^{*}$ number of preliminary studies were required to optimize and determine the accuracy of the procedures. The first experiment performed was a rovery study of butenolide from Clinelut columns. This was necessary to ascertain if anf correction was required on HPLC quantitation results and to determine if clinelut columns were a satisfactory method of extracting the media. The accuracy of the clinelut extraction was tested by a recovery study from samples spiked with a known concentration of the mycotoxin.' To determine the recovery, 3 samples containing $100 \mathrm{~mL}$ of modified Myro medium were spiked with $6 \mathrm{mg} / 100 \mathrm{~mL}, 12 \mathrm{mg} / 100 \mathrm{~mL}$ and 25 $\mathrm{mg} / 100 \mathrm{~mL}$ respectively. The modified Myro medium contains $\mathrm{NH}_{4} \cdot \mathrm{H}_{2} \mathrm{PO}_{4}(0.25 \mathrm{~g}), \mathrm{KH}_{2} \mathrm{PO}_{4}(1 \mathrm{~g}), \mathrm{NaCl}(2.5$ g), sucrose $(20 \mathrm{~g})$ and glycerol (5 g) made up to a volume of $500 \mathrm{~mL}$ and adjusted to a pH of 3.5. This modified Myro medium mimics the final composition and $\mathrm{pH}$ conditions of the growth medium at the end of fermentation. A $20^{\prime} \mathrm{mL}$ aliquot of each spiked sample was applied to a $20 \mathrm{~mL}$ clinelut column, worked up and analyzed as described in the experimental techniques for shake flask experiments section. Table 11 shows the recovery of each sample. 
Table 10. Recovery of butenolide from spiked media * samples.

\begin{tabular}{|c|c|c|}
\hline $\begin{array}{c}\text { sample } \\
\text { conc. spiked } \\
\mathrm{mg} / 100 \mathrm{~mL}\end{array}$ & $\begin{array}{c}\text { recovery } \\
\mathrm{mg} / 100 \mathrm{~mL}\end{array}$ & \\
\hline 6.0 & 6.1 & 101 \\
12.0 & 13.1 & 109 \\
25.0 & 24.9 & 100 \\
\hline
\end{tabular}

The high values for the recovery indicate that, within an experimental error of 10\%, all of the butenolide was recovered from the medium. Therefore, there is no need to correct the values obtained for the concentration of butenolide in any experiments involving extraction from clinelut columns. The $10 \%$ error is considered inherent in the HPLC quantitation technique. The accuracy of the quantitation is dependent on the injection. If the integration of, the injection is at the high end of the scale the calculations will give a low result whereas if the integration is low, the result will be a high quantitation. This is a factor which cannot be regulated and is therefore inherent in the technique. Also, a small variation at the level of the integration will cause a larger variation in the final result. The other major contributor to the possible erfors in the HPLC quantitation technique is the standard. The concentration of the standard wilin" increase slightly as 
it is kept due to solvent evaporation. To minimize this problem, the standard was kept sealed in the freezer and a fresh standard was made up every few experiments. The calculations required for the quantitation of butenolide and 3-ADON by HPLC are summarized in appendix 1.

The studies performed to determine the optimum conditions for culture, growth and ${ }_{i}^{3} \mathrm{C}$-enrichment. conditions are presented in the results and discussion section of this paper. 
Conclusion

This project was performed to study the metabolism of Eusarium culmorum HLX 1503 and the biosynthesis of butenolide; one of the secondary metabolites produced by this fungus. The relationship of butenolide production to the formation of the trichothecenes and the possible degradation 'of butenolide were also studied.

The conditions for the growth of $E_{\text {e culmerum }}$ HLX 1503 required to maximize butenolide production were determined in shake flask experiments and this data was later applied to $10 \mathrm{~L}$ stirred jar fermentor cultures. Increased oxygenation over the level of oxygenation required to maximize trichothecene formation was one of the requirements. The profile of butenolide and 3-ADON production was determined and it was shown that butenolide formation slowed as trichothecene formation increased.

The biosynthetic origin of butenolide was determined to be from the TCA cycle through glutamic acid. studies of the incorporation of ${ }^{13} \mathrm{C}-2$ acetate into butenolide and the resultant enrichment pattern were consistent with a product formed from the TCA cycle. The determination of glutamic acid as a precursor was 
accomplished by the addition of ${ }^{13} \mathrm{C}$ glutamic acid and ${ }^{15} \mathrm{~N}$ glutamic acid to the culture-medium of shake flask experiments. The increase in the yield of butenolide and the incorporation of the enriched glutamic acld into butenolide as observed in both ${ }^{13} \mathrm{C}$ and ${ }^{15} \mathrm{~N}$ NMR spectra were evidence that glutamic acid was definitely a metabolic precursor of butenolide.

Through the addition of the stable isotope ${ }^{13} \mathrm{C}-2$ acetate to the culture medium, we' were able to monitor the activity of the TCA cycle and the mevalonic acid pathway simultaneously. This was accomplished by analyzing the ${ }^{13} \mathrm{C}$ NMR spectra of the crude fungal extracts and the dynamics of the enrichment patterns of both'butenolide and 3-ADON. 3-ADON was studied as a representative trichothecene because it is the most abundarfily produced trichothecene by $E$. culmorum HLX 1503. The relationship of the production of each compound was studied and it was determined that butenolide formation is rapid earlidy in the fermentation than trichothecene formation. The formation of butenolide slowed down as the rate of - production of the trichothecenes increased. The production of secondary metabolites has been linked to nitrogen limitation. Butenolide production is initiated as the nitrogen source decreases and when the 
nitrogen is depleted, then trichothecene production is initiated.

The decline in butenolide concentration in the medium was first observed in our laboratory when the biosynthesis of $3-A D O N$ and other metabolites was being studied (20). It was observed in $10 \mathrm{~L}$ stirred jar fermentor studies and could not be duplicated in shake flask cultures. This possible degradation was studied through the addition of ${ }^{13} \mathrm{C}$ enriched butenolide to the culture medium of a 10 L stirred. jar fermentation experiment but although the concentration of butenolide did decline, no products were observed to incorporate the ${ }^{13} \mathrm{C}$ from the enriched butenolide. Therefore, no. conclusions could be drawn as to the possible degradation of butenolide or reasons for this degradation. One hypothesis is that the fungus uses the butenolide that is produced as a nitrogen source later in growth. 
Suggestions for Future Work

There are several projects which could be pursued to continue the work of this study:

1) The investigation of the degradation of butenolide could be continued to discover and characterize possible degradation products. It is possible that butenolide is used as a nitrogen source for the fungus since it is produced as the nitrogen source in the medium becomes limiting. once all of the exogenous nitrogen is exhausted and trichothecene production is initiated, the butenolide concentration in the medium decreases. This lead to the hypothesis that butenolide may be used as a nitrogen source, when all other nitrogen sources are depleted.

2) A study of the biosynthetic pathway Iram glutamic acid to butenolide to prove the route proposed in this study. This could be accomplished by searching for enriched intermediates at the times: in the. fermentation when butenolide production was increasing. The intermediates could'also be sýnthesized and analyzed to attempt to mimic the proposed route. The synthesized intermediates could, be used to obtain. structural data (ie. NMR data, HPLC characteristics, UV 
and IR data etc.) to help in the characterization of any possible intermediates.

3) Investigation of the role of 4-acetamido-2butenoic acid discovered by Vesonder et al. (19) in the biosynthesis of butenolide. This could be accomplishea by purifying the compound from the fungal extract and analyzing for ${ }^{13} \mathrm{C}$ enrichment patterns similar to butenolide.

4) The chirality of butenolide is still unresolved. As it is unlikely that both $a$ and $L$ enantiomers are produced biologically, the molecule should have only one racemate which can be determined by NMR spectroscopy with a chiral shift reagent. It is also unlikely that the molecule racemizes in solution as this would involve the opening and reclosure of the lactone ring. X-ray crystallography can also be used to determine the absolute steriochemistry if only one enantiomer is present. 
References

1). Richard, J.L., Thurston, J.R. (eds.) (1986) In Diagnesis of Mycotoxicoses." Martinus Nijhoff Publishers, Boston.

2) Smith, J.E. and Moss, M.O. (1985) In Mycotoxins. John Wiley \& Sons, Toronto.

3) Scott, P.M. (1985) In Mycotoxins: A canadian Perspective, NRC Publication, ISBN 0316-0114

4) Steyn, P. (1980) In The Biesynthesis of Mycotoxins: A Study in Secondary Metabelisme" (ed. Steyn, P.) Academic Press, Toronto.

5) Mann, J. (1980) In Secondary Metabolism. (eds. Atkins, clarendon Press, oxford.

6) Luckner, M. (1984) Secondary Metabolism in

- Microorganisms, plants and Animals. "SpringerVerlag, New York.

7) Bu'Lock, J.D. (1980) In The Biosynthesis of Mycotoxins: A Study in Secondary Metabolism (ed. P.S. Steyn) pp. 1-16. Academic Press, Totonto.

8) Booth, C. (1984) In The Applied Mycelogy of Fusartum. (ed. Moss, M.O. and Smith, J.E.) pp 1-5. Cambridge University Press, New York.

9) Marasas," W.F.O., Nelson; P.E. and Toussoun, T.A. (1984) Toxigenic Pusarium species: Identity and Mycotoxicology. The Pennifyivania state Unfversity Press, University Park.

10) Marasas, W.F.O. and Nelson, P.E. (1987) dycetoxicelegy pp. 42-44. The Pennsylvania University. Press, University Park.

11) Trenholm, H.L. et al. (1981) Survey of Vomitoxin Contamination of the 1980 White winter Wheat crop in Ontario, Canada. JAOCS, Dec.: 992A-994A.

12). Ueno, Y. (1978) In Mycotoxins: In Human and Animal Health. (ed. Ridricks, J.V., Hesseltine, C.W. and Mehlman, M.A.) pp. 189-207. Pathotox Pub. Inc., Illinois. 
13) Yoshizawa, T. (1983) In Trichothececnes: Chemicale Bieleaical and Toxtcological Aspectse (ed. Ueno, y.) pp. 60-73. Elsevier, New York

14) Joffe, A.z. (1971) In Microbial Toxins Vol. VII, (eds..s. Kadis et al) pp. 139-187. Academic Press, N.Y.

15) Cane, D.E., Swanson, S., Murthy, P.P.N. (1981) Trichodiene. Biosynthesis and the Enzymatic Cyclization of Farnesyl Pyrophosphate. J.A. Chem. Soc. 103, 2136-2138.

16)' Yates, S.G. (1971) In Microbial Toxins Vol VII.

- (ed. Kadis, S. et al.). pp 191-205. Academic Press, New York.

17) Garner, G.B. and Cornell, C.N. (1977) In Mycotoxic Eungi: Mycotoxins, Mycotoxiceses Vol. 2, (eds. Wyllie, T.D. and Morehouse, L.G.). PP. 45-62. Dekker Inc., New York.

18) Grove, M.D. et al. (1970) Mycotoxins Produced by Eusarium tricinctum as Possible causes of Cattle Disease. J. Agr. Food Chem. . 18, 734-736.

19) Vesonder, R.F., et al., (1977) 4-Acetamido-2-butenoic Acid from Eusarium graminearum. Phytochemistry, 16. 1296-1297.

20) Miller, J.D, and Blackwell, B.A. (1986) Biosynthesis of 3-acetyldeoxynivalenol and other metabolites by Eutartum culmorum HLX $1503^{\circ}$ in a stirred jar fermentor. Can. J."Bot. 64, 1-5.

21) Vederas, J.C. (1987) The Use of Stable Isotopes in Biosynthetis studies. Nat. Prod. Rep. 4, 277-337.

22) Williamson, J.M. et al. (1985) Biosynthesis of the B-Lactain Aptibiotic, Thienamycin, by Stréptomyces cattileya. J. Biol. Chem., 260, 4637.

23) Metzier; D.E. (1977) In Biochemistry: The chemical Reactions of Iivina cells, pp. 649-651, Academic Press, New York.

24) Blackwell, B.A. .:et al: (1985) ${ }^{13} \mathrm{C}$ NMR study of -the Biosynthesis of Toxins by Eusarium graminearum:" J. Biol. Chem. 260, 4243-4247. 
25) Hor, A. and Shimizu, Y. (1983) Biosynthetic ${ }^{15} \mathrm{~N}-$ Enrichment and $15_{N}$ NMR Spectra of Neosaxitoxin and Gonyautoxin-II: Application to structure Determination. J. Chem. Soc., Chem., Comm., 790.

26) Martin, F. (1985) $15 \mathrm{~N}$-NMR Studies of Nitrogen Assimilation and Amino Acid Biosynthesis in the Eotomycorrhizal Fungus cenococcum graniforme. FEBS Lett., 183, 350 .

27) Morris, G.A. and Freeman, R. (1979) J. Am. Chem. Soc., 101, 760 .

28) vo Philipsborn, W. and Muller, R. (1986) ${ }^{15}$ N-NMR Spectroscopy - New Methods and Applications. Angewandte Cherie, 25, 383-486. 


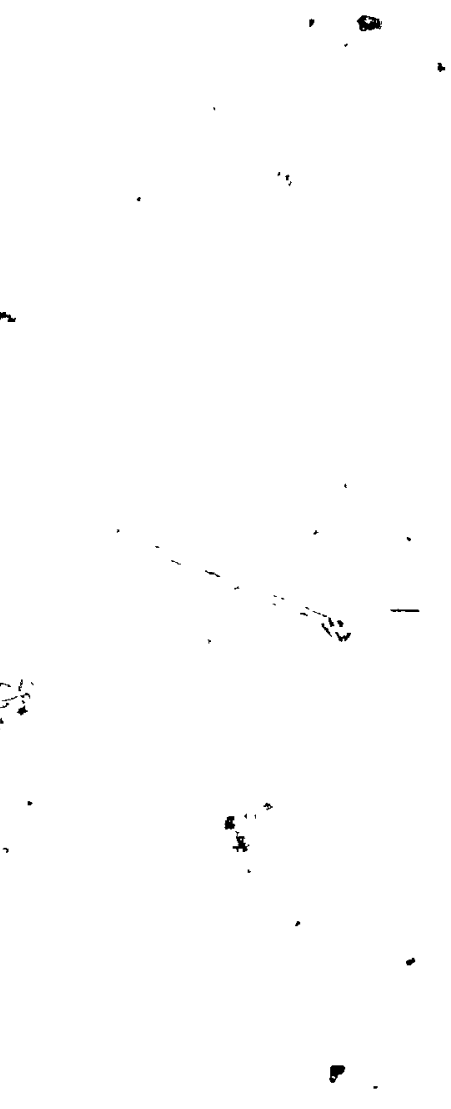

Appendix 1

Calculations Used in the Analysis of ${ }^{13} \mathrm{C}$ NMR Spectral Data
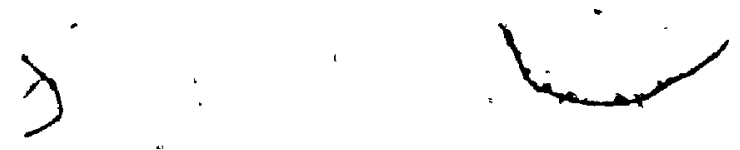


\section{Appendix 1}

Calculations Used in the Analysis of ${ }^{13} \mathrm{C}$ NMR Spectral Data Obtained from 10 L stirred Jar Fermentor Experiments

Crude extracts of fungal growth media from fermentation experiments have several characteristics which permit in depth analyses through the use of NMR spectroscopy. However, several conditions must first be fulfilled before the analysis can be completed and be considered valid.

First, all extracts from the same experiment must be prepared in the same manner keeping the final volume of the sample constant. The crude fungal extract is, therefore, redissolved in the NMR solvent (usually $\mathrm{CDCl}_{3}$ ) to a constant final volume without consideration for the amount of extract initially available. Samples taken earlier in the fermentation will generally have less extractable material than those taken later in the fermentation. Therefore, redissolving the samples to a constant volume and obtaining the ${ }^{13} \mathrm{C}$ NMR spectra essentially gives a picture of the metabolism of the cell at that time as it is represented by the ethyl acetate extractable metabolites. The 'picture' becomes further defined when ${ }^{13} \mathrm{C}$ acetate is added to the growth 
medium and becomes incorporated into the metabolites. Metabolites of different metabolic pathways acquire different enrichment patterns. The dynamics of this enrichment and the relationship to the biosynthesis of the metabolites is the purpose of the studies with ${ }^{13} \mathrm{C}$ NMR spectrosciopy.

A second very important condition which must be satisfied is that the acquisition parameters of the spectra must remain constant for an experimental series. In order that the spectra obtained in the studies described may be comparable, the spectra from all of the fermentation experiments and the standard unenriched. spectrum were acquired under the same conditions. The acquisition time, was 0.5 seconds with a relaxation time of 2.5 seconds. Therefore, the total recycle time was 3.0 seconds. The sweep width was 15 $\mathrm{kHz}$ and the pulse width was 8.0 which corresponds to a 60 pulse. It is not important to keep the number of scans constant because they determine the signal to noise ratio and do not affect the relative integrations of each peak. However, for continuity within a fermentation experiment, the number of scans was maintained at either 10,000 or 8,000 scans for each spectrum. The standard unenriched spectrum was acquired with 8,000 scans. 
The ${ }^{13} \mathrm{C}$ Ręsonances and Their Use in This Study

In ${ }^{13} \mathrm{C}$ NMR spectroscopy single resonances occur for the ${ }^{13} \mathrm{C}$ carbon atoms at specific frequencies depending on the type of carbon atom detected. These resonances will be, split into a doublet with a unique coupling constant (or $\mathrm{J}$ value) when a neighbouring carbon atom is also a ${ }^{13} \mathrm{C}$. This splitting of the resonance into a doublet producespa'characteristic of the carbon NMR spectra: the friplet. Contrary to proton spectra where a triplet indicates a proton. split, by 2 neighbouring protons, the triplet in ${ }^{13} \mathrm{C}$ spectra signifies the carbon atom itself as well as the carbon. splite by a neighbouring ${ }^{13} \mathrm{C}$. The centre resonance arises from the ${ }^{13} \mathrm{C}$ atom next to $\mathrm{a}^{12} \mathrm{C}$ atom and the two ${ }^{13} \mathrm{C}$ satellites indicate the ${ }^{13} \mathrm{C}$ atom next to one other ${ }^{13} \mathrm{C}$ atom. If a ${ }^{13} \mathrm{C}$ atom is surrounded on two sides with ${ }^{13} \mathrm{C}$ carbon atoms, then a multiplet will occur. If the coupling constants of each of the carbons differ sufficiently then the multiplet will be defined enough that all 9 resonançes may be determined. The 9 resonances arise from all of the possible combinations of the ${ }^{13} \mathrm{C}$ next to two ${ }^{12} \mathrm{C}^{\prime} \mathrm{s},{ }^{13} \mathrm{C}$ adjacent to one ${ }^{12} \mathrm{C}$ and" one ${ }^{13} \mathrm{C}$ and the ${ }^{13} \mathrm{C}$ neighbouring two ${ }^{13} \mathrm{C}$ atoms. This is true of the ${ }^{13} \mathrm{C}$ enriched butenolide obtained 
when ${ }^{13} \mathrm{C}-2$ acetate is incorporated because the three adjacent carbons in the lactone ring become enriched.
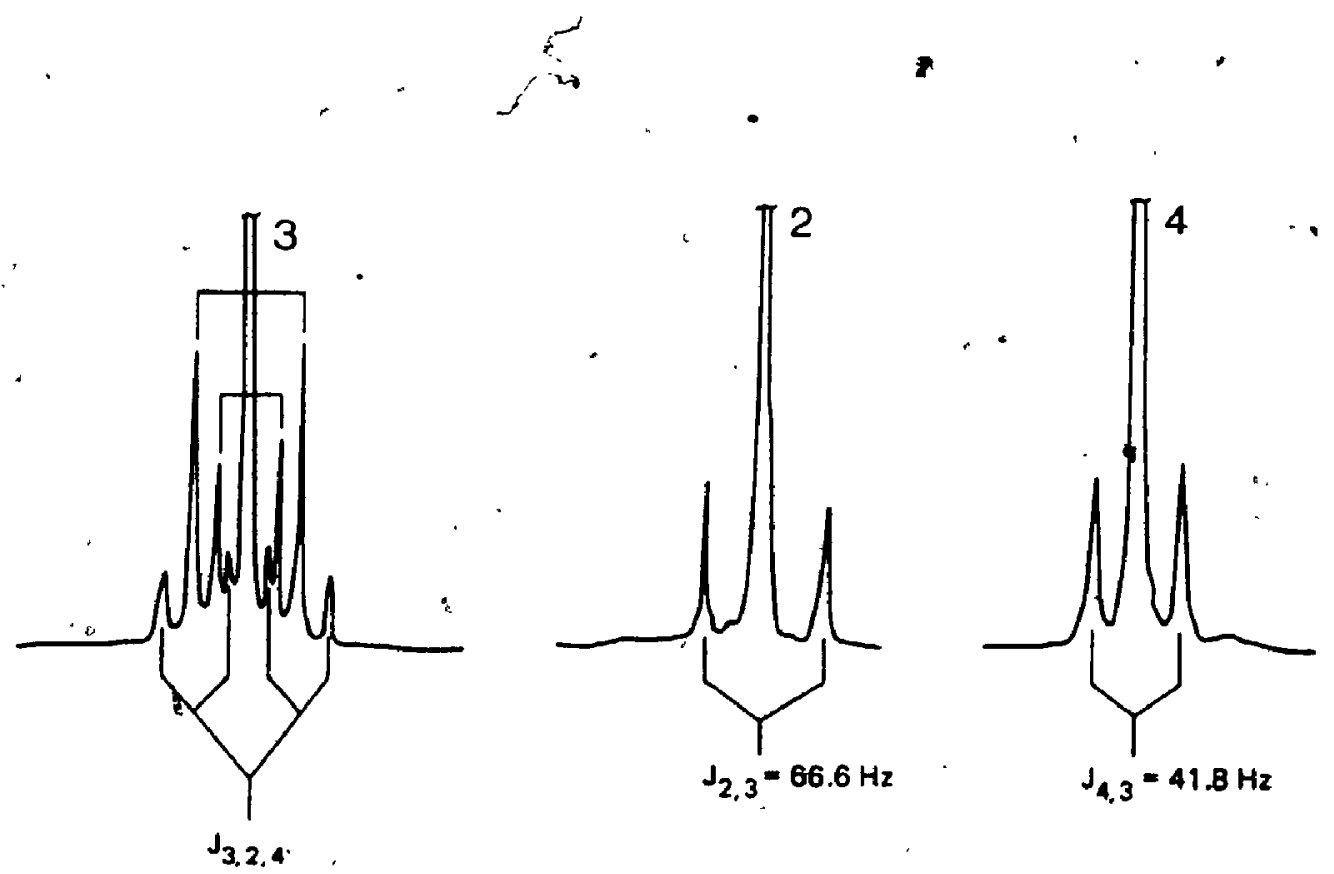<smiles></smiles>

fig. At The resonances of $\mathrm{C}-2, \mathrm{C}-3$ and $\mathrm{C}-4$ of butenoIide with the coupling constants indicating which satellites are associated with each carbon atom.

calculation of Total ${ }^{13} \mathrm{C}$ Enrichment at a specific: Carbon Atom

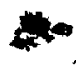

The total enrichment at a specific carbon atom is calculated by comparing the intensities of the unenriched butenolide standard spectrum with the 
$\pi$

enriched sample spectrum. The carbonyl carbon resonances of butenolide, which are not enriched when ${ }^{13} \mathrm{C}-2$ acetate is added to the fermentation, are used to compare the two spectra to each other. The ratios of the intensity of each carbon atom $(c-2, c-3, c-4$ and the AC-Me) to the carbonyl carbon within the standard spectrum are determined. From these ratios, the unenriched intensities of the enriched sample resonances can be calculated by multiplying the standard intensity ratio of each carbon atom by the average intensity of the butenolide carbonyl carbons in the sample spectrum. The total enrlchment at the specifis carbon atom is determined by dividing the sum of the intensities of the centre peak and the satelites by the calculated intensity. The calculations were carried out as follows:

Let $B 2, B 3$ and $B 4$ represent the intensity of the $\mathrm{BA}$ carbon atoms $2,3,4$ and the acetate methyl group respectjvely of the standard butenolide $13 \mathrm{C}$ NMR spectrum.

$\mathrm{BC}$

represent the average of the intensities of the two carbonyl carbons of the standard spectrum.

S2C, S3C, S4C represent the calculated SAC intensities of the sample butenolide.

$S 2 n, S 3 n, S 4 n$ represent the actual NMR SAn intensities of these carbon atoms using only the centre peak. 
and TE2, TE3, TE4 TEA

represent the total or overali enrichment at carbon atoms $\mathrm{C}-2$, $\mathrm{C}-3, \mathrm{C}-4$ and the acetate methyl group respectively.

To determine the unenriched intensity of the sample spectra:

$$
\begin{aligned}
1, s 2 C & =\frac{B 2}{B C} \times s C \\
s 3 C & =\frac{B 3}{B C} \times s C \\
S 4 C & =\frac{B 4}{B C} \times S C \\
S A C & =\frac{B A}{B C} \times S C
\end{aligned}
$$

where B2, B3, B4, BC and SC are obtained from the ${ }^{13} \mathrm{C}$ NMR spectra'l data.

From the unenriched intensity of each carbon the enrichment at each carbon atom was calculated:

$$
\begin{aligned}
& =T E 2=\frac{52 n}{S 2 C} \quad \text { TE3 }=\frac{510}{83 c} \\
& \text { TE4 }=\frac{\text { S4R }}{\text { S4C }} \quad \text { TEA }=\frac{\text { SA }}{\text { SAC }}
\end{aligned}
$$

where s2n,'s3n, s4n and sAn are obtained from the ${ }^{13} \mathrm{C}$ MMR spectral data of the crude fungal extract.

Calculation of the Ratio of single to Multiple Enrichment in Butenolide 
The ratio of the single to multiple enrichment is determined by dividing the sum of the satellites of a particular resonance by the sum of the centre resonance. plus the satellites. This calculation is possible because in ${ }^{13} \mathrm{C}$ NMR, spectra the ${ }^{13} \mathrm{C}$ satellites indicate the contribution from multiple enrichment and arise only from the ${ }^{13} \mathrm{C}$ carbon atoms that have a neighbouring ${ }^{13} \mathrm{C}$ carbon.

$\underset{\text { Enrichment }}{\text { Fraction of Multiple }}=\frac{\text { Sum of the Satellites }}{\text { Total (centre + satellites) }}$

Since the resonances that arise from triple and double enrichment are completely resolved at $\mathrm{C}-3$ of butenolide (fig. Al), the ratio of triple to double enrichment can also be calculated. $"$ This is accomplished by dividing the sum of the satellites arising from triple enrichment' only, by the sum of the satellites arising the double enriched species. The origin of each set of satellites can be determined by matching the coupling constants ( $J$ values) with the observed coupling constants of $\mathrm{C}-2$ and $\mathrm{C}-4$.

\footnotetext{
Ratio: Triple to = Satellites from $c-2$ \& $c-4$ couplings Double Enrichment sat. from C-2 PLUS Sat. from $\mathrm{C}-4$ 
Per cent of Triple Enrichment in Butenolide as Determined by $\dot{c}-3$ splitting Pattern Intensities

The determination of the per cent of triple enrichment is similar to the calculation for the ratio of multiple to single enrichment except that these calculations can only be performed using the resolved satellites of the $c-3$ resonances. $\frac{\text { - Triple }}{\text { Enrichment }}=\frac{\text { Sum of sat from Triple Enrichment }}{\text { Total at } c-3 \text { (centre plus all sat.) }} \times 100$

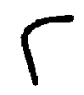

Extent of Enrichment in 3-ADON

The extent of enrichment in the trichothecene, 3-ADON (3), is monitored by comparing the resonance from $\mathrm{C}-2$ and $\mathrm{C}-3$ (fig. $\mathrm{A} 2$ ). When $13 \hat{\mathrm{C}}-2$ acetate is incorporated into 3-ADON, the carbon at $\mathrm{C}-2$ becomes enriched whereas the carbon at $c-3$ remains unenriched. These two carbons have. the same intensity when not enriched so to determine the extent of enrichment, the intensity of $\mathrm{c}-2$ is divided by the intensity of $\mathrm{c}-3$.

Extent of Enrichment $=\frac{\text { intensity of } \mathrm{C}-2}{\text { in 3-ADON }}$
intensity of $\mathrm{C}-3$

By calculating the extent of enrichment in 3 -ADON, the incorporation of ${ }^{13} \mathrm{C}-2$ acetate into both butenolide 
and the trichothecenes can be monitored simultaneously and the dynamics of their metabolic relationship can be" followed.

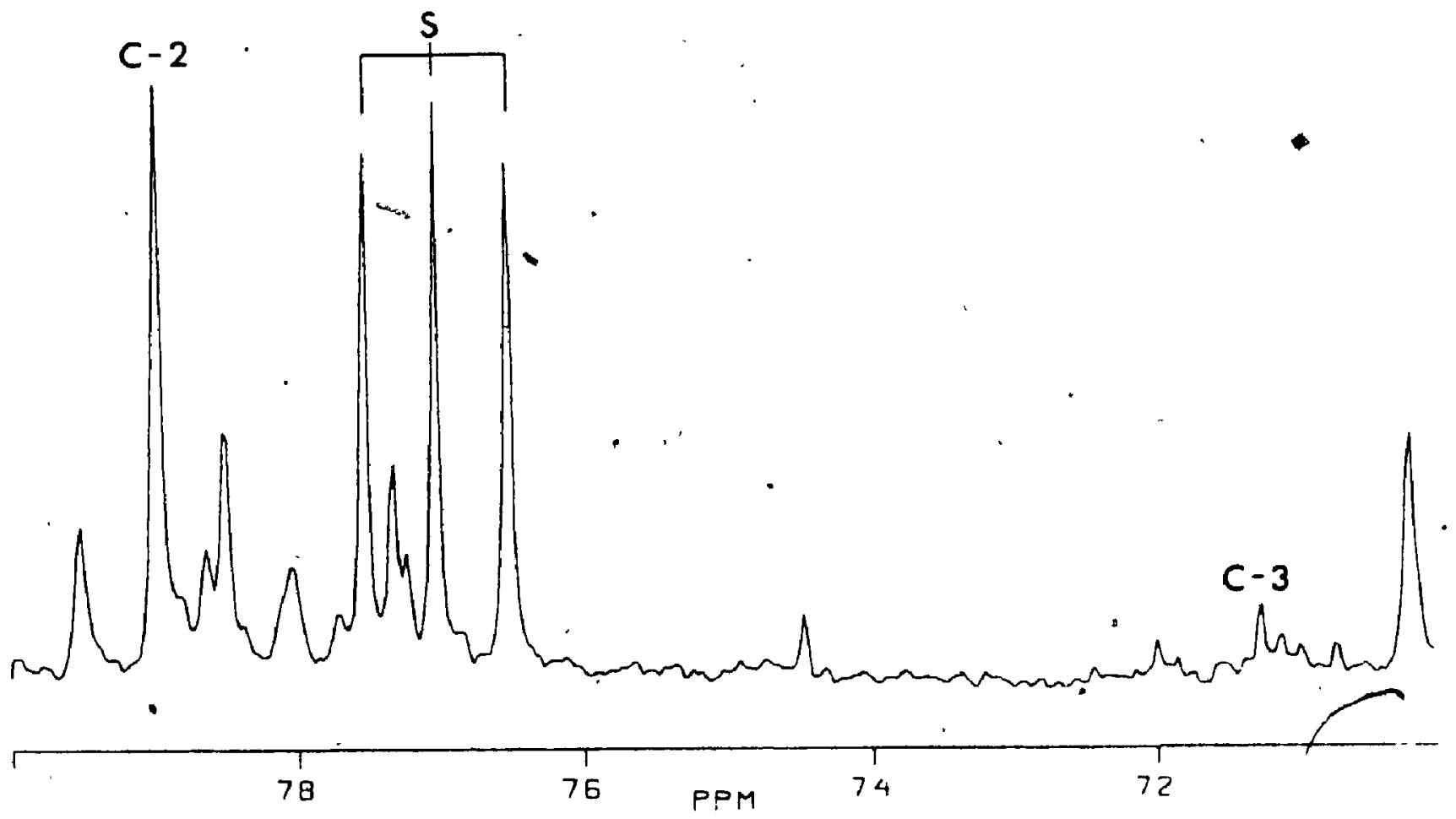

fig. A2 Expansion of the region of the ${ }^{13} \mathrm{C}$ NMR spectrum and the resonances used to determine $\rightarrow$ the level of enrichment in 3-ADON. 


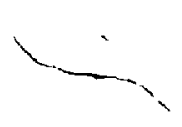

3

MPLC Quantitation Calculations 


\section{Appendix 2}

Metabolite Quantitation-by High Performance Liquid Chromatography (HPLC)

The quantitations of 3-ADON and butenolide from the crude extracts of the fungal medium were performed using a prepacked Lichrosorb RP-18 $(\dot{5} \mu \mathrm{m}){ }_{3}^{25} \mathrm{~cm}$ column with an internal diameter of $4.00 \mathrm{~mm}$. The metabolites of interest were detected by" ultraviolet light at $224 \mathrm{~nm}$ and compared with known' standards.

standards were prepared in distilled in glass methanol with 3-ADON concentration being double that of the butenolide concentration. The crude fungal extracts were always redissolved in distilled in glass methanol to a final volume of $10 \mathrm{~mL}$. The amount of mycotoxin, either butenolide or 3-ADON, in the injection was determined as follows: for a $10 \mathrm{\mu L}$ injection of both the standard and the sample

integration of the toxin integration of the standard $x \mathrm{~A} \mu \mathrm{g}$ in standard $=\mathrm{X} \mu \mathrm{g}$ toxin in injection

In some cases, the extract was too concentrated so the sample injection was decreased by a factor of 2 . Therefore, the value obtained for the $\mu g$ of toxin in the injection was multiplied by two. 
once the amount of the metabolite in the injection was determined, it was necessary to calculate how much would then be in the extracted culture medium. This was accomplished by incorporating all of the dilution factors as follows:

$x \mu g$ toxin in $=x \mathrm{mg}$ toxin in

$10 \mathrm{\mu L}$ injection $10 \mathrm{~mL}$ of redissolved extract

But, the redissolved extract was initially from $20 \mathrm{~mL}$ of culture medium. 'Therefore:

$\frac{x m a \text { toxin }}{20 \cdot m L \text { medium }}=Y m g$ of toxin per $m L$ of extracted medium b

Multiplying $Y$ by 1000 gives the final concentration of the toxin, either butenolide or 3-ADON, in the desired-mg per I of culture medium.

Example: from Recovery sţudy.

. $356788 \times 2.68 \mu g$ butenolide $=1.21 \mu g$ bf butenolide 788072 in standard in injection

$=1.21 \mathrm{mg}$ in $10 \mathrm{~mL}$ redissolved extract

But originally in $20 \mathrm{~mL}$ of culture medium $1.21 \mathrm{mg} / 20 \mathrm{~mL}$ of medium $=0.06066 \mathrm{mg}$ per $\mathrm{mL}$ of medium $\therefore=60.9 \mathrm{mg}$ per I 


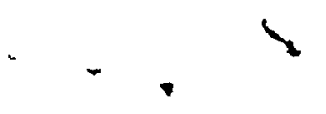

:

Appendix 3

Isotope Ratio Monitoring

$\rightarrow$ 


$$
\text { / Appendix } 3_{4}+:=
$$

${ }^{13} \mathrm{C} /{ }^{12} \mathrm{C}$ ' Isotope Ratio Monitoring of ${ }^{13} \mathrm{C}$ Enriched Butenolide

The isotope ratio monitoring was performed with a Finnigan 4500 mass spectrometer after gas chromotography, with a standard DB5 capillary column. All organic compounds in the sample are converted to

$\mathrm{CO}_{2}$ and $\mathrm{H}_{2} \mathrm{O}$, by combustion in a combustion furnace with . a cuo catalyst.. The amount of ${ }^{13} \mathrm{C}$ incorporation can then be measured by the ratio of mass $45^{-}\left(13 \mathrm{CO}_{2}\right)$ over masso $4\left({ }^{12} \mathrm{CO}_{2}\right)$.

This isotope ratio monitoring teghnique was applied to the purified ${ }^{3} \mathrm{C}$ enriched butenolide and the incorporation of ${ }^{13} \mathrm{C}$ was determined to be $9.88^{\circ}$ as opposed to the 1.18 natural apundance ${ }^{13} \mathrm{C} /{ }^{12} \mathrm{C}$ ratio.

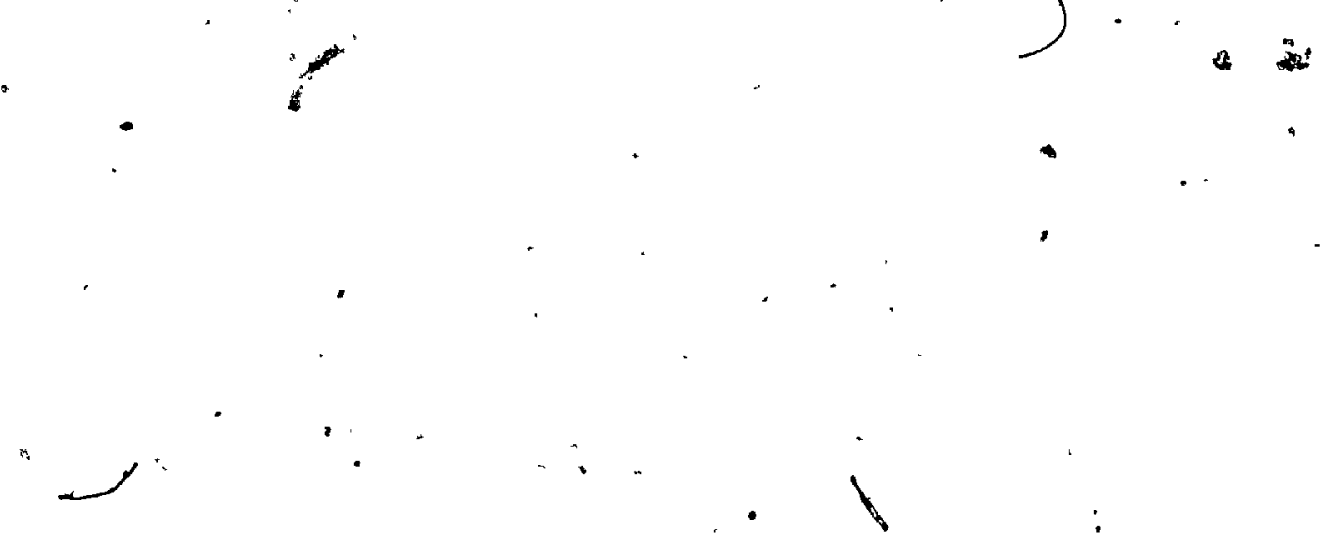


